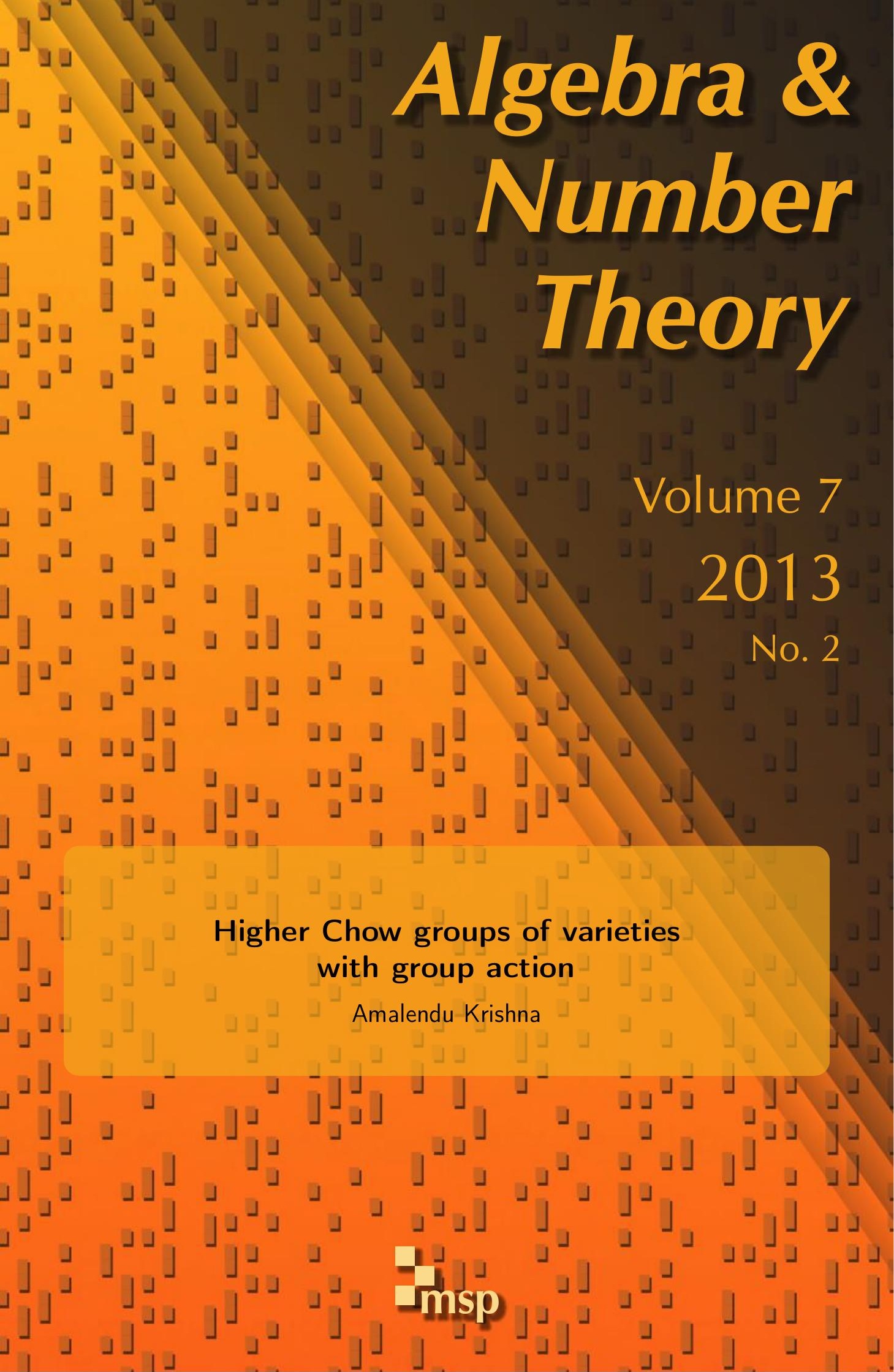




\title{
Higher Chow groups of varieties with group action
}

\author{
Amalendu Krishna
}

\begin{abstract}
We give explicit descriptions of the higher Chow groups of toric bundles and flag bundles over schemes. We derive several consequences of these descriptions for the equivariant and ordinary higher Chow groups of schemes with group action.

We prove a decomposition theorem for the equivariant higher Chow groups of a smooth scheme with action of a diagonalizable group. This theorem is applied to compute the equivariant and ordinary higher Chow groups of smooth toric varieties. The results of this paper play fundamental roles in the proof of the Riemann-Roch theorems for equivariant higher $K$-theory.
\end{abstract}

\section{Introduction}

A scheme in this paper will mean a separated and reduced scheme of finite type over a perfect field $k$, which admits an ample line bundle. This base field $k$ will be fixed throughout this paper. A linear algebraic group $G$ over $k$ will mean a smooth and affine group scheme over $k$. By a closed subgroup $H$ of an algebraic group $G$, we shall mean a morphism $H \rightarrow G$ of algebraic groups over $k$ that is a closed immersion of $k$-schemes. In particular, a closed subgroup of a linear algebraic group will be of the same type and hence smooth. Recall from [Borel 1991, Proposition 1.10] that a linear algebraic group over $k$ is a closed subgroup of a general linear group, defined over $k$.

Recall that an action of a linear algebraic group $G$ on a $k$-scheme $X$ is said to be linear if $X$ admits a $G$-equivariant ample line bundle, a condition that is always satisfied if $X$ is normal (see [Sumihiro 1975, Theorem 2.5] for $G$ connected and [Thomason 1988, 5.7] for $G$ general). All $G$-actions in this paper will be assumed to be linear. Let $\mathscr{V}_{k}$ denote the category of quasiprojective $k$-schemes and let $\mathscr{V}_{k}^{S}$ denote the full subcategory of smooth $k$-schemes. We shall denote the category of quasiprojective $G$-schemes with $G$-equivariant maps by $\mathscr{V}_{G}$, and the full subcategory of smooth $G$-schemes will be denoted by ${ }^{S}{ }_{G}^{S}$.

MSC2010: primary 14C40, 14C35; secondary 14C25.

Keywords: algebraic cycles, group action. 
The $G$-equivariant higher Chow groups $\mathrm{CH}_{*}^{G}(X, i)$ of $X \in \mathscr{V}_{G}$ were defined by Edidin and Graham [1998] in terms of the ordinary higher Chow groups (motivic Borel-Moore homology) of the quotient space $X \times{ }^{G} U$. Here, $U$ is a $G$-invariant open subscheme of a finite-dimensional representation $V$ of $G$ such that it acts freely on $U$ and $V \backslash U$ is of sufficiently high codimension. This definition of Edidin and Graham is based on an earlier construction of Totaro [1999], who invented the idea above to define the Chow groups of the classifying spaces of linear algebraic groups.

In this paper, we develop further the Edidin-Graham theory of equivariant higher Chow groups and establish many important properties of this theory. We also prove some decomposition theorems for the equivariant higher Chow groups of smooth schemes with torus action. These results turn out to have many applications.

Brion [1997] proved many results about the equivariant Chow groups of the form $\mathrm{CH}_{*}^{G}(X, 0)$. Many of the structural results in this paper can be described as the generalization of the results of [Brion 1997] to the case of equivariant higher Chow groups. In Section 2, we recall the definition of equivariant higher Chow groups from [Edidin and Graham 1998] and prove its basic properties, which are all well known for the ordinary higher Chow groups; see [Bloch 1986]. As a consequence, one finds that the equivariant higher Chow groups form a Borel-Moore oriented bigraded homology theory in the category of schemes with the action of a given linear algebraic group. Other important results about these groups such as the Morita isomorphism are proven in Section 3. We also prove a structure theorem (see Theorem 3.5) for the equivariant higher Chow groups of schemes with action of tori.

Section 4 contains the proof of the self-intersection formula for the higher Chow groups. This formula plays a very important role in the proofs of the main results of this paper. In Section 5, we construct Demazure operators on equivariant higher Chow groups and give some consequences of these operators.

In Section 6, we prove the Leray-Hirsch theorem for the higher Chow groups. As a consequence of this theorem, we compute the higher Chow groups of toric bundles in Section 7. In Section 8, we turn to the description of the higher Chow groups of principal bundles and flag bundles over schemes. We give several applications of these descriptions in the study of equivariant higher Chow groups.

In Sections 9 and 10, we prove a decomposition theorem (see Theorem 10.3) for the equivariant higher Chow groups of smooth schemes with action of a diagonalizable group $G$. This result describes the equivariant higher Chow group of a $G$-scheme in terms of the equivariant higher Chow groups of the loci where the stabilizers have a fixed dimension. This result is an analogue of a similar result of Vezzosi and Vistoli [2003] in equivariant $K$-theory and has many important 
applications in the study of equivariant and ordinary higher Chow groups of smooth schemes.

Theorem 10.3 is the basic step in the proof of the equivariant Riemann-Roch theorem in [Krishna 2009b]. This theorem presents an explicit relation between the equivariant $K$-theory and the equivariant higher Chow groups. Like in the ordinary case, this Riemann-Roch is a fundamental result in equivariant algebraic geometry. This theorem was in fact one of the main motivations for the author to work on this paper. We expect Theorem 10.3 to have many more applications in the computation of equivariant and ordinary higher Chow groups. In Section 11, we apply this theorem to compute the equivariant and ordinary higher Chow groups of smooth toric varieties. We shall follow the following convention while studying the equivariant and ordinary higher Chow groups with the rational coefficients.

Convention. In this paper, all the results and statements up to Section 7 do not make any assumption on the coefficient ring of the higher Chow groups. On the other hand, all the results and statements from Section 8 onwards assume rational coefficients. In order to simplify the notation, the following convention will be followed.

From Section 8 onwards, an abelian group $A$ will actually mean its extension $A \otimes_{\mathbb{Z}} \mathbb{Q}$. In particular, all higher Chow groups and other cohomology groups will be considered with the rational coefficients. For $\mathbb{Q}$-vector spaces $A$ and $B$, the tensor product $A \otimes_{\mathbb{Q}} B$ will be simply written as $A \otimes B$. We shall however, indicate the appropriate coefficients in the statements of the all results.

\section{Equivariant higher Chow groups}

In this section, we recall the definition of the equivariant higher Chow groups from [Edidin and Graham 1998] and review their main functorial properties. It turns out in particular that the equivariant higher Chow groups have all the properties of an oriented bigraded Borel-Moore homology theory.

Let $G$ be a linear algebraic group and let $X$ be a scheme over $k$ with a $G$-action. We shall denote the dimension of the underlying group $G$ usually by the letter $g$. All representations of $G$ in this paper will be finite-dimensional. The definition of equivariant higher Chow groups of $X$ needs one to consider certain kind of mixed spaces which in general may not be schemes even if the original spaces are schemes. The following well-known (see [Edidin and Graham 1998, Proposition 23]) lemma shows that this problem does not occur in our context and all the mixed spaces in this paper are schemes with ample line bundles.

Lemma 2.1. Let $H$ be a linear algebraic group acting freely and linearly on a $k$-scheme $U$ such that the quotient $U / H$ exists as a quasiprojective scheme. Let $X$ be a $k$-scheme with a linear action of $H$. Then the mixed quotient $X \times{ }^{H} U$ exists for 
the diagonal action of $H$ on $X \times U$ and is quasiprojective. Moreover, this quotient is smooth if both $U$ and $X$ are so. In particular, if $H$ is a closed subgroup of a linear algebraic group $G$ and $X$ is a $k$-scheme with a linear action of $H$, then the quotient $G \times{ }^{H} X$ is a quasiprojective scheme.

Proof. It is already shown in [Edidin and Graham 1998, Proposition 23] using [Mumford et al. 1994, Proposition 7.1] that the quotient $X \times{ }^{H} U$ is a scheme. Moreover, as $U / H$ is quasiprojective, [Mumford et al. 1994, Proposition 7.1] in fact shows that $X \times{ }^{H} U$ is also quasiprojective. The similar conclusion about $G \times{ }^{H} X$ follows from the first case by taking $U=G$ and by observing that $G / H$ is a smooth quasiprojective scheme; see [Borel 1991, Theorem 6.8]. The assertion about the smoothness is clear since $X \times U \rightarrow X \times{ }^{H} U$ is a principal $H$-bundle.

2a. Good pairs and equivariant higher Chow groups. For any integer $j \geq 0$, let $V$ be an $l$-dimensional representation of $G$ and let $U$ be a $G$-invariant open subset of $V$ such that the codimension of the complement $V \backslash U$ in $V$ is sufficiently larger than $j$, and $G$ acts freely on $U$ such that the quotient $U / G$ is a quasiprojective scheme. Such a pair $(V, U)$ will be called a good pair for the $G$-action corresponding to $j$. It is easy to see that a good pair always exists; see [Edidin and Graham 1998, Lemma 9].

For an equidimensional $G$-scheme $X$, let $X_{G}$ denote the quotient $X \times{ }^{G} U$ of the product $X \times U$ by the diagonal action of $G$, which is free. We define the equivariant higher Chow group $\mathrm{CH}_{G}^{j}(X, i)$ as the homology group $H_{i}\left(\mathscr{L}^{j}\left(X_{G}, \bullet\right)\right)$, where $\mathscr{L}^{j}\left(X_{G}, \bullet\right)$ is the Bloch cycle complex of the scheme $X_{G}$. It is known [Edidin and Graham 1998, Section 2] that this definition of $\mathrm{CH}_{G}^{j}(X, i)$ is independent of the choice of a good pair $(V, U)$ for the $G$-action up to unique isomorphisms. One should also observe that $\mathrm{CH}_{G}^{j}(X, i)$ may be nonzero for infinitely many values of $j$, a crucial change from the case of nonequivariant (ordinary) higher Chow groups.

If $X$ is of dimension $d$, which is not necessarily equidimensional, one defines the equivariant higher Chow groups as

$$
\mathrm{CH}_{j}^{G}(X, i):=H_{i}\left(\mathscr{E}_{j+l-g}\left(X_{G}, \bullet\right)\right),
$$

where $(V, U)$ is an $l$-dimensional good pair for the $G$-action corresponding to $d-j$, and $\mathscr{E}_{p}\left(X_{G}, \bullet\right)$ is the homological cycle complex of Bloch such that $\mathscr{E}_{p}\left(X_{G}, i\right)$ is the group of admissible algebraic cycles on $X_{G} \times \Delta^{i}$ of dimension $p+i$. We write

$$
\mathrm{CH}_{*}^{G}(X, i)=\bigoplus_{-\infty<j \leq d} \mathrm{CH}_{j}^{G}(X, i) \quad \text { and } \quad \mathrm{CH}_{*}^{G}(X)=\bigoplus_{i \geq 0} \mathrm{CH}_{*}^{G}(X, i) .
$$

It is easy to see that $\mathrm{CH}_{j}^{G}(X, i)=\mathrm{CH}_{G}^{d-j}(X, i)$ if $X$ is equidimensional of dimension $d$. For most of this paper, we shall use the cohomological indexing for the equivariant 
higher Chow groups while dealing with smooth schemes. In particular, $\mathrm{CH}_{G}^{*}(X)$ will denote the sum $\bigoplus_{i \geq 0} \mathrm{CH}_{G}^{*}(X, i)$.

For a commutative ring $R$, the equivariant higher Chow groups $\mathrm{CH}_{G}^{j}(X, i ; R)$ are defined as the homology groups of the complex $\mathscr{L}^{j}\left(X_{G}, \bullet\right) \otimes_{\mathbb{Z}} R$. The symbol $\mathrm{CH}_{G}^{*}(X ; R)$ will denote the direct sum of all $\mathrm{CH}_{G}^{j}(X, i ; R)$. We shall denote the rings $\mathrm{CH}_{G}^{*}(k, 0)$ and $\mathrm{CH}_{G}^{*}(k, 0 ; R)$ by $S(G)$ and $S(G ; R)$, respectively.

2b. Equivariant operational Chow groups. For $X \in \mathscr{V}_{G}$, we define

$$
\mathrm{OPCH}_{G}^{j}(X, i)=\underset{\lim }{\longrightarrow} \mathrm{CH}_{G}^{j}(Y, i)
$$

where the limit is taken over the category of arrows $X \rightarrow Y$ in $\mathscr{V}_{G}$ with $Y \in \mathscr{V}_{G}^{S}$. Notice that the natural map $\mathrm{OPCH}_{G}^{j}(X, i) \rightarrow \mathrm{CH}_{G}^{j}(X, i)$ is an isomorphism if $X$ is smooth. We shall write the sum $\bigoplus_{i, j \geq 0} \operatorname{OPCH}_{G}^{j}(X, i)$ as $\mathrm{OPCH}_{G}^{*}(X)$.

It follows from [Bloch 1986, Proposition 5.5, Corollary 5.6] that $\mathrm{OPCH}_{G}^{*}(X)$ has a ring structure and $\mathrm{OPCH}_{G}^{*}(X, 0)$ is a subring of $\mathrm{OPCH}_{G}^{*}(X)$. Moreover, $X \mapsto \mathrm{OPCH}_{G}^{*}(X)$ is a contravariant functor on $\mathscr{V}_{G}$ which acts on the higher Chow groups of $X$. In particular, $\mathrm{OPCH}_{G}^{1}(X, 0) \stackrel{\sim}{\longrightarrow} \operatorname{Pic}_{G}(X)$ acts on $\mathrm{CH}_{*}^{G}(X, i)$. This action is same as the action of the Chern classes of equivariant line bundles on the homology theory $\mathrm{CH}_{*}^{G}(X, i)$.

2c. Main properties of equivariant higher Chow groups. The following result summarizes most of the essential properties of the equivariant higher Chow groups that will be used in this paper.

Proposition 2.2. The equivariant higher Chow groups as defined above satisfy the following properties.

(1) Functoriality: Covariance for proper maps, contravariance for flat maps and their compatibility. That is, for a fiber diagram

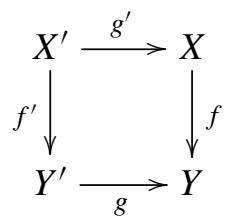

in $\mathscr{V}_{G}$ with $f$ proper and $g$ flat, one has

$$
g^{*} \circ f_{*}=f^{\prime} \circ g^{\prime *}: \mathrm{CH}_{*}^{G}(X, i) \rightarrow \mathrm{CH}_{*}^{G}\left(Y^{\prime}, i\right) .
$$

Moreover, if $f: X \rightarrow Y$ is a morphism in $\mathscr{V}_{G}$ with $Y$ in $\mathscr{V}_{G}^{S}$, then there is a pull-back map $f^{*}: \mathrm{CH}_{*}^{G}(Y, i) \rightarrow \mathrm{CH}_{*}^{G}(X, i)$.

(2) Homotopy: If $f: X \rightarrow Y$ is an equivariant vector bundle, then

$$
f^{*}: \mathrm{CH}_{*}^{G}(Y, i) \stackrel{\sim}{\longrightarrow} \mathrm{CH}_{*}^{G}(X, i) .
$$


(3) Exterior product: There is a natural product map

$$
\mathrm{CH}_{*}^{G}(X, i) \otimes \mathrm{CH}_{*}^{G}\left(Y, i^{\prime}\right) \rightarrow \mathrm{CH}_{*}^{G}\left(X \times Y, i+i^{\prime}\right) .
$$

Moreover, if $f: X \rightarrow Y$ is such that $Y \in \mathscr{V}_{G}^{S}$, then there is a pull-back via the graph map $\Gamma_{f}: X \rightarrow X \times Y$, which makes $\mathrm{CH}_{G}^{*}(Y)$ a bigraded ring and $\mathrm{CH}_{*}^{G}(X)$ a module over this ring. In particular, $\mathrm{CH}_{*}^{G}(X, i)$ an $S(G)$-module for $X \in \mathscr{V}_{G}$ and $i \geq 0$.

(4) Localization: If $Y \subset X$ is a $G$-invariant closed subscheme with complement $U$, then there is a long exact localization sequence of $S(G)$-modules

$\cdots \rightarrow \mathrm{CH}_{*}^{G}(Y, i) \rightarrow \mathrm{CH}_{*}^{G}(X, i) \rightarrow \mathrm{CH}_{*}^{G}(U, i) \rightarrow \mathrm{CH}_{*}^{G}(Y, i-1) \rightarrow \cdots$.

This sequence is compatible with the push-forward and flat pull-back maps of higher Chow groups.

(5) Chern classes: For any G-equivariant vector bundle of rank $r$, there are equivariant Chern classes $c_{l}^{G}(E): \mathrm{CH}_{j}^{G}(X, i) \rightarrow \mathrm{CH}_{j-l}^{G}(X, i)$ for $0 \leq l \leq r$, having the same functoriality properties as in the nonequivariant case and $c_{0}^{G}(E)=1$.

(6) Projection formula: For a proper map $f: X \rightarrow Y$ in $\mathscr{V}_{G}$ and for $x \in \mathrm{CH}_{*}^{G}(X)$, $y \in \operatorname{OPCH}_{G}^{*}(Y)$, one has $f_{*}\left(f^{*}(y) \cdot x\right)=y \cdot f_{*}(x)$. Here, the action of $\mathrm{OPCH}_{G}^{*}(Y)$ on $\mathrm{CH}_{*}^{G}(X)$ is given by (3) above.

(7) Free action: If $G$ acts freely on $X$ with quotient $Y$, then there is a canonical isomorphism $\mathrm{CH}_{*}^{G}(X, i) \stackrel{\sim}{\longrightarrow} \mathrm{CH}_{*}(Y, i)$.

Proof. Since the equivariant higher Chow groups of $X$ are defined in terms of the higher Chow groups of $X_{G}$, the proposition (except possibly the last property) can be easily deduced from the similar results for the nonequivariant higher Chow groups as in [Bloch 1986] and the techniques of [Edidin and Graham 1998]. We therefore skip the proof. To see that the maps in the localization sequence are $S(G)$-linear, it suffices to know that for a good pair $(V, U)$, the long exact sequence

$$
\cdots \rightarrow \mathrm{CH}_{*}\left(Y_{G}, i\right) \rightarrow \mathrm{CH}_{*}\left(X_{G}, i\right) \rightarrow \mathrm{CH}_{*}\left(U_{G}, i\right) \rightarrow \mathrm{CH}_{*}\left(Y_{G}, i-1\right) \rightarrow \cdots
$$

is a sequence of $\mathrm{CH}_{*}(U / G)$-modules. But this is a well-known fact as $U / G$ is smooth and the above is a sequence of higher Chow groups of schemes over it; see [Bloch 1986, Exercise 5.8(ii)].

To prove (6), we need to show that if $Y \stackrel{g}{\rightarrow} Z$ is a $G$-equivariant map with $Z \in \mathcal{V}_{G}^{S}$, then the $\operatorname{map} \mathrm{CH}_{*}^{G}(X) \stackrel{f_{*}}{\rightarrow} \mathrm{CH}_{*}^{G}(Y)$ is $\mathrm{CH}_{G}^{*}(Z)$-linear. Since the push-forward and the pull-back maps of equivariant Chow groups are nothing but the maps of ordinary higher Chow groups of suitable mixed quotients, it suffices to prove the statement above for the push-forward map of the higher Chow groups corresponding to the 
maps of mixed quotients $X_{G} \rightarrow Y_{G} \rightarrow Z_{G}$. Since $Z_{G}$ is smooth, this nonequivariant version is well-known [Bloch 1986, §5.5, Exercise 5.8].

For the last property, fix $j \leq d$ and choose a good pair $(V, U)$ of dimension $l$ for the $G$-action corresponding to $d-j \geq 0$. Since $G$ acts freely on $X$, it acts likewise also on $X \times V$ with quotient, say $X_{V}$. Then $X_{G}$ is an open subset of $X_{V}$ and $X_{V} \rightarrow Y$ is a vector bundle, which implies that the map $\mathrm{CH}_{j}(Y, i) \rightarrow \mathrm{CH}_{j+l}\left(X_{V}, i\right)$ is an isomorphism by the homotopy invariance. On the other hand, the restriction map $\mathrm{CH}_{j+l}\left(X_{V}, i\right) \rightarrow \mathrm{CH}_{j+l}\left(X_{G}, i\right)=\mathrm{CH}_{j-g}^{G}(X, i)$ is an isomorphism by the property (4) as $d-j$ is sufficiently small.

Remark 2.3. The reader should be warned that the various isomorphisms between the (equivariant) higher Chow groups in the proposition above are true only up to some obvious shift in the dimension of cycles, which we have chosen not to write.

We next recall from [Edidin and Graham 1998] that the Chern classes $c_{l}^{G}(E)$ of an equivariant vector bundle $E$, as described in Proposition 2.2 above, live in the operational Chow groups $\mathrm{OPCH}^{l}\left(X_{G}\right)$. If $X$ is in $\mathscr{V}_{G}^{S}$ however, this operational Chow group is isomorphic to the equivariant Chow group $\mathrm{CH}_{G}^{l}(X, 0)$ and the action of $c_{l}^{G}(E)$ on $\mathrm{CH}_{G}^{*}(X)$ then coincides with the intersection product in the ring $\mathrm{CH}_{G}^{*}(X)$.

Finally, we recall from [ibid.] that if $H \subset G$ is a closed subgroup and if $(V, U)$ is a good pair, then for $X \in \mathscr{V}_{G}$, the natural map of quotients $X \times{ }^{H} U \rightarrow X \times{ }^{G} U$ is an étale locally trivial $G / H$-fibration and hence there is a natural restriction map

$$
r_{H, X}^{G}: \mathrm{CH}_{*}^{G}(X, i) \rightarrow \mathrm{CH}_{*}^{H}(X, i) .
$$

Taking $H=\{1\}$, one obtains the forgetful map

$$
r_{X}^{G}: \mathrm{CH}_{*}^{G}(X, i) \rightarrow \mathrm{CH}_{*}(X, i) .
$$

Moreover, as $r_{H, X}^{G}$ is the pull-back under a flat (in fact, a smooth) map, it commutes (see Proposition 2.2) with the pull-back for any flat map, and with the push-forward for any proper map in $\mathscr{V}_{G}$. We remark here that although the definition of $r_{H, X}^{G}$ uses a good pair $(V, U)$ for any given $j \leq \operatorname{dim}(X)$, it is easy to check from the homotopy invariance that $r_{H, X}^{G}$ is independent of the choice of the good pair $(V, U)$.

\section{Morita isomorphisms}

In this section, we prove some Morita-type isomorphisms that address the question of comparison between the equivariant higher Chow groups for the action of two different algebraic groups. We also prove a structure theorem for these equivariant higher Chow groups under the trivial action of split tori. These results are analogues of the similar results of Thomason in equivariant $K$-theory; see [Thomason 1986, Lemma 5.6; 1988, Section 1]. 
Proposition 3.1 (Morita isomorphism). Let $H$ be a normal subgroup of a linear algebraic group $G$ and let $F=G / H$. Let $f: X \rightarrow Y$ be a $G$-equivariant morphism of $G$-varieties that is an $H$-torsor for the restricted action. Then the map induced on the equivariant higher Chow groups

$$
\mathrm{CH}_{*}^{F}(Y, i) \stackrel{f^{*}}{\rightarrow} \mathrm{CH}_{*}^{G}(X, i) .
$$

is an isomorphism.

Proof. We first observe from [Springer 1998, Corollary 12.2.2] that $F$ is also a linear algebraic group over the given ground field $k$. Now, since $f$ is an $H$-torsor, it is clear that $G$ acts on $Y$ via $F$. Fix $j \leq \operatorname{dim}(X)$ and choose a good pair $(V, U)$ of dimension $l$ for the $F$-action corresponding to $\operatorname{dim}(Y)-j$. Then $V$ is also a representation of $G$ in which $U$ is $G$-invariant. In particular, $G$ acts on $X \times U$ via the diagonal action, which is easily seen to be free since $H$ acts freely on $X$ and $F$ acts freely on $U$. By the same reason, we see that $X \times U \rightarrow Y \times U$, which is a principal $H$-bundle, is $G$-equivariant. This in turn implies that the map $(X \times U) / G \rightarrow Y_{F}$ is an isomorphism and hence we get

$$
\mathrm{CH}_{j}^{F}(Y, i) \cong \mathrm{CH}_{j+l-g+h}\left(Y_{F}, i\right) \underset{f^{*}}{\stackrel{\sim}{\longrightarrow}} \mathrm{CH}_{j+l-g+h}\left(X \times{ }^{G} U, i\right),
$$

where $\operatorname{dim}(H)=h$. On the other hand, we have

$\mathrm{CH}_{j+h}^{G}(X, i) \cong \mathrm{CH}_{j+h+l}^{G}(X \times V, i) \cong \mathrm{CH}_{j+h+l}^{G}(X \times U, i) \cong \mathrm{CH}_{j+h+l-g}\left(X \times{ }^{G} U, i\right)$,

where the first isomorphism is due to the homotopy invariance, the second follows from the localization property (see Proposition 2.2(4)) as $j$ is sufficiently small, and the third isomorphism follows from Proposition 2.2(7). The proof of the proposition now follows by combining this with (3-1).

Corollary 3.2 (see [Edidin and Graham 2000]). Let $H \subset G$ be a closed subgroup and let $X \in \mathscr{V}_{H}$. Then for any $i \geq 0$, there is a natural isomorphism

$$
\mathrm{CH}_{*}^{G}\left(G \times{ }^{H} X, i\right) \stackrel{\sim}{\longrightarrow} \mathrm{CH}_{*}^{H}(X, i) .
$$

Proof. Define an action of $H \times G$ on $G \times X$ by

$$
(h, g) \cdot\left(g^{\prime}, x\right)=\left(g g^{\prime} h^{-1}, h x\right)
$$

and an action of $H \times G$ on $X$ by $(h, g) \cdot x=h x$. Then the projection map $G \times X \stackrel{p}{\rightarrow} X$ is $(H \times G)$-equivariant and is a $G$-torsor. Hence by Proposition 3.1, the natural map

$$
\mathrm{CH}_{*}^{H}(X, i) \stackrel{p^{*}}{\rightarrow} \mathrm{CH}_{*}^{H \times G}(G \times X, i)
$$


is an isomorphism. On the other hand, the projection map $G \times X \rightarrow G \times{ }^{H} X$ is $(H \times G)$-equivariant and is an $H$-torsor. Hence we get an isomorphism

$$
\mathrm{CH}_{*}^{G}\left(G \times{ }^{H} X, i\right) \stackrel{\sim}{\longrightarrow} \mathrm{CH}_{*}^{H \times G}(G \times X, i) .
$$

The corollary follows by combining these two isomorphisms.

Proposition 3.3. Let $G$ be a connected reductive group over $k$. Let $B$ be a Borel subgroup of $G$ containing a maximal torus $T$ over $k$. Then for any $i \geq 0$, the restriction map

$$
\mathrm{CH}_{*}^{B}(X, i) \stackrel{r_{T, X}^{B}}{\longrightarrow} \mathrm{CH}_{*}^{T}(X, i)
$$

is an isomorphism for any $X \in \mathscr{V}_{B}$.

Proof. By Corollary 3.2, we only need to show that

$$
\mathrm{CH}_{*}^{B}(X, i) \stackrel{\sim}{\longrightarrow} \mathrm{CH}_{*}^{B}\left(B \times{ }^{T} X, i\right) .
$$

By [M. Demazure 1970, XXII, 5.9.5], there exists a characteristic filtration

$$
B^{u}=U_{0} \supseteq U_{1} \supseteq \cdots \supseteq U_{n}=\{1\}
$$

of the unipotent radical $B^{u}$ of $B$ such that $U_{j-1} / U_{j}$ is a vector group, each $U_{j}$ is normal in $B$ and $T U_{j}=T \ltimes U_{j}$. Moreover, this filtration also implies that for each $j$, the natural map $B / T U_{j} \rightarrow B / T U_{j-1}$ is a torsor under the vector bundle $U_{j-1} / U_{j} \times B / T U_{j-1}$ on $B / T U_{j-1}$. Hence, the homotopy invariance gives an isomorphism

$$
\mathrm{CH}_{*}^{B}\left(B / T U_{j-1} \times X, i\right) \stackrel{\sim}{\longrightarrow} \mathrm{CH}_{*}^{B}\left(B / T U_{j} \times X, i\right) .
$$

Composing these isomorphisms successively for $j=1, \ldots, n$, we get

$$
\mathrm{CH}_{*}^{B}(X, i) \stackrel{\sim}{\longrightarrow} \mathrm{CH}_{*}^{B}(B / T \times X, i) .
$$

The canonical isomorphism of $B$-varieties $B \times{ }^{T} X \cong B / T \times X$ (see Corollary 3.2) now proves (3-5) and hence (3-4).

Recall that a linear algebraic group $G$ over $k$ of dimension $g$ is diagonalizable if $G_{\bar{k}} \cong H \times\left(\mathbb{G}_{m}\right)^{g}$, where $H$ is a finite abelian group. The group $G$ is called split diagonalizable, if such an isomorphism is defined over $k$. A connected reductive group $G$ over $k$ is said to be split if it contains a split maximal torus $T$ over $k$ such that $G$ is given by a root system relative to $T$. Every connected and reductive group containing a split maximal torus is split; see [M. Demazure 1970, Chapter XXII, Proposition 2.1].

Recall from [Springer 1998, Lemma 14.1.1] that every solvable group $G$ over $k$ has a filtration $\{e\}=G_{0} \subseteq G_{1} \subseteq \cdots \subseteq G_{n}=G$ by closed normal $k$-subgroups such that each quotient group $G_{j} / G_{j-1}$ is either diagonalizable or an elementary 
unipotent group; see [Springer 1998, §3.4]. The group $G$ is called split over $k$ if each $G_{j} / G_{j-1}$ is either split diagonalizable or $\mathbb{G}_{a}$. It is known [Springer 1998, Corollary 14.3.10] that every unipotent group over a perfect field is split.

Proposition 3.4. Let $H$ be a possibly nonreductive group over $k$. Assume that $H$ has a Levi decomposition $H=L \ltimes H^{u}$ such that $H^{u}$ is split over $k$ (for example, if $k$ is of characteristic zero). Then for each $i \geq 0$, the map

$$
\mathrm{CH}_{*}^{H}(X, i) \stackrel{r_{L, X}^{H}}{\longrightarrow} \mathrm{CH}_{*}^{L}(X, i)
$$

is an isomorphism.

Proof. Since the unipotent radical of $H$ is split over $k$, the proof is exactly same as in the proof of (3-4), where we just have to replace $B$ and $T$ by $H$ and $L$, respectively.

3a. A structure theorem for $\mathbf{C H}_{*}^{T}(X)$. We end this section with the following structure theorem for the equivariant higher Chow groups of a scheme with the action of a split diagonalizable group on which certain subgroup acts trivially. This theorem is the initial step in the proof of its far reaching generalization in Theorem 10.3.

Theorem 3.5. Let $T$ be a split diagonalizable group and let $X \in \mathscr{V}_{T}$. Let $H$ be a connected closed subgroup of $T$ that acts trivially on $X$. Then there is a natural isomorphism

$$
\mathrm{CH}_{*}^{T / H}(X) \otimes_{\mathbb{Z}} S(H) \stackrel{i_{H, X}^{T}}{\longrightarrow} \mathrm{CH}_{*}^{T}(X) .
$$

This is a bigraded ring isomorphism if $X$ is smooth.

Proof. Put $T^{\prime}=T / H$. Since $H$ is a split torus, we can choose a decomposition (not necessarily canonical) $T=H \times T^{\prime}$. Fix an integer $j \leq \operatorname{dim}(X)$ and let $(V, U)$ and $\left(V^{\prime}, U^{\prime}\right)$ be good pairs for the actions of $H$ and $T^{\prime}$, respectively, corresponding to $\operatorname{dim}(X)-j$ as in [Edidin and Graham 1998, Example 3.1]. Thus $U$ is a product of punctured affine spaces and $U / H=\left(\mathbb{P}^{n}\right)^{r}$ for some $n \gg 0$, where $r=\operatorname{rank}(H)$. Then $\left(V_{T}, U_{T}\right)$, with $V_{T}=V \times V^{\prime}$ and $U_{T}=U \times U^{\prime}$, is a good pair for the action of $T$. We now have

$$
X_{T}=\left(X \times U \times U^{\prime}\right) /\left(H \times T^{\prime}\right)=\left(X \times U^{\prime}\right) \times^{T^{\prime}} U / H=X_{T^{\prime}} \times\left(\mathbb{P}^{n}\right)^{r},
$$

where the second equality holds since $H$ acts trivially on $X \times U^{\prime}$ and the third equality holds because $T^{\prime}$ acts trivially on $U$. It follows from the projective bundle formula (see also Lemma 6.2) for the ordinary higher Chow groups that the map

$$
\mathrm{CH}_{*}\left(X_{T^{\prime}}\right) \otimes_{\mathbb{Z}} \mathrm{CH}_{*}\left(\left(\mathbb{P}^{n}\right)^{r}, 0\right) \rightarrow \mathrm{CH}_{*}\left(X_{T}\right)
$$


is an isomorphism. We conclude the proof by noting that $\mathrm{CH}_{p}\left(X_{T^{\prime}}\right) \cong \mathrm{CH}_{p}^{T^{\prime}}(X)$ and $\mathrm{CH}_{p}\left(X_{T}\right) \cong \mathrm{CH}_{p}^{T}(X)$ for all $p \leq j$. If $X$ is smooth, the assertion about the ring isomorphism of $i_{H, X}^{T}$ now follows because (3-7) is known to be a bigraded ring isomorphism in that case.

\section{Self-intersection and projection formulas}

Our aim in this section is to prove the following two results for the ordinary and equivariant higher Chow groups. The first result is the self-intersection formula for the higher Chow groups of smooth schemes. The analogue of this formula for the higher $K$-theory was proven by Thomason [1993, Theorem 3.1]. Surprisingly, this formula for the higher Chow groups has remained unnoticed. Its equivariant version will play a very crucial role in the decomposition Theorem 10.3 for the equivariant higher Chow groups of smooth schemes with an action of a diagonalizable group.

The second result of this section is a version of projection formula for the higher Chow groups of singular schemes. Such a formula for the smooth schemes was proven by Bloch [1986]. We shall need this version of the projection formula in our construction of Demazure operators on the equivariant higher Chow groups.

4a. Self-intersection formula. We shall use the method of deformation to the normal cone as the main technical tool to prove the self-intersection formula. Since this technique will be used several times in this paper, we briefly recall the construction from [Fulton 1984, Chapter 5] for our, as well as reader's, convenience. Let $X$ be a smooth scheme over $k$ and let $f: Y \hookrightarrow X$ be a smooth closed subscheme of codimension $d \geq 1$. Let $\widetilde{M}$ be the blow-up of $X \times \mathbb{P}^{1}$ along $Y \times \infty$. Then $B l_{Y}(X)$ is a closed subscheme of $\widetilde{M}$ and one denotes its complement by $M$. There is a natural map $\pi: M \rightarrow \mathbb{P}^{1}$ such that $\pi^{-1}\left(\mathbb{A}^{1}\right) \cong X \times \mathbb{A}^{1}$ with $\pi$ the projection map and $\pi^{-1}(\infty) \cong X^{\prime}$, where $X^{\prime}$ is the total space of the normal bundle $N_{Y / X}$ of $Y$ in $X$. One also gets the following diagram, where all the squares and the triangles commute.

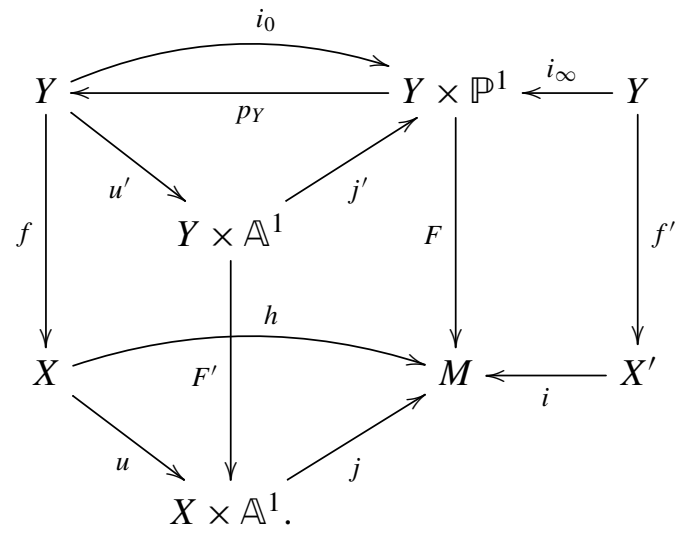


In this diagram, all the vertical arrows are the closed embeddings, $i_{0}$ and $i_{\infty}$ are the obvious inclusions of $Y$ in $Y \times \mathbb{P}^{1}$ along the specified points, $i$ and $j$ are inclusions of the inverse images of $\infty$ and $\mathbb{A}^{1}$, respectively, under the map $\pi, u$ and $f^{\prime}$ are zero section embeddings and $p_{Y}$ is the projection map. In particular, one has $p_{Y} \circ i_{0}=p_{Y} \circ i_{\infty}=\mathrm{id}_{Y}$.

In case $X$ is a $G$-scheme and $Y$ is $G$-invariant, then by letting $G$ act trivially on $\mathbb{P}^{1}$ and diagonally on $X \times \mathbb{P}^{1}$, one gets a natural action of $G$ on $M$, and all the spaces in the diagram above become $G$-spaces and all the morphisms become $G$-equivariant. This observation will be used later in this paper.

We shall need the following result about the higher Chow groups, which is an easy consequence of Bloch's moving lemma.

Lemma 4.1. Let

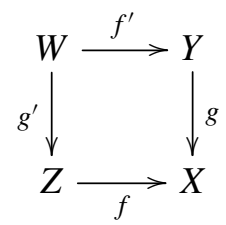

be a fiber diagram of closed immersions of schemes such that $X$ and $Y$ are smooth and $Y$ and $Z$ intersect properly in $X$. Then one has for each $i \geq 0$,

$$
f^{*} \circ g_{*}=g_{*}^{\prime} \circ f^{\prime *}: \mathrm{CH}_{*}(Y, i) \rightarrow \mathrm{CH}_{*}(Z, i) .
$$

Proof. Since $X$ and $Y$ are smooth, we can assume them to be equidimensional. Let

$$
\mathscr{E}_{Z W}^{p}(Y, \bullet) \stackrel{i_{Y}}{\hookrightarrow} \mathscr{E}^{p}(Y, \bullet)
$$

be the subcomplex that is generated by cycles on $Y \times \Delta^{\bullet}$ which intersect all faces of $Z \times \Delta^{\bullet}$ and $W \times \Delta^{\bullet}$ properly. Similarly, let

$$
\mathscr{L}_{Z}^{p}(X, \bullet) \stackrel{i_{X}}{\hookrightarrow} \mathscr{L}^{p}(X, \bullet)
$$

be the subcomplex generated by the cycles on $X \times \Delta^{\bullet}$ that intersect all faces of $Z \times \Delta^{\bullet}$ properly. Then $i_{X}$ and $i_{Y}$ are quasi-isomorphisms by the moving lemma; see [Krishna and Levine 2008, Theorem 1.10]. However, if $V \in \mathscr{L}_{Z W}^{p}(Y, \bullet)$ is an irreducible cycle in $Y \times \Delta^{n}$, then the conclusion of the lemma is checked easily.

Corollary 4.2. Let $G$ be a linear algebraic group and let

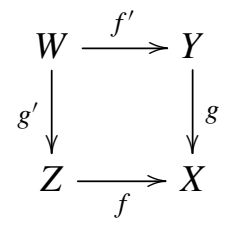


be a fiber diagram of closed immersions of smooth $G$-schemes such that $Y$ and $Z$ intersect properly in $X$. Then one has $f^{*} \circ g_{*}=g_{*}^{\prime} \circ f^{\prime *}: \mathrm{CH}_{G}^{*}(Y, i) \rightarrow \mathrm{CH}_{G}^{*}(Z, i)$. Proof. By choosing a good pair $(V, U)$ for the $G$-action and then considering the appropriate mixed quotients, we can reduce to proving the corollary for the nonequivariant higher Chow groups. But this is shown in Lemma 4.1.

Lemma 4.3. Consider the diagram (4-1) and let $y \in \mathrm{CH}^{*}(Y, m)$. Then there exists $z \in \mathrm{CH}^{*}(M, m)$ such that $f_{*}(y)=h^{*}(z)$ and $f^{\prime}{ }_{*}(y)=i^{*}(z)$.

Proof. Put $\tilde{y}=p_{Y}^{*}(y)$ and $z=F_{*}(\tilde{y})$. Then

$$
\begin{array}{rlrl}
f_{*}(y) & =f_{*}\left(\left(p_{Y} \circ i_{0}\right)^{*}(x)\right)=f_{*} \circ i_{0}^{*} \circ p_{Y}^{*}(y)=f_{*} \circ i_{0}^{*}(\tilde{y})=f_{*} \circ u^{\prime *} \circ j^{\prime *}(\tilde{y}) \\
& =u^{*} \circ F^{\prime}{ }_{*}\left(j^{\prime *}(\tilde{y})\right) & \text { (by Lemma 4.1) } \\
& =u^{*} \circ j^{*} \circ F_{*}(\tilde{y}) & \text { (since } j \text { is an open immersion) } \\
& =h^{*} \circ F_{*}(\tilde{y})=h^{*}(z) . &
\end{array}
$$

Similarly,

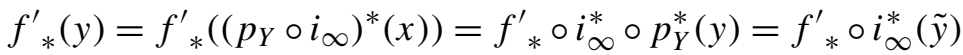

$$
\begin{aligned}
& =i^{*} \circ F_{*}(\tilde{y}) \\
& \text { (by Lemma 4.1) } \\
& =i^{*}(z) \text {. }
\end{aligned}
$$

Theorem 4.4 (self-intersection formula). Let $Y \stackrel{f}{\hookrightarrow} X$ be a closed immersion of smooth varieties of codimension $d \geq 0$, and let $N_{Y / X}$ be the normal bundle of $Y$ in $X$. Then for every $y \in \mathrm{CH}^{*}(Y, i)$, one has $f^{*} \circ f_{*}(y)=c_{d}\left(N_{Y / X}\right) \cdot y$.

Proof. There is nothing to prove when $d=0$ and so we assume $d \geq 1$. We first consider the case when $X \stackrel{p}{\rightarrow} Y$ is a vector bundle of rank $d$ and $f$ is the zero section embedding so that $p \circ f=\mathrm{id}_{Y}$. In that case, we have

$$
\begin{aligned}
f^{*} \circ f_{*}(y) & =f^{*} \circ f_{*}\left(f^{*} \circ p^{*}(y)\right)=f^{*}\left(f_{*}(1) \cdot p^{*}(y)\right) \quad \text { (by Proposition 2.2(6)) } \\
& =f^{*}\left(f_{*}(1)\right) \cdot\left(f^{*} \circ p^{*}(y)\right)=f^{*}\left(f_{*}(1)\right) \cdot y=c_{d}\left(N_{Y / X}\right) \cdot y,
\end{aligned}
$$

where the last equality follows from the self-intersection formula for Fulton's Chow groups; see [Fulton 1984, Corollary 6.3]. This proves the theorem in the case of zero section embedding.

Now let $Y \hookrightarrow X$ be as in the theorem. We consider the deformation to the normal cone diagram (4-1) and choose $z \in \mathrm{CH}^{*}(M, i)$ as in Lemma 4.3. Then we have

$$
\begin{array}{rlr}
f^{*} \circ f_{*}(y) & =f^{*} \circ h^{*}(z)=i_{0}{ }^{*} \circ F^{*}(z)=i_{\infty}^{*} \circ F^{*}(z)=f^{\prime *} \circ i^{*}(z) \\
& =f^{\prime *} \circ f^{\prime}{ }_{*}(y) & \text { (by Lemma 4.3) } \\
& =c_{d}\left(N_{Y / X^{\prime}}\right) \cdot y & \text { (by the case of vector bundle above) } \\
& =c_{d}\left(N_{Y / X}\right) \cdot y .
\end{array}
$$


This completes the proof of the theorem.

Corollary 4.5. Let $G$ be a linear algebraic group over $k$ and let $Y \stackrel{f}{\hookrightarrow} X$ be a closed immersion of codimension $d \geq 0$ in $\mathscr{V}_{G}^{S}$. Then for every $i \geq 0$ and $y \in \mathrm{CH}_{G}^{*}(Y, i)$, one has $f^{*} \circ f_{*}(y)=c_{d}^{G}\left(N_{Y / X}\right) \cdot y$.

Proof. There is nothing to prove if $d=0$ and so we assume $d \geq 1$. Fix $i, j \geq 0$ and choose a good pair $(V, U)$ for $n \gg j+d$. We can then identify $\mathrm{CH}_{G}^{p}(X, i)$ with $\mathrm{CH}^{p}\left(X_{G}, i\right)$ (and same for $Y$ ) for $p \leq n$. We can also identify $c_{d}^{G}(E)$ with $c_{d}\left(E_{G}\right)$ for any equivariant vector bundle $E$ on $Y$; see [Edidin and Graham 1998, Section 2.4]. Now, the proof of the corollary would follow straightaway from Theorem 4.4, once we show that $\left(N_{Y / X}\right)_{G}$ is the normal bundle of $Y_{G}$ in $X_{G}$. But this follows immediately from the elementary fact that if $G$ acts freely on a smooth scheme $Z$ and $W$ is a smooth closed and $G$-invariant subscheme of $Z$ with normal bundle $N$, then $G$ acts freely on $N$, and moreover, $N / G$ is the normal bundle of $W / G$ in $Z / G$. We leave the proof of this fact to the reader.

4b. A projection formula for singular schemes. Recall from Section $2 \mathrm{~b}$ (see also [Bloch 1986, Corollary 5.6]) that the operational Chow groups $X \mapsto \mathrm{OPCH}^{*}(X)$ form a ring-valued contravariant functor on $\mathscr{V}_{k}$ that acts on the higher Chow groups. The action of $\mathrm{OPCH}^{1}(X, 0) \stackrel{\sim}{\longrightarrow} \operatorname{Pic}(X)$ on $\mathrm{CH}_{*}(X, j)$ coincides with the action of the Chern classes of line bundles.

Proposition 4.6. Let $X \in \mathscr{V}_{k}$ and let $f: Y=\mathbb{P}(E) \rightarrow X$ be the projective bundle associated to a vector bundle $E$ of rank $n+1$ on $X$ and let

$$
\xi=c_{1}\left(\mathrm{O}_{Y}(1)\right) \in \mathrm{OPCH}^{1}(Y, 0)
$$

be the first Chern class of the relative tautological line bundle on $Y$. Then for any $x \in \mathrm{CH}_{*}(X, j)$, one has

$$
f_{*}\left(\xi^{i} \cdot f^{*}(x)\right)= \begin{cases}0 & \text { if } i<n, \\ x & \text { if } i=n .\end{cases}
$$

Proof. If $X$ is smooth, the proposition is an easy consequence of the projection formula [Bloch 1986, Exercise 5.8], as this formula implies that

$$
f_{*}\left(\xi^{i} \cdot f^{*}(x)\right)=f_{*}\left(\xi^{i}\right) \cdot x .
$$

Moreover, it follows from [Fulton 1984, Proposition 3.1] that $f_{*}\left(\xi^{i}\right)=1$ if $i=n$ and zero otherwise. The case of singular schemes is the hard part of the theorem because we cannot directly apply the projection formula of [Bloch 1986]. We obtain a proof by an indirect approach of reduction to the smooth case and by unravelling the action of Chern classes on the higher Chow cycles on singular schemes. 
By [Fulton 1984, Lemma 18.2], we can find a closed embedding $\iota: X \rightarrow X^{\prime}$ and a vector bundle $E^{\prime}$ of rank $n+1$ on $X^{\prime}$ such that $E \cong \iota^{*}\left(E^{\prime}\right)$ and $X^{\prime}$ is smooth. We set $Y^{\prime}=\mathbb{P}\left(E^{\prime}\right), \xi^{\prime}=c_{1}\left(\mathscr{O}_{Y^{\prime}}(1)\right)$ and consider the Cartesian diagram

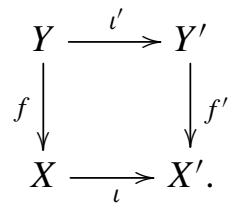

Recall from the construction of the action of $\xi^{\prime i}$ on $\mathrm{CH}_{*}(Y, j)$ in [Bloch 1986] that for any irreducible cycle $[V]$ on $Y \times \Delta^{j}$, the support of $\xi^{i} \cdot[V]=\xi^{i} \cdot[V]$ is $\operatorname{supp}(V \cap \alpha)$, where $\alpha$ is cycle on $Y^{\prime}$ representing $\xi^{\prime i}$ and such that each component of $\alpha$ intersects $V$ properly. This is achieved by using the moving lemma on $Y^{\prime}$, a smooth scheme. Since $\xi^{\prime}$ reduces the dimension of a cycle on $Y \times \Delta^{j}$ by exactly one, we see that $\operatorname{dim}\left(\xi^{i} \cdot f^{*}([W])\right)=\operatorname{dim}(W)+n-i$ and the support of $f_{*}\left(\xi^{i} \cdot f^{*}([W])\right)$ is contained in $W$, whenever $W$ is an irreducible admissible cycle on $X \times \Delta^{j}$. We conclude from the definition of the push-forward map that $f_{*}\left(\xi^{i} \cdot f^{*}([W])\right)$ must be zero if $n-i>0$. Since any admissible cycle on $X \times \Delta^{j}$ is a sum of irreducible admissible cycles, this proves the first case.

We prove the case of $i=n$ by induction on $n$. If $E$ is of rank one, then $f$ is an isomorphism and $O_{Y}(1)$ is trivial and hence $c_{0}\left(O_{Y}(1)\right)=1$. So we assume that $n \geq 1$. We let $\iota: X \hookrightarrow X^{\prime}$ be a closed embedding into a smooth scheme as in (4-2).

By [Panin 2003, Lemma 3.24], there is a morphism $\phi^{\prime}: T^{\prime} \rightarrow X^{\prime}$, which is a composite of projective and affine bundles on $X^{\prime}$ such that $\phi^{\prime *}\left(E^{\prime}\right)=F^{\prime} \oplus L^{\prime}$, where $L^{\prime}$ is a line bundle on $T^{\prime}$. Moreover, if $\phi: T \rightarrow X$ is the restriction of $\phi^{\prime}$ on $X$, then the pull-back map $\phi^{*}: \mathrm{CH}_{*}(X, j) \rightarrow \mathrm{CH}_{*}(T, j)$ is a split injection and the same holds for $\phi^{\prime *}$. Notice here that $X$ is a closed subscheme of $X^{\prime}$ and hence $\mathrm{CH}_{*}(X) \stackrel{\sim}{\longrightarrow} \mathrm{CH}_{*}^{X}\left(X^{\prime}\right)$ in the notation of [ibid., Definition 2.1]. We denote the restrictions of $F^{\prime}$ and $L^{\prime}$ on $X$ by $F$ and $L$ respectively.

Consider the Cartesian diagram

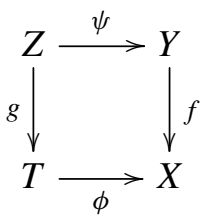

and suppose the given assertion holds for the projective bundle $g$. We then have

$$
\begin{aligned}
\phi^{*}\left(f_{*}\left(\xi^{n} \cdot f^{*}(x)\right)\right) & =g_{*}\left(\psi^{*}\left(\xi^{n} \cdot f^{*}(x)\right)\right)=g_{*}\left(\left(\psi^{*}(\xi)\right)^{n} \cdot \psi^{*} \circ f^{*}(x)\right) \\
& =g_{*}\left(\left(\psi^{*}(\xi)\right)^{n} \cdot g^{*} \circ \phi^{*}(x)\right)=\phi^{*}(x),
\end{aligned}
$$


where the first equality holds by Proposition 2.2 and the last equality holds by our assumption. Since $\phi^{*}$ is injective, we see that the conclusion holds for $f$ as well. Thus we have reduced the problem to the case when $E^{\prime}=F^{\prime} \oplus L^{\prime}$ and $E=F \oplus L$.

If we set $\widetilde{E}=E \otimes L^{-1}$ and $\widetilde{Y}=\mathbb{P}(\widetilde{E})$, there is a commutative diagram

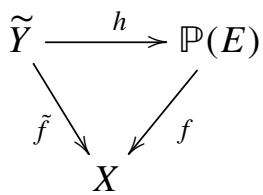

such that $h$ is an isomorphism and $\mathscr{O}_{\widetilde{Y}}(1)=h^{*}\left(\mathcal{O}_{Y}(1)\right) \otimes \tilde{f}^{*}\left(L^{-1}\right)$. Set $\eta=c_{1}(L)$ in $\operatorname{OPCH}^{1}(X, 0)$ and $\tilde{\eta}=\tilde{f}^{*}(\eta)$ and $\tilde{\xi}=c_{1}\left(\mathscr{O}_{\widetilde{Y}}(1)\right)$ in $\operatorname{OPCH}^{1}(\widetilde{Y}, 0)$.

Suppose that our assertion holds for the projective bundle $\tilde{f}$. In this case, we get

$$
f_{*}\left(\xi^{n} \cdot f^{*}(x)\right)=\tilde{f}_{*}\left((\tilde{\eta}+\tilde{\xi})^{n} \cdot \tilde{f}^{*}(x)\right)=\tilde{f}_{*}\left((\tilde{\xi})^{n} \cdot \tilde{f}^{*}(x)\right)=x,
$$

where the last equality holds by our assertion about $\tilde{f}$. The second equality holds because of the fact that

$$
\begin{aligned}
\tilde{f}_{*}\left((\eta+\tilde{\xi})^{n} \cdot \tilde{f}^{*}(x)\right) & =\sum_{i=0}^{n}\left(\begin{array}{c}
n \\
i
\end{array}\right) \tilde{f}_{*}\left((\tilde{\eta})^{i}(\tilde{\xi})^{n-i} \cdot \tilde{f}^{*}(x)\right) \\
& =\sum_{i=0}^{n}\left(\begin{array}{c}
n \\
i
\end{array}\right) \eta^{i} \cdot \tilde{f}_{*}\left((\tilde{\xi})^{n-i} \cdot \tilde{f}^{*}(x)\right)
\end{aligned}
$$

(see [Bloch 1986, Exercise 5.8]) and that the term $\tilde{f}_{*}\left((\tilde{\xi})^{n-i} \cdot \tilde{f}^{*}(x)\right)$ vanishes for $0 \leq n-i<n$ by the first assertion of proposition. Hence, we are further reduced to the case when $E^{\prime}=F^{\prime} \oplus \mathcal{O}_{X^{\prime}}$ and $E=F \oplus \mathcal{O}_{X}$.

Let $Z=\mathbb{P}(F)$ and let $p$ and $q$ be the closed and the open inclusions of $Z \hookrightarrow Y$ and $F \hookrightarrow Y$, respectively. Let $g: Z \hookrightarrow Y \rightarrow X$ be the composite map and set $\zeta=c_{1}\left(O_{Z}(1)\right)$. We observe that $Z$ is a Cartier divisor on $Y$ such that $O_{Y}(1)=\mathscr{L}(Z)$. In particular, the pull-back $p^{*}$ is defined and we have $\xi \cdot f^{*}(a)=p_{*}\left(p^{*} \circ f^{*}(a)\right)$. Since $F$ is of rank $n$, the desired assertion holds for $g: Z \rightarrow X$ by induction. That is, $g_{*}\left(\zeta^{n-1} \cdot g^{*}(x)\right)=x$. We now have

$$
\begin{aligned}
f_{*}\left(\xi^{n} \cdot f^{*}(x)\right) & =f_{*}\left(\xi^{n-1} p_{*}\left(p^{*} \circ f^{*}(x)\right)\right) \\
& =f_{*}\left(p_{*}\left(\zeta^{n-1} \cdot p^{*} \circ f^{*}(x)\right)\right)=g_{*}\left(\zeta^{n-1} \cdot g^{*}(x)\right)=x .
\end{aligned}
$$

This proves the desired assertion and the proof of the proposition is complete.

\section{Demazure operators on equivariant higher Chow groups}

In this section, we introduce Demazure (divided difference) operators on the equivariant higher Chow groups of schemes. Such operators were constructed by Brion 
[1997] on the equivariant Chow groups $\mathrm{CH}_{*}^{G}(X, 0)$ and by Holm and Sjamaar [2008] on the equivariant singular cohomology $H_{G}^{*}(X)$. We extend these operators to the higher Chow groups and discuss some consequences.

Let $G$ be a connected reductive group with a split maximal torus $T$ of rank $n$. Let $\Phi=\Phi(G, T) \subset \mathscr{X}(T)$ be the root system of $G$ with respect to $T$, where $\mathscr{X}(T)$ is the character group of $T$. For any $\alpha \in \Phi$, let $P_{\alpha}$ be the minimal parabolic subgroup of $G$ corresponding to $\alpha$ and let $B=T U_{\alpha} U$ and $B^{\prime}=T U_{-\alpha} U$ be the opposite Borel subgroups of $G$ containing $T$ in $P_{\alpha}$. Let $W_{\alpha}=\left\{s_{\alpha}, s_{-\alpha}\right\}$ denote the Weyl group of $P_{\alpha}$, where $s_{\alpha}$ is the reflection in $\mathscr{L}(T)$ given by

$$
s_{\alpha}(\lambda)=\lambda-\left\langle\alpha^{\vee}, \lambda\right\rangle \alpha \quad \text { for } \lambda \in \mathscr{L}(T) .
$$

Let $X$ be a $k$-scheme with a free $G$-action and let $B$ and $B^{\prime}$ act on $X \times P_{\alpha}$ by $b \cdot(x, g)=(x, b g)$ and $b^{\prime} \cdot(x, g)=\left(b^{\prime} x, g b^{-1}\right)$. It is easy to check that the two actions are free and they commute with each other. Hence we get a free action of $B \times B^{\prime}$ on $X \times G$ by $\left(b, b^{\prime}\right) \cdot(x, g)=\left(b^{\prime} x, b g b^{\prime-1}\right)$. One checks that $B$ acts freely on $X^{\prime}=X \times{ }^{B^{\prime}} P_{\alpha}$. $P_{\alpha}$ acts freely on $X^{\prime}$ by acting trivially on its $X$-factor and by left multiplication on the $P_{\alpha}$-factor. In particular, we have a $B$-equivariant map $X^{\prime} \rightarrow X$ given by $[x, g] \mapsto x$, which yields the projection map on quotients $f_{1}: X^{\prime} / B \rightarrow X / B \cong X^{\prime} / P_{\alpha}$. One also has the $P_{\alpha}$-equivariant map $X^{\prime} \rightarrow X$ given by $[x, g] \mapsto[g x]$, which yields the map $f_{2}: X^{\prime} / B \rightarrow X / B$ on quotients by the action of $B$. It is also easy to check that the data above yield the commutative diagram

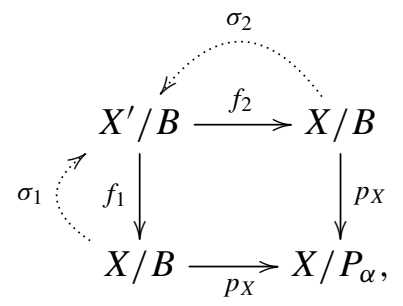

which is Cartesian. The section $\sigma_{2}$ of $f_{2}$ is defined by $\sigma_{2}([x])=[x, 1]$ and the section $\sigma_{1}$ of $f_{1}$ is defined by $\sigma_{1}([x])=\left[x, n_{\alpha}\right]$, where $n_{\alpha}$ is a representative of $s_{\alpha}$ in $N_{P_{\alpha}}(T)$. Since $f_{2}$ is induced by a $P_{\alpha}$-equivariant map, we see that it is $W_{\alpha}$-equivariant with respect to the natural action of $W_{\alpha}$ on $X^{\prime} / B$ and $X / B$. In particular, we get

$$
\begin{aligned}
& f_{2}^{*} \circ s_{\alpha}=s_{\alpha} \circ f_{2}^{*}, \\
& f_{2}^{*}(a x)=a f_{2}^{*}(x) \quad \text { for all } a \in S \text { and } x \in \mathrm{CH}_{*}(X / B) .
\end{aligned}
$$

Note that the all the maps (except the sections) in (5-1) are $P_{\alpha} / B \cong \mathbb{P}^{1}$-bundles and hence they are all smooth and projective. Let $O_{X / B}(1)$ denote the universal quotient line bundle on $X / B$ relative to $p_{X}$. 
Let $D$ denote the image of $\sigma_{1}$ and let $\mathscr{L}(D)$ denote the associated line bundle on $X^{\prime} / B$. Let $\mathscr{L}_{\lambda}$ and $\mathscr{L}_{\lambda}^{\prime}$ denote the line bundles $X \times{ }^{B} L_{\lambda}$ and $X^{\prime} \times{ }^{B} L_{\lambda}$ on $X / B$ and $X^{\prime} / B$ respectively, where $L_{\lambda}$ is the $B$-equivariant line bundle on $\operatorname{Spec}(k)$ corresponding to the character $\lambda$ of $T$. It follows from [Demazure 1974, Lemme 2, Proposition 2] that

$$
\begin{aligned}
\mathscr{L}_{\alpha}^{\prime} & =f_{1}^{*}\left(\mathscr{L}_{\alpha}\right) \otimes(\mathscr{L}(D))^{\otimes 2}, \\
s_{\alpha}(\mathscr{L}(D)) & =\left(\mathscr{L}(D) \otimes f_{1}^{*}\left(\mathscr{L}_{\alpha}\right)\right)^{\otimes(-1)} .
\end{aligned}
$$

5a. Demazure operators on $\mathbf{C H}_{*}(\boldsymbol{X})$. Let $G$ be a connected reductive group with split maximal torus $T$ as above and let $X$ be a $G$-scheme of dimension $d$. Let $j \leq d$ be an integer and let $(V, U)$ be a good pair for the $G$-action corresponding to $d-j$. The smooth and projective morphism $p_{X \times U}: X_{B} \rightarrow X_{P_{\alpha}}$ yields the maps

$$
p_{X \times U}^{*}: \mathrm{CH}_{j}^{P_{\alpha}}(X, i) \rightarrow \mathrm{CH}_{j}^{B}(X, i) \text { and } p_{X \times U *}: \mathrm{CH}_{j}^{B}(X, i) \rightarrow \mathrm{CH}_{j+1}^{P_{\alpha}}(X, i) \text {. }
$$

For the rest of this text, the ring $S$ will denote the equivariant Chow ring

$$
S(T)=\mathrm{CH}_{T}^{*}(k, 0) \text {. }
$$

Lemma 5.1. The maps $p_{X \times U}^{*}$ and $p_{X \times U *}$ do not depend on the choice of the good $\operatorname{pair}(V, U)$.

Proof. We prove the lemma for the push-forward map and a very similar proof works also for the pull-back map; see [Edidin and Graham 1998, Section 1].

Let $g$ and $b$ denote the dimensions of $P_{\alpha}$ and $B$, respectively. Let $(V, U)$ and $\left(V^{\prime}, U^{\prime}\right)$ be good pairs of dimensions $l$ and $l^{\prime}$, respectively, corresponding to $d-j$. We let $\mathscr{V}=V \oplus V^{\prime}$ and $\mathscr{U}=\left(U \oplus V^{\prime}\right) \cup\left(V \oplus U^{\prime}\right)$. Let $G$ act diagonally on $\mathscr{V}$. Then it is easy to see that the dimension of the complement of the open subset $X \times{ }^{B}\left(U \oplus V^{\prime}\right)$ in $X \times{ }^{B} \cup$ is sufficiently smaller than $l+l^{\prime}-b+j$. Similarly, the dimension of the complement of the open subset $X \times{ }^{P_{\alpha}}\left(U \oplus V^{\prime}\right)$ in $X \times{ }^{p_{\alpha}} \mathcal{U}$ is sufficiently smaller than $l+l^{\prime}-g+j \leq l+l^{\prime}-b+j$. It follows from the localization sequence for the higher Chow groups and Lemma 5.2 that the there is a commutative diagram

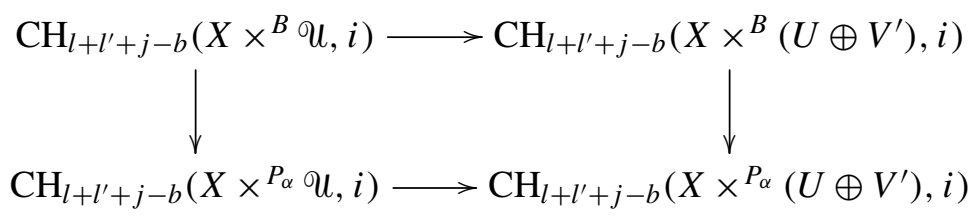

where the vertical maps are the push-forward maps and the horizontal maps are isomorphisms.

On the other hand, the maps

$$
X \times{ }^{G}\left(U \oplus V^{\prime}\right) \rightarrow X \times{ }^{G} U \text { and } X \times{ }^{B}\left(U \oplus V^{\prime}\right) \rightarrow X \times{ }^{B} U
$$


are vector bundles of rank $l^{\prime}$ and hence we get another commutative diagram

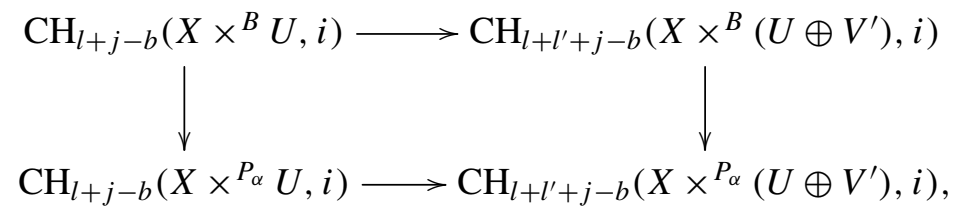

where the vertical arrows are the push-forward maps and the horizontal arrows are isomorphisms by the homotopy invariance.

Combining the two isomorphisms above, we get the commutative diagram

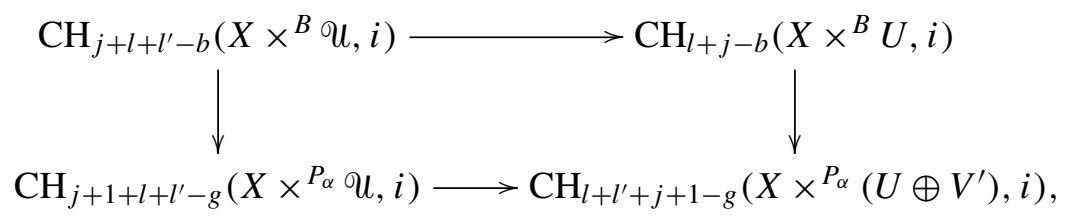

where the horizontal maps are isomorphisms. By repeating the same argument with $U^{\prime}$, we get the diagram above with $U$ replaced by $U^{\prime}$ and $V^{\prime}$ replaced by $V$ on the right column. This proves the lemma.

Lemma 5.2. Let $p: X \rightarrow Y$ be a morphism in $\mathscr{V}_{P_{\alpha}}$ such that $P_{\alpha}$ acts freely on $X$ and $Y$. Then the diagram of quotients

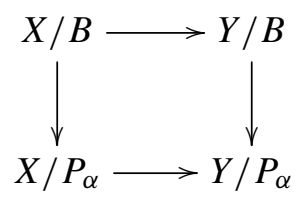

is Cartesian such that the vertical maps are smooth and projective.

Proof. This is an easy exercise.

Proposition 5.3. For any $X \in \mathscr{V}_{G}$, one has the restriction and the push-forward maps

$$
r_{B, X}^{P_{\alpha}}: \mathrm{CH}_{*}^{P_{\alpha}}(X, i) \rightarrow \mathrm{CH}_{*}^{B}(X, i) \quad \text { and } \quad p_{B, X}^{P_{\alpha}}: \mathrm{CH}_{*}^{B}(X, i) \rightarrow \mathrm{CH}_{*+1}^{P_{\alpha}}(X, i) .
$$

These maps are contravariant with respect to the flat maps and covariant with respect to the proper morphisms of schemes in $\mathscr{V}_{G}$.

Proof. Let $j \leq d$ be an integer and let $(V, U)$ be a good pair for the $G$-action corresponding to $d-j$. We define $r_{B, X}^{P_{\alpha}}$ and $p_{B, X}^{P_{\alpha}}$ to be $p_{X \times U}^{*}$ and $p_{X \times U *}$ respectively. It follows from Lemma 5.1 that these maps are well-defined.

The functoriality properties of $r_{B, X}^{P_{\alpha}}$ is already known; see [Edidin and Graham 1998, Section 1]. To prove these properties for $p_{B, X}^{P_{\alpha}}$, it suffices to prove the same 
for $p_{X \times U_{*}}$. But this follows easily from Lemma 5.2 and the similar properties of the ordinary higher Chow groups.

The following result generalizes [Brion 1997, Theorem 6.3] to equivariant higher Chow groups.

Theorem 5.4. Let $\alpha$ be a simple root of $G$. For any $X \in \mathscr{V}_{G}$ and $i \geq 0$, there is a unique operator $\delta_{\alpha}^{X}$ on $\mathrm{CH}_{*}^{T}(X, i)$ such that for all $u \in S$ and $v \in \mathrm{CH}_{*}^{T}(X, i)$, we have

(1) $\alpha \delta_{\alpha}^{X}(v)=v-s_{\alpha}(v)$ and

(2) $\delta_{\alpha}^{X}(u v)=u \delta_{\alpha}^{X}(v)+\delta_{\alpha}^{k}(u) s_{\alpha}(v)$ if $X$ is smooth.

Moreover, $\delta_{\alpha}^{X}$ commutes with the G-equivariant flat pull-back and proper pushforward maps between $T$-equivariant higher Chow groups.

Proof. Let $B$ and $B^{\prime}$ be the opposite Borel subgroups of $P_{\alpha}$ containing $T$. Using Proposition 3.3, we can replace $T$ by $B$ to define $\delta_{\alpha}^{X}$. We let

$$
\delta_{\alpha}^{X}:=r_{B, X}^{P_{\alpha}} \circ p_{B, X}^{P_{\alpha}}: \mathrm{CH}_{j}^{B}(X, i) \rightarrow \mathrm{CH}_{j+1}^{B}(X, i) .
$$

The co- and contravariant functoriality of $\delta_{\alpha}^{X}$ follows from Proposition 5.3. The uniqueness of $\delta_{\alpha}^{X}$ follows from [Brion 1997, Theorem 6.3] since this definition of $\delta_{\alpha}^{X}$ coincides with the one defined for $\mathrm{CH}_{*}^{B}(X, 0)$ by Brion. We only need to show the first and the second assertions.

Let $j \leq \operatorname{dim}(X)$ and $(V, U)$ be a good pair the $G$-action corresponding to $d-j$. There is a $G$-equivariant projection $X \times U \rightarrow X$ such that

$$
\mathrm{CH}_{j}^{G}(X, i) \stackrel{\sim}{\longrightarrow} \mathrm{CH}_{j}^{G}(X \times U, i)
$$

and $\delta_{\alpha}^{X}$ on $\mathrm{CH}_{j}^{G}(X, i)$ coincides with the operator $\delta_{\alpha}^{X \times U}$ on $\mathrm{CH}_{j}^{G}(X \times U, i)$ by its construction. Hence we can assume that $G$ acts freely on $X$.

We now consider the diagram (5-1). Since $f_{2}$ is a $\mathbb{P}^{1}$-bundle, the map $f_{2}^{*}$ is split injective by Proposition 4.6. Hence it suffices to show that the two assertions of the theorem hold after applying $f_{2}^{*}$.

On the other hand, $f_{2}^{*}\left(s_{\alpha}(v)\right)=s_{\alpha}\left(f_{2}^{*}(v)\right)$ and $f_{2}^{*}\left(\alpha \delta_{\alpha}^{X}(v)\right)=\alpha f_{2}^{*}\left(\delta_{\alpha}^{X}(v)\right)$ by (5-2). Since $f_{2}$ is induced by a $G$-equivariant map $X^{\prime} \rightarrow X$, we also have $f_{2}^{*}\left(\delta_{\alpha}^{X}(v)\right)=\delta_{\alpha}^{X^{\prime}}\left(f_{2}^{*}(v)\right)$ by the functoriality of $\delta_{\alpha}$. Thus we need to show for $u \in S$ and $v^{\prime} \in \mathrm{CH}_{*}\left(X^{\prime} / B, i\right)$ that

$$
\alpha \delta_{\alpha}^{X^{\prime}}\left(v^{\prime}\right)=v^{\prime}-s_{\alpha}\left(v^{\prime}\right) \text { and } \delta_{\alpha}^{X^{\prime}}\left(u v^{\prime}\right)=u \delta_{\alpha}^{X^{\prime}}\left(v^{\prime}\right)+\delta_{\alpha}^{k}(u) s_{\alpha}\left(v^{\prime}\right) \text { if } X \in \mathscr{V}_{G}
$$

Let $c: \mathrm{CH}_{T}^{*}(k, 0) \cong \mathrm{OPCH}_{T}^{*}(k, 0) \rightarrow \mathrm{OPCH}^{*}(X / B, 0)$ be the ring homomorphism on the operational Chow groups induced by the map on the Picard groups $L_{\alpha} \mapsto \mathscr{L}_{\alpha}$. We denote the corresponding map $\mathrm{OPCH}_{T}^{*}(k, 0) \rightarrow \mathrm{OPCH}^{*}\left(X^{\prime} / B, 0\right)$ by $c^{\prime}$. We set $\xi=c_{1}(\mathscr{L}(D))$ and $\zeta=c_{1}\left(\mathcal{O}_{X^{\prime} / B}(1)\right)$, where $\mathscr{O}_{X^{\prime} / B}(1)$ is the universal 
quotient line bundle associated to the $\mathbb{P}^{1}$-bundle $f_{1}$. We shall write $\delta_{\alpha}^{k}$ simply as $\delta_{\alpha}$ in what follows.

Since $\mathscr{L}(D)$ and $\mathscr{O}_{X^{\prime} / B}(1)$ have same degree on every fiber of $f_{1}$, there is a line bundle $\mathcal{M}$ on $X / B$ such that $O_{X^{\prime} / B}(1) \cong \mathscr{L}(D) \otimes f_{1}^{*}(\mathcal{M})$. In particular, there exists $\eta \in \operatorname{OPCH}^{1}(X / B, 0)$ such that $\zeta=\xi+f_{1}^{*}(\eta)$. Since $f_{1}^{*}$ commutes with the action of $\mathrm{OPCH}^{*}(X / B, 0)$ on the higher Chow groups, it follows from Proposition 4.6 that we can write $v^{\prime} \in \mathrm{CH}_{*}\left(X^{\prime} / B, i\right)$ as

$$
v^{\prime}=f_{1}^{*}(a)+\xi f_{1}^{*}(b), \quad \text { with } a, b \in \mathrm{CH}_{*}(X / B, i) .
$$

Furthermore, it also follows that for any $b \in \mathrm{CH}_{*}(X / B, i)$,

$$
f_{1 *}\left(\xi f_{1}^{*}(b)\right)=f_{1 *}\left(\zeta f_{1}^{*}(b)\right)-f_{1 *}\left(f_{1}^{*}(\eta b)\right)=b+0=b .
$$

Since $s_{\alpha}$ keeps the elements of the form $f^{*}(a)$ invariant, we get

$$
\begin{aligned}
s_{\alpha}\left(v^{\prime}\right) & =f_{1}^{*}(a)+s_{\alpha}(\xi) f_{1}^{*}(b) \\
& =f_{1}^{*}(a)-\left[\xi+f_{1}^{*}(c(\alpha))\right] f_{1}^{*}(b),
\end{aligned}
$$

where the second equality follows from (5-3).

On the other hand, we have seen in (5-1) that $f_{1}$ is same as the quotient map $X^{\prime} / B \rightarrow X^{\prime} / P_{\alpha}$ and hence $\delta_{\alpha}^{X^{\prime}}=f_{1}^{*} f_{1_{*}}$. This yields

$$
\begin{aligned}
v^{\prime}-c^{\prime}(\alpha) \delta_{\alpha}^{X^{\prime}}\left(v^{\prime}\right) & =f_{1}^{*}(a)+\xi f_{1}^{*}(b)-c^{\prime}(\alpha) f_{1}^{*}\left[f_{1 *} f_{1}^{*}(a)+f_{1 *}\left(\xi f_{1}^{*}(b)\right)\right] \\
& ={ }^{\dagger} f_{1}^{*}(a)+\xi f_{1}^{*}(b)-c^{\prime}(\alpha) f_{1}^{*}(b) \\
& ={ }^{\ddagger} f_{1}^{*}(a)+\xi f_{1}^{*}(b)-\left[f_{1}^{*}(c(\alpha))+2 \xi\right] f_{1}^{*}(b) \\
& =f_{1}^{*}(a)-\left[\xi+f_{1}^{*}(c(\alpha))\right] f_{1}^{*}(b),
\end{aligned}
$$

where $={ }^{\dagger}$ follows from Proposition 4.6 and (5-8), and $={ }^{\dagger}$ follows from (5-3). The first equality of (5-6) follows at once by comparing (5-9) and (5-10).

To prove the second equality of (5-6) for $u \in S$ and $v^{\prime} \in \mathrm{CH}_{*}\left(X^{\prime} / B, i\right)$ with $X$ smooth, we can assume using (5-7) that $v^{\prime}$ is either $f_{1}^{*}(a)$ or $\xi f_{1}^{*}(a)$. We now have

$$
\begin{aligned}
\delta_{\alpha}^{X}\left(u f_{1}^{*}(a)\right) & =f_{1}^{*} \circ f_{1_{*}}\left(u f_{1}^{*}(a)\right)={ }^{1} f_{1}^{*}\left(f_{1 *}(u) \cdot a\right)=f_{1}^{*} f_{1_{*}}(u) \cdot f_{1}^{*}(a) \\
& ={ }^{2} \delta_{\alpha}^{k}(u) \cdot f_{1}^{*}(a)=\delta_{\alpha}^{k}(u) \cdot s_{\alpha}\left(f_{1}^{*}(a)\right)+u \delta_{\alpha}^{X}\left(f_{1}^{*}(a)\right) .
\end{aligned}
$$

The equality $={ }^{1}$ holds by the projection formula for smooth schemes (see [Bloch 1986, Exercise 5.8]) and $=^{2}$ holds by [Brion 1997, Theorem 6.3]. The last equality holds because $f_{1}^{*}(a)$ is invariant under $s_{\alpha}$ and $f_{1}^{*} f_{1_{*}}(a)$ vanishes, again by the projection formula. The required formula for $v^{\prime}=\xi f_{1}^{*}(a)$ is proved exactly in the similar way using the observation that $f_{1_{*}}(\xi)=1$ and that the equality $={ }^{2}$ holds even if we replace $u$ by $\xi u$. 
Proposition 5.5. For $X \in \mathscr{V}_{G}$, let $r_{T, X}^{G}: \mathrm{CH}_{*}^{G}(X, i) \rightarrow \mathrm{CH}_{*}^{T}(X, i)$ be the restriction map.

(1) $\delta_{\alpha} \circ r_{T, X}^{G}=0$.

(2) If $X$ is smooth, then $\delta_{\alpha}$ is $\mathrm{CH}_{G}^{*}(X)$-linear.

(3) $\delta_{\alpha}^{2}=0$.

(4) $s_{\alpha} \delta_{\alpha}=\delta_{\alpha}=-\delta_{-\alpha}, \delta_{\alpha} s_{\alpha}=-\delta_{\alpha}$.

Proof. Since $r_{T, X}^{G}=r_{T, X}^{P_{\alpha}} \circ r_{P_{\alpha}, X}^{G}$, we can replace $G$ by $P_{\alpha}$. It suffices then to show that

$$
p_{B, X}^{P_{\alpha}} \circ r_{B, X}^{P_{\alpha}}=0 \text {. }
$$

But this follows immediately from Proposition 4.6. The second point follows from the observation that $f_{1}^{*}$ and $f_{1 *}$ in $(5-1)$ are $\mathrm{CH}^{*}\left(X^{\prime} / G\right)$-linear. The third point follows directly from the first and the fourth point is an immediate consequence of the other assertions of the proposition.

5b. Ring of Demazure operators. Let $\left\{\alpha_{1}, \ldots, \alpha_{m}\right\}$ be the set of all simple roots of $G$. For any sequence $I=\left\{i_{1}, \ldots, i_{l}\right\}$ of integers in the interval $[1, m]$, we define the operator $\delta_{I}^{X}$ on $\mathrm{CH}_{*}^{T}(X, i)$ by

$$
\delta_{I}^{X}=\delta_{\alpha_{i_{l}}} \circ \cdots \circ \delta_{\alpha_{i_{1}}} .
$$

Following the notation of [Brion 1997, §6.4], we let D denote the subring of $\operatorname{End}_{\mathbb{Z}}(S)$ generated by the elements $\delta_{\alpha}^{k}$ and the endomorphisms given by the multiplication in $S$. It is clear from the definition of $\mathbf{D}$ and Theorem 5.4 that $\mathbf{D}$ contains the twisted group algebra $S[W]$ and there are inclusions of rings $S \subsetneq S[W] \subsetneq \mathbf{D}$. It is known that $\mathbf{D}$ is a free $S$-module with basis $\left\{\partial_{w}\right\}_{w \in W}$, where $\partial_{w}$ is same as $\delta_{I}^{k}$ above whenever $w=s_{\alpha_{i_{1}}} \cdots s_{\alpha_{i_{l}}}$. As an immediate consequence of Theorem 5.4, Proposition 5.5 and (5-11), we get:

Corollary 5.6. For any $X \in \mathscr{V}_{G}$ and $i \geq 0$, there is a unique $\mathbf{D}$-module structure on $\mathrm{CH}_{*}^{T}(X, i)$, which extends the action of $S[W]$. Moreover, the flat pull-back and proper push-forward maps between the T-equivariant higher Chow groups are $\mathbf{D}$-linear. For $X \in \mathscr{V}_{G}^{S}$, the $\mathbf{D}$-module structure on $\mathrm{CH}_{T}^{*}(X)$ commutes with its $\mathrm{CH}_{G}^{*}(X)$-module structure.

Let $I(\mathbf{D})$ be the subset of $\mathbf{D}$ consisting of those operators $\delta$ such that $\delta(1)=0$. It is easy to check that $I(\mathbf{D})$ is a left ideal of $\mathbf{D}$ generated by $\left\{\partial_{w}\right\}_{w \neq 1}$. For any $X \in \mathscr{V}_{G}$, let

$$
\left(\mathrm{CH}_{*}^{T}(X, i)\right)^{I(\mathbf{D})}=\left\{x \in \mathrm{CH}_{*}^{T}(X, i) \mid \delta(x)=0 \forall \delta \in I(\mathbf{D})\right\} .
$$


Since the Weyl group is generated by simple reflections, it follows from Theorem 5.4 and Proposition 5.5 that

$$
r_{T, X}^{G}\left(\mathrm{CH}_{*}^{G}(X, i)\right) \subseteq\left(\mathrm{CH}_{*}^{T}(X, i)\right)^{I(D)} \subseteq\left(\mathrm{CH}_{*}^{T}(X, i)\right)^{W} .
$$

Recall that the torsion index $t_{G}$ of the group $G$ is the order of the cokernel of the map $S_{N} \rightarrow \mathrm{CH}^{N}(G / B)$, where $N=\operatorname{dim}(G / B)=\left|\Phi^{+}\right|$. Let $R$ denote the localized $\operatorname{ring} \mathbb{Z}\left[t_{G}^{-1}\right]$.

Theorem 5.7. Let $X \in \mathscr{V}_{G}^{S}$ and $i \geq 0$ be such that $\mathrm{CH}_{T}^{*}(X, i)$ is torsion-free. Then the map $\mathrm{CH}_{*}^{G}(X, i) \rightarrow\left(\mathrm{CH}_{*}^{T}(X, i)\right)^{W}$ is an isomorphism over $R$.

Proof. Let $B$ be a Borel subgroup of $G$ containing $T$. Let $(V, U)$ be a good pair for the $G$-action and consider the Cartesian diagram

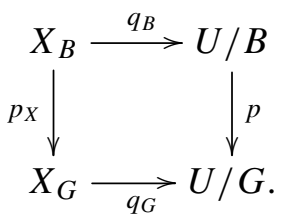

By the definition of $t_{G}$, it follows that there is $a \in \mathrm{CH}^{N}(U / B, 0)$ such that $p_{*}(a)=t_{G} \in \mathrm{CH}^{0}(U / G, 0)$. Using the projection formula, we get for any $x \in$ $\mathrm{CH}^{*}\left(X_{G}, i\right)$,

$$
p_{X_{*}}\left(q_{B}^{*}(a) p_{X}^{*}(x)\right)=p_{X_{*}}\left(q_{B}^{*}(a)\right) x=q_{G}^{*}\left(p_{*}(a)\right) x=t_{G} x .
$$

In particular, $r_{T, X}^{G}$ is split injective over $R$.

To show the surjectivity, we see from the above that for any $x \in \mathrm{CH}^{*}\left(X_{G}, i\right)$,

$$
p_{X}^{*} f p_{X}^{*}(x)=p_{X}^{*}\left(t_{G} x\right)=t_{G} p_{X}^{*}(x),
$$

where $f(y)=p_{X_{*}}\left(\right.$ ay). This in particular implies that $p_{X}^{*} f(y)=t_{G} y$ for all $y$ in the image of $p_{X}^{*}$. It follows from Corollary 8.7 that

$$
p_{X}^{*} f(y)=t_{G} y \text { for all } y \in\left(\mathrm{CH}^{*}\left(X_{B}, i\right)_{\mathbb{Q}}\right)^{W} .
$$

Since $\mathrm{CH}_{T}^{*}(X, i)$ is torsion-free, we must have

$$
p_{X}^{*} f(y)=t_{G} y \quad \text { for all } y \in\left(\mathrm{CH}^{*}\left(X_{B}, i\right)\right)^{W} .
$$

Hence the map $r_{T, X}^{G}$ is surjective onto the $W$-invariants over $R$.

Remark 5.8. The proof of the theorem above in fact shows that the map $r_{T, X}^{G}$ is split injective over $R$ for any $X \in \mathscr{V}_{G}^{S}$. 
Corollary 5.9. Let $X$ be a smooth projective scheme with a $G$-action such that the fixed point locus $X^{T}$ for the T-action is isolated. Then the map

$$
\mathrm{CH}_{*}^{G}(X, 0) \rightarrow\left(\mathrm{CH}_{*}^{T}(X, 0)\right)^{W}
$$

is an isomorphism over $R$.

Proof. This is an immediate consequence of [Krishna 2009a, Theorem 4.2] and Theorem 5.7.

\section{The Leray-Hirsch Theorem}

In algebraic topology, the Leray-Hirsch theorem is a very important tool for describing the cohomology of the total space of a fiber bundle. Since the arguments in this theorem are mostly topological, one cannot always expect such results for the cohomology theories of algebraic varieties. A version of the Leray-Hirsch theorem was proven for the Chow groups of the total space of a Zariski-locally trivial fibration in [Ellingsrud and Strømme 1989, Lemma 2.8; Edidin and Graham 1997, Lemma 6]. In this section, we prove the general form of the Leray-Hirsch theorem for the higher Chow groups of schemes. We shall give several important applications of this theorem in the next few sections.

6a. A Künneth formula. In [Fulton 1984, Example 1.9.1], a $k$-scheme $L$ is called cellular if it has a filtration $\varnothing=L_{n+1} \subsetneq L_{n} \subsetneq \cdots \subsetneq L_{1} \subsetneq L_{0}=L$ by closed subschemes such that each $L_{i} \backslash L_{i+1}$ is an affine space $\mathbb{A}_{k}^{r_{i}} \cdot{ }^{1}$ It follows from the Bruhat decomposition that schemes of the type $G / B$ are cellular, where $B$ is a Borel subgroup of a split reductive group $G$.

Lemma 6.1. Let $L$ be a cellular scheme with the cellular decomposition

$$
\varnothing=L_{n+1} \subsetneq L_{n} \subsetneq \cdots \subsetneq L_{1} \subsetneq L_{0}=L
$$

and let $U_{i}=L \backslash L_{i}$ for $0 \leq i \leq n+1$. Then for any $0 \leq i \leq n$ and $p \geq 0$, the sequence

$$
0 \rightarrow \mathrm{CH}_{*}\left(U_{i+1} \backslash U_{i}, p\right) \rightarrow \mathrm{CH}_{*}\left(U_{i+1}, p\right) \rightarrow \mathrm{CH}_{*}\left(U_{i}, p\right) \rightarrow 0
$$

is exact.

Proof. The proof is very similar to the arguments of [Kahn 1999, Lemma 3.3] using an induction on the number of cells.

Lemma 6.2. Let $L$ be a cellular scheme and let $X$ be a any $k$-scheme. Then the exterior product map

$$
\mathrm{CH}_{*}(X) \otimes_{\mathbb{Z}} \mathrm{CH}_{*}(L, 0) \rightarrow \mathrm{CH}_{*}(X \times L)
$$

${ }^{1}$ Some authors allow $L_{i} \backslash L_{i+1}$ to be a disjoint union of affine spaces over $k$. But both definitions are equivalent. 
is an isomorphism. In particular, the natural map $\mathrm{CH}^{*}(k) \otimes_{\mathbb{Z}} \mathrm{CH}_{*}(L, 0) \rightarrow \mathrm{CH}_{*}(L)$ is an isomorphism.

Proof. Consider the cellular decomposition of $L$ as in Lemma 6.1. Then each $U_{i}=L \backslash L_{i}$ is also a cellular scheme. It suffices to show by induction that (6-1) holds when $L$ is any of these $U_{i}$. There is nothing to prove for $i=0$ and the case $i=1$ follows by the homotopy invariance since $U_{1}$ is an affine space. In general, we have the short exact sequence

$$
0 \rightarrow \mathrm{CH}_{*}\left(U_{i+1} \backslash U_{i}, 0\right) \rightarrow \mathrm{CH}_{*}\left(U_{i+1}, 0\right) \rightarrow \mathrm{CH}_{*}\left(U_{i}, 0\right) \rightarrow 0
$$

by applying Lemma 6.1 with $p=0$. Since each $U_{i+1} \backslash U_{i}$ is an affine space, it also follows from Lemma 6.1 and by induction on the number of affine cells that each $\mathrm{CH}_{*}\left(U_{i}, 0\right)$ is a free abelian group of finite rank. Tensoring this with $\mathrm{CH}_{*}(X)$ over $\mathrm{CH}^{*}(k, 0)=\mathbb{Z}$, we get a commutative diagram

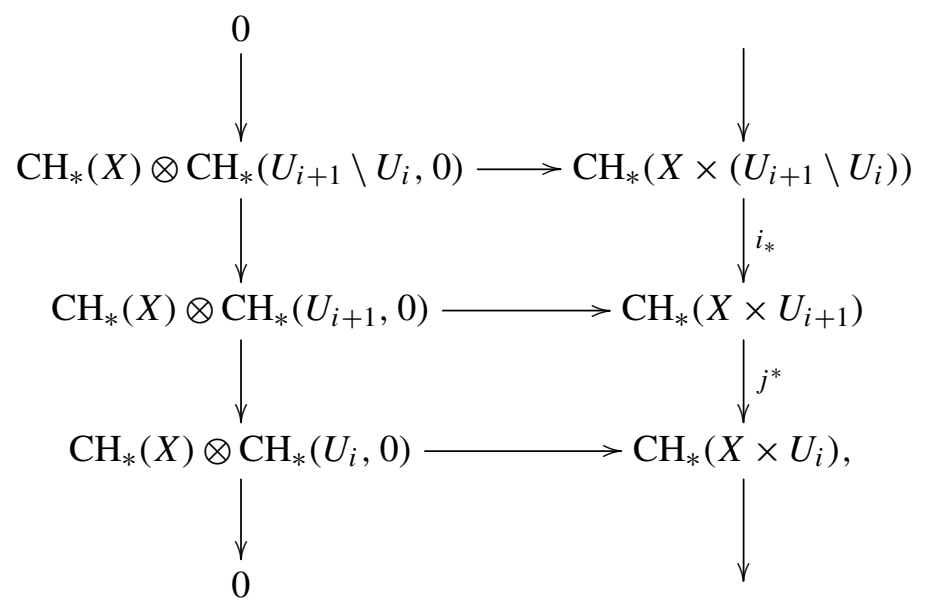

where the left column is exact by the freeness of each $\mathrm{CH}_{*}\left(U_{i}, 0\right)$ and the right column is the localization exact sequence. The top horizontal arcolumn is an isomorphism by the homotopy invariance and the bottom horizontal arcolumn is an isomorphism by the induction. In particular, $j^{*}$ is surjective in all indices. We conclude that $i_{*}$ is injective in all indices and the middle horizontal arcolumn is also an isomorphism.

6b. Leray-Hirsch with integral coefficients. Let $F$ be a cellular scheme over $k$. For any field extension $k \hookrightarrow l$, the scheme $F_{l}$ is also cellular, for which the cellular decomposition and the affine cells are the base extensions of the cellular decomposition and affine cells of $F$. It follows from Lemma 6.1 that the map $\mathrm{CH}_{*}(F, 0) \rightarrow \mathrm{CH}_{*}\left(F_{l}, 0\right)$ is an isomorphism. The following is the integral version of the Leray-Hirsch theorem for the Zariski-locally trivial fibrations. 
Theorem 6.3. Let $B$ be a smooth $k$-scheme and let $F \stackrel{i}{\rightarrow} E \stackrel{p}{\rightarrow} B$ be a Zariskilocally trivial fibration such that the fiber $F$ is a smooth cellular scheme. Assume that there are elements $\left\{e_{1}, \ldots, e_{r}\right\}$ in $\mathrm{CH}^{*}(E, 0)$ such that

$$
\left\{f_{1}=i^{*}\left(e_{1}\right), \ldots, f_{r}=i^{*}\left(e_{r}\right)\right\}
$$

forms a $\mathbb{Z}$-basis of $\mathrm{CH}^{*}\left(F_{y}, 0\right)$ for each fiber $F_{y}$ of the fibration. Then the map

$$
\Phi: \mathrm{CH}^{*}(F, 0) \otimes_{\mathbb{Z}} \mathrm{CH}^{*}(B) \rightarrow \mathrm{CH}^{*}(E), \quad \sum_{1 \leq i \leq r} f_{i} \otimes b_{i} \mapsto \sum_{1 \leq i \leq r} p^{*}\left(b_{i}\right) e_{i}
$$

is an isomorphism. In particular, $\mathrm{CH}^{*}(E)$ is a free $\mathrm{CH}^{*}(B)$-module with basis $\left\{e_{1}, \ldots, e_{r}\right\}$.

Proof. Since $k$ is perfect, we can find a filtration

$$
\varnothing=B_{n+1} \subsetneq B_{n} \subsetneq \cdots \subsetneq B_{1} \subsetneq B_{0}=B
$$

of $B$ by closed subschemes such that for each $0 \leq i \leq n$, the scheme $B_{i} \backslash B_{i+1}$ is smooth and the given fibration is trivial over it. We set $U_{i}=B \backslash B_{i}$ and $V_{i}=U_{i} \backslash U_{i-1}=B_{i-1} \backslash B_{i}$. Observe then that each of the $U_{i}$ and $V_{i}$ is smooth. Set $E_{i}=p^{-1}\left(U_{i}\right)$ and $W_{i}=p^{-1}\left(V_{i}\right)$. We prove by induction on $i$ that the map $\mathrm{CH}^{*}(F, 0) \otimes_{\mathbb{Z}} \mathrm{CH}^{*}\left(U_{i}\right) \rightarrow \mathrm{CH}^{*}\left(E_{i}\right)$ is an isomorphism, which will prove the theorem. Since $U_{0}=\varnothing$ and $E_{1}=U_{1} \times F$, the desired isomorphism for $i=1$ follows from Lemma 6.2. We now consider the commutative diagram

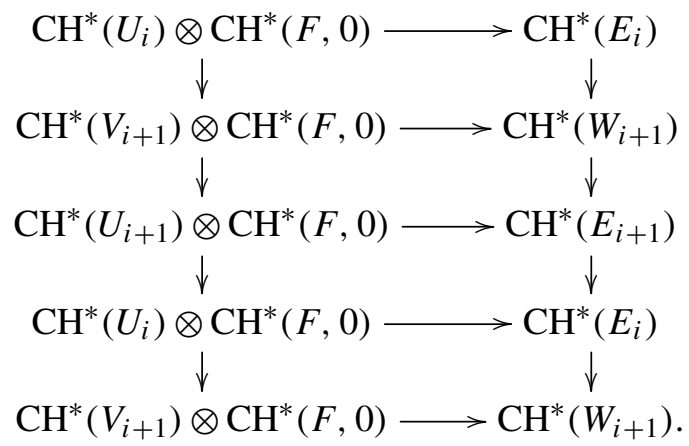

The left column is obtained by tensoring the long exact localization sequence for higher Chow groups with $\mathrm{CH}^{*}(F, 0)$ over $\mathbb{Z}$, and the right column is just the localization exact sequence. Since $\mathrm{CH}^{*}(F, 0)$ is a free abelian group, the left column is also exact.

It is easily checked that the second and the third squares commute using the commutativity property of the push-forward and pull-back maps of higher Chow groups in a fiber diagram. We show that the other squares also commute. It is enough to show that the first square commutes as the fourth one is same as the first. 
Let $\delta$ denote the connecting homomorphism in a long exact localization sequence for the higher Chow groups.

Before we show the required commutativity, let us recall that if $(X, Y)$ is pair of $k$-schemes where $i: Y \hookrightarrow X$ is a closed subscheme with complement $j: U \hookrightarrow X$, then the localization exact sequence is the long exact homology sequence associated to the short exact sequence of cycle complexes

$$
0 \rightarrow \mathscr{E}_{n}(Y, \bullet) \stackrel{i_{*}}{\rightarrow} \mathscr{E}_{n}(X, \bullet) \stackrel{j^{*}}{\rightarrow} \frac{\mathscr{L}_{n}(X, \bullet)}{\mathscr{L}_{n}(Y, \bullet)} \rightarrow 0
$$

where the natural map

$$
\frac{\mathscr{E}_{n}(X, \bullet)}{\mathscr{E}_{n}(Y, \bullet)} \rightarrow \mathscr{E}_{n}(U, \bullet)
$$

is a quasi-isomorphism. So we identify the last term with $\mathscr{L}_{n}(U, \bullet)$. The formalism of the homological algebra now shows that the connecting homomorphism $\delta: \mathrm{CH}_{n}(U, i) \rightarrow \mathrm{CH}_{n}(Y, i-1)$ is obtained as one obtains the connecting homomorphism in the snake lemma. In particular, this is same as the differential map $\partial: \mathscr{E}_{n}(X, i) \rightarrow \mathscr{E}_{n}(X, i-1)$, evaluated on the homology groups. The Leibniz rule for this differential now implies that the connecting homomorphism $\delta$ also satisfies the Leibniz rule; see [Panin 2003, §2.4].

If we now start with an element $b \otimes i^{*}\left(e_{j}\right) \in \mathrm{CH}^{*}\left(U_{i}\right) \otimes \mathrm{CH}^{*}(F, 0)$ and map this vertically, we get $\delta b \otimes i^{*}\left(e_{j}\right)$, which maps horizontally down to $p^{*}(\delta b) \cdot e_{j}$. On the other hand, if we first map horizontally, we get $p^{*}(b) \cdot e_{j}$ which maps vertically to $\delta\left(p^{*}(b) \cdot e_{j}\right)$. Using the Leibniz rule above, this last term is same as $\delta p^{*}(b) \cdot e_{j}=p^{*}(\delta b) \cdot e_{j}$ since $\delta e_{j}=0$. We have shown that the diagram above commutes.

The first and the fourth horizontal arrows in (6-3) are isomorphisms by induction. The second and the fifth horizontal arrows are isomorphisms by Lemma 6.2. Hence the middle horizontal arrow is also an isomorphism by the 5-lemma.

6c. Leray-Hirsch with rational coefficients. We need the following step to prove the rational version of the Leray-Hirsch theorem for the étale locally trivial fibrations of smooth schemes.

Let $F$ be a smooth cellular scheme over $k$. We have seen before that for any field extension $k \hookrightarrow l$, the natural map $\mathrm{CH}^{*}(F, 0) \rightarrow \mathrm{CH}^{*}\left(F_{l}, 0\right)$ is an isomorphism. Moreover, each of these is a free abelian group with the basis vectors given by the closures of the affine cells in the cellular decomposition. We fix this basis $\left\{f_{1}, \ldots, f_{r}\right\}$ of $\mathrm{CH}^{*}\left(F_{l}, 0\right)$ in what follows. For a complete flag variety $G / B$, this set is same as the set of Schubert cycles $\left\{\zeta_{w}\right\}_{w \in W}$. 
Lemma 6.4. Let $B$ be a smooth $k$-scheme and let $F \stackrel{i}{\rightarrow} E \stackrel{p}{\rightarrow} B$ be an étale locally trivial fibration such that the fiber $F$ is a smooth cellular scheme. Assume that this fibration becomes trivial after a finite étale cover of $B$ of degree $d$. Assume furthermore that there are elements $\left\{e_{1}, \ldots, e_{r}\right\}$ in $\mathrm{CH}^{*}(E, 0)$ such that $\left\{f_{1}=i^{*}\left(e_{1}\right), \ldots, f_{r}=i^{*}\left(e_{r}\right)\right\}$ is the basis of $\mathrm{CH}^{*}\left(F_{y}, 0\right)$ for each geometric fiber $F_{y}$ of the fibration. Then the map

$$
\Phi: \mathrm{CH}^{*}(F, 0) \otimes_{\mathbb{Z}} \mathrm{CH}^{*}(B) \rightarrow \mathrm{CH}^{*}(E), \quad \sum_{1 \leq i \leq r} f_{i} \otimes b_{i} \mapsto \sum_{1 \leq i \leq r} p^{*}\left(b_{i}\right) e_{i}
$$

is an isomorphism over $\mathbb{Z}\left[d^{-1}\right]$. In particular, $\mathrm{CH}^{*}(E)\left[d^{-1}\right]$ is a free $\mathrm{CH}^{*}(B)\left[d^{-1}\right]$ module with basis $\left\{e_{1}, \ldots, e_{r}\right\}$.

Proof. Let $B^{\prime} \stackrel{q}{\rightarrow} B$ be a finite étale cover such that $E^{\prime}=E \times{ }_{B} B^{\prime} \stackrel{p^{\prime}}{\rightarrow} B^{\prime}$ is a trivial fibration and let $q^{\prime}: E^{\prime} \rightarrow E$ be the other projection. It follows from our assumption and the isomorphism of $\mathrm{CH}^{*}(F, 0)$ under the field extensions that the set $\left\{e_{i}^{\prime}=q^{*}\left(e_{i}\right)\right\}$ restricts to the basis $\left\{f_{i}\right\}$ of $\mathrm{CH}^{*}\left(F_{y}, 0\right)$ for every fiber $F_{y}$ of the fibration $p^{\prime}$.

Setting $\Phi^{\prime}\left(b^{\prime} \otimes f_{j}\right)=p^{*}\left(b^{\prime}\right) e_{j}^{\prime}$ and using the fact that $q^{* *} \circ p^{*}=p^{*} \circ q^{*}$, $p^{*} \circ q_{*}=q_{*}^{\prime} \circ p^{*}$ and $q_{*} \circ q^{*}=d=q_{*}^{\prime} \circ q^{*}$ (see [Bloch 1986, Exercise 5.8(i)]), one checks that the diagram

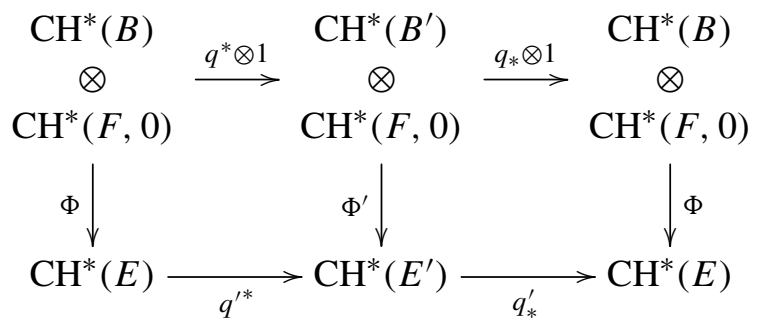

commutes. The middle vertical arrow is an isomorphism by Lemma 6.2. A diagram chase shows that $\Phi$ is an isomorphism over $\mathbb{Z}\left[d^{-1}\right]$.

Theorem 6.5. Let $B$ be a smooth $k$-scheme and let $F \stackrel{i}{\rightarrow} E \stackrel{p}{\rightarrow} B$ be an étale locally trivial fibration such that the fiber $F$ is a smooth cellular scheme. Assume that there are elements $\left\{e_{1}, \ldots, e_{r}\right\}$ in $\mathrm{CH}^{*}(E, 0)$ such that $\left\{f_{1}=i^{*}\left(e_{1}\right), \ldots, f_{r}=i^{*}\left(e_{r}\right)\right\}$ is the basis of $\mathrm{CH}^{*}\left(F_{y}, 0\right)$ for each geometric fiber $F_{y}$ of the fibration. Then the map

$$
\Phi: \mathrm{CH}^{*}(F, 0) \otimes_{\mathbb{Q}} \mathrm{CH}^{*}(B) \rightarrow \mathrm{CH}^{*}(E), \quad \sum_{1 \leq i \leq r} f_{i} \otimes b_{i} \mapsto \sum_{1 \leq i \leq r} p^{*}\left(b_{i}\right) e_{i}
$$

is an isomorphism over the rationals. In particular, $\mathrm{CH}^{*}(E)$ is a free $\mathrm{CH}^{*}(B)$ module with basis $\left\{e_{1}, \ldots, e_{r}\right\}$ over the rationals. 
Proof. We assume all abelian groups to be tensored with $\mathbb{Q}$ in this proof. Since $k$ is perfect and since every étale cover is generically finite, we can find a filtration

$$
\varnothing=B_{n+1} \subsetneq B_{n} \subsetneq \cdots \subsetneq B_{1} \subsetneq B_{0}=B
$$

of $B$ by closed subschemes such that for each $0 \leq i \leq n$, the scheme $V_{i}=B_{i-1} \backslash B_{i}$ is smooth and there is a finite étale cover $V_{i}^{\prime} \rightarrow V_{i}$ such that the given fibration is trivial over $V_{i}^{\prime}$. We set $U_{i}=B \backslash B_{i}$ as before, which implies that $V_{i}=U_{i} \backslash U_{i-1}$. Observe then that each of the $U_{i}$ and $V_{i}$ is smooth. Set $E_{i}=p^{-1}\left(U_{i}\right)$ and $W_{i}=p^{-1}\left(V_{i}\right)$. We prove by induction on $i$ that the map

$$
\mathrm{CH}^{*}(F, 0) \otimes_{\mathbb{Q}} \mathrm{CH}^{*}\left(U_{i}\right) \rightarrow \mathrm{CH}^{*}\left(E_{i}\right)
$$

is an isomorphism, which will imply the proposition. Since $U_{0}=\varnothing$ and since the map $E_{1} \rightarrow U_{1}$ is a smooth fibration which becomes trivial over the finite étale cover $V_{1}^{\prime} \rightarrow V_{1}=U_{1}$, the desired isomorphism for $i=1$ follows from Lemma 6.4. We now consider the diagram

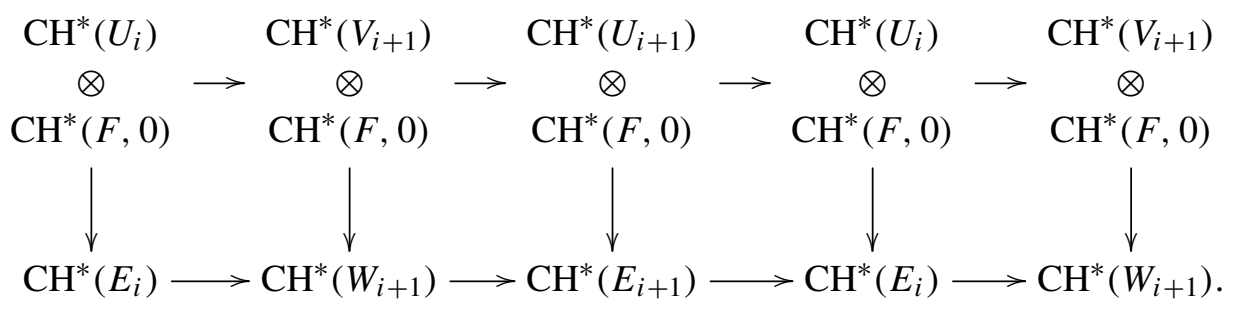

The top row is obtained by tensoring the long exact localization sequence for higher Chow groups with $\mathrm{CH}^{*}(F, 0)$ over $\mathbb{Q}$ and hence is exact. The bottom row is just the localization exact sequence.

One checks as in the proof of Theorem 6.3 that the diagram above is commutative. The first and the fourth vertical arrows are isomorphisms by induction. The second and the fifth vertical arrows are isomorphisms by Lemma 6.4. Hence the middle vertical arrow is also an isomorphism by 5-lemma.

\section{Higher Chow groups of toric bundles and applications}

As an application of Theorem 6.3, we describe the ordinary higher Chow groups of toric bundles with integral coefficients. Let $T$ be a split torus of rank $n$ over $k$ and let $M=\operatorname{Hom}\left(\mathbb{G}_{m}, T\right)$ be the group of its one-parameter subgroups. Let $X=X(\Delta)$ be a smooth projective toric variety associated to a fan $\Delta$ in $M_{\mathbb{R}}$ (see Section 11). Let $B$ be a smooth $k$-scheme and let $p: E \rightarrow B$ be a principal $T$-bundle. Setting $E(X)=E \times{ }^{T} X$, we see that

$$
\pi: E(X) \rightarrow B, \quad \pi((e, x))=p(e)
$$


is a Zariski-locally trivial smooth fibration with all fibers isomorphic to $X$. Since $X$ is projective, it follows that $\pi$ is a projective morphism.

We fix an ordering $\left\{\sigma_{1}, \ldots, \sigma_{m}\right\}$ of $\Delta_{\max }$ and let $\tau_{i} \subset \sigma_{i}$ be the cone that is the intersection of $\sigma_{i}$ with all those $\sigma_{j}$ such that $j \geq i$ and that intersect $\sigma_{i}$ in dimension $n-1$. Let $\tau_{i}^{\prime} \subset \sigma_{i}$ be the cone such that $\tau_{i} \cap \tau_{i}^{\prime}=\{0\}$ and $\operatorname{dim}\left(\tau_{i}\right)+\operatorname{dim}\left(\tau_{i}^{\prime}\right)=n$ for $1 \leq i \leq m$. It is easy to see that $\tau_{i}^{\prime}$ is the intersection of $\sigma_{i}$ with all those $\sigma_{j}$ such that $j \leq i$ and that intersect $\sigma_{i}$ in dimension $n-1$. Since $X$ is smooth and projective, it is well-known that we can choose the ordering above of $\Delta_{\max }$ such that

$$
\tau_{i} \subset \sigma_{j} \text { implies } i \leq j \text { and } \tau_{i}^{\prime} \subset \sigma_{j} \text { implies } j \leq i .
$$

Let $\Delta_{1}=\left\{\rho_{1}, \ldots, \rho_{d}\right\}$ be the set of one-dimensional cones in $\Delta$ and let $\left\{v_{1}, \ldots, v_{d}\right\}$ be the associated primitive elements of $M$. We choose $\left\{\rho_{1}, \ldots, \rho_{n}\right\}$ to be a set of one-dimensional faces of $\sigma_{m}$ such that $\left\{v_{1}, \ldots, v_{n}\right\}$ is a basis of $M$. Let $\left\{\chi_{1}, \ldots, \chi_{n}\right\}$ be the dual basis of $M^{\vee}$.

Definition 7.1. Let $A$ be a commutative ring with unit and let $\left\{r_{1}, \ldots, r_{n}\right\}$ be a subset of $A$. Let $I_{A}$ denote the ideal of the polynomial algebra $A\left[t_{1}, \ldots, t_{d}\right]$ generated by the elements

$$
t_{j_{1}} \cdots t_{j_{l}} \text { for } 1 \leq j_{p} \leq d
$$

such that $\rho_{j_{1}}, \ldots, \rho_{j_{l}}$ do not span a cone of $\Delta$. Let $\bar{I}_{\Delta}$ denote the ideal of $A\left[t_{1}, \ldots, t_{d}\right]$ generated by $I_{\Delta}$ and the relations

$$
s_{i}:=\left(\sum_{j=1}^{d}\left\langle\chi_{i}, v_{j}\right\rangle t_{j}\right)-r_{i} \quad \text { for } 1 \leq i \leq n .
$$

We define the $A$-algebras $R_{\text {eq }}(A, \Delta)$ and $R(A, \Delta)$ to be quotients of $A\left[t_{1}, \ldots, t_{d}\right]$ by the ideals $I_{\Delta}$ and $\bar{I}_{\Delta}$, respectively.

The ring $R_{\mathrm{eq}}(A, \Delta)$ is also known in the literature as the Stanley-Reisner algebra over $A$ associated to the fan $\Delta$; see [Sankaran and Uma 2003, Definition 2.1]. Notice that any character $\chi$ of $T$ acts on $R_{\mathrm{eq}}(A, \Delta)$ through the multiplication by the element $\sum_{j=1}^{d}\left\langle\chi, v_{j}\right\rangle t_{j}$. This makes $R_{\text {eq }}(A, \Delta)$ into an $S=S(T)$-algebra.

Any $T$-equivariant line bundle $L \rightarrow X$ uniquely defines a line bundle

$$
E(L)=E \times{ }^{T} L
$$

on $E(X)$. Every $\rho \in \Delta_{1}$ defines a unique $T$-equivariant line bundle $L_{\rho}$ on $X$ with a $T$-equivariant section $s_{\rho}: X \rightarrow L_{\rho}$ that is transverse to the zero section and whose zero locus is the orbit closure $V_{\rho}=\overline{O_{\rho}}$. For any $\sigma \in \Delta$, let $u_{\sigma}$ denote the fundamental class of the $T$-invariant cycle $\left[V_{\sigma}\right]$ in $\mathrm{CH}^{*}(X, 0)$ and let $y_{\sigma}$ denote the cycle $\left[E\left(V_{\sigma}\right)\right]$ in $\mathrm{CH}^{*}(E(X), 0)$. Notice that $\pi_{\sigma}: E\left(V_{\sigma}\right) \rightarrow B$ is a smooth projective toric subbundle of $\pi: E(X) \rightarrow B$ with fiber $V_{\sigma}$. 
Suppose that $\rho_{j_{1}}, \ldots, \rho_{j_{l}}$ do not span a cone in $\Delta$. Then $s=\left(s_{j_{1}}, \ldots, s_{j_{l}}\right)$ is a nowhere vanishing section of $L_{\rho_{j_{1}}} \oplus \cdots \oplus L_{\rho_{j_{l}}}$ and hence

$$
c_{1}\left(E\left(L_{\rho_{j_{1}}}\right)\right) \cdots c_{1}\left(E\left(L_{\rho_{j_{l}}}\right)\right)=0 \text { in } \mathrm{CH}^{*}(E(X)) .
$$

In particular, we get

$$
y_{\rho_{j_{1}}} \cdots y_{\rho_{j_{l}}}=0 \text { in } \mathrm{CH}^{*}(E(X)) .
$$

We now consider the commutative diagram

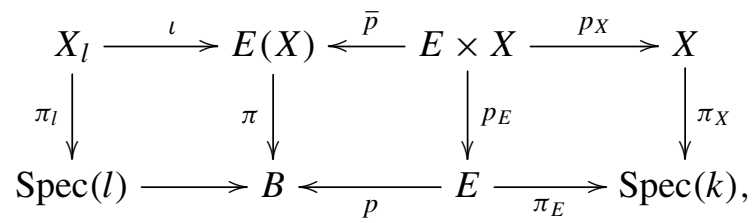

where $\operatorname{Spec}(l)$ is any point of $B$. It is clear that all squares are Cartesian and all the maps in the right square are $T$-equivariant. Let $L_{\chi}$ denote the $T$-equivariant line bundle on $\operatorname{Spec}(k)$ associated to a character $\chi$ of $T$. Since $p$ and $\bar{p}$ are principal $T$-bundles, we see that there is a unique line bundle $\zeta$ on $B$ such that

$$
\pi_{E}^{*}\left(L_{\chi}\right)=p^{*}(\zeta) \quad \text { and } \quad p_{X}^{*} \circ \pi_{X}^{*}\left(L_{\chi}\right)=\bar{p}^{*} \circ \pi^{*}(\zeta)
$$

Using the identity

$$
\pi_{X}^{*}\left(c_{1}^{T}\left(L_{\chi}\right)\right)=\sum_{\rho \in \Delta_{1}}\left\langle\chi, v_{\rho}\right\rangle u_{\rho} \quad \text { in } \mathrm{CH}_{T}^{*}(X)
$$

and the isomorphisms $\mathrm{CH}^{*}(B) \cong \mathrm{CH}_{T}^{*}(E), \mathrm{CH}^{*}(E(X)) \cong \mathrm{CH}_{T}^{*}(E \times X)$, we see that $\pi^{*}\left(c_{1}(\zeta)\right)=\sum_{\rho \in \Delta_{1}}\left\langle\chi, v_{\rho}\right\rangle y_{\rho}$. Let $\zeta_{i} \in \operatorname{Pic}(B)$ be such that $\pi_{E}^{*}\left(L_{\chi_{i}}\right)=p^{*}\left(\zeta_{i}\right)$, where $\left\{\chi_{1}, \ldots, \chi_{n}\right\}$ is a chosen basis of $M^{\vee}$ as above. Setting $r_{i}=c_{1}\left(\zeta_{i}\right) \in \mathrm{CH}^{*}(B)$, we conclude that

$$
\pi^{*}\left(r_{i}\right)=\sum_{j=1}^{d}\left\langle\chi_{i}, v_{j}\right\rangle y_{\rho_{j}} \quad \text { in } \mathrm{CH}^{*}(E(X)), \quad 1 \leq i \leq n .
$$

We define a homomorphism of $\mathrm{CH}^{*}(B)$-algebras

$$
\mathrm{CH}^{*}(B)\left[t_{1}, \ldots, t_{d}\right] \rightarrow \mathrm{CH}^{*}(E(X))
$$

by the assignment $t_{i} \mapsto y_{\rho_{i}}$ for $1 \leq i \leq d$. It follows from (7-4) and (7-7) that this homomorphism descends to a $\mathrm{CH}^{*}(B)$-algebra homomorphism

$$
\psi: R\left(\mathrm{CH}^{*}(B), \Delta\right) \rightarrow \mathrm{CH}^{*}(E(X)) .
$$


The following result describes the higher Chow groups of the projective toric bundle $\pi: E(X) \rightarrow B$ and generalizes [Sankaran and Uma 2003, Theorem 1.2(iii)] to higher Chow groups.

Theorem 7.2. The homomorphism $\psi$ is an isomorphism.

Proof. To prove this theorem, we first observe that for any $\sigma \in \Delta$ and any point $\operatorname{Spec}(l) \rightarrow B$, one has $\iota^{*}\left(E\left(V_{\sigma}\right)\right)=\left(V_{\sigma}\right)_{l}$. Also, it is well-known [Sankaran and Uma 2003, Lemma 3.1] that $\left\{\iota^{*}\left(y_{\tau_{1}}\right), \ldots, \iota^{*}\left(y_{\tau_{m}}\right)\right\}$ forms a $\mathbb{Z}$-basis of $\mathrm{CH}^{*}\left(X_{l}, 0\right)$.

Since $y_{\tau_{i}}=y_{\rho_{i_{1}}} \cdots y_{\rho_{i_{p}}}$ for every $1 \leq i \leq m$, where $\left\{\rho_{i_{1}}, \ldots, \rho_{i_{p}}\right\}$ is the set of edges of $\tau_{i}$, it follows from the proof of Theorem 6.3 that $\mathrm{CH}^{*}(E(X))$ is generated by $\left\{y_{\rho_{1}}, \ldots, y_{\rho_{d}}\right\}$ as a $\mathrm{CH}^{*}(B)$-algebra. In particular, the map $\psi$ is surjective.

To prove injectivity, let $x(\sigma)$ denote the monomial $t_{i_{1}} \cdots t_{i_{p}}$ in $R\left(\mathrm{CH}^{*}(B), \Delta\right)$ such that $\left\{\rho_{i_{1}}, \ldots, \rho_{i_{p}}\right\}$ is the set of edges of $\sigma \in \Delta$. Then by [Sankaran and Uma 2003, Lemma 2.1(ii)], the set $\left\{x\left(\tau_{1}\right), \ldots, x\left(\tau_{m}\right)\right\}$ spans $R\left(\mathrm{CH}^{*}(B), \Delta\right)$ as a $\mathrm{CH}^{*}(B)$-module. Since $\psi\left(x\left(\tau_{i}\right)\right)=y_{\tau_{i}}$ for $1 \leq i \leq m$ and since $\mathrm{CH}^{*}(E(X))$ is a free $\mathrm{CH}^{*}(B)$-module with basis $\left\{y_{\tau_{1}}, \ldots, y_{\tau_{m}}\right\}$ by Theorem 6.3, we conclude that $\psi$ must be injective.

\section{7a. Equivariant and ordinary higher Chow groups of smooth projective toric} varieties. As a consequence of Theorem 7.2, we derive some explicit formulas for the equivariant and ordinary higher Chow groups of smooth projective toric varieties with integral coefficients. We shall later show in Section 11 that such formulas hold for all smooth toric varieties with rational coefficients. Recall that if $X=X(\Delta)$ is a toric variety, then for every $\sigma \in \Delta$, the orbit closure $V_{\sigma}$ is a $T$-invariant closed toric subvariety of $X$ and hence uniquely defines a class $y_{\sigma}=\left[V_{\sigma}\right]$ in $\mathrm{CH}_{T}^{*}(X, 0)$; see [Edidin and Graham 1998, Section 2.2]. This is called the fundamental equivariant class of $V_{\sigma}$.

We consider $A=\mathrm{CH}^{*}(k) \otimes_{\mathbb{Z}} S \cong \mathrm{CH}^{*}(k)\left[t_{1}, \ldots, t_{n}\right]$ as a graded $\mathrm{CH}^{*}(k)$-algebra whose degree zero part is $\mathrm{CH}^{*}(k)$. We have seen above that $R_{\mathrm{eq}}\left(\mathrm{CH}^{*}(k), \Delta\right)$ has an action of $S$ that makes it a graded $A$-algebra. Moreover, $R\left(\mathrm{CH}^{*}(k), \Delta\right)$ is just the quotient $R_{\text {eq }}\left(\mathrm{CH}^{*}(k), \Delta\right) \otimes_{S} \mathbb{Z} \cong R_{\text {eq }}\left(\mathrm{CH}^{*}(k), \Delta\right) \otimes_{A} \mathrm{CH}^{*}(k)$.

Corollary 7.3. Let $X=X(\Delta)$ be a smooth projective toric variety as above. Then the assignment $t_{i} \mapsto y_{\rho_{i}}$ induces $\mathrm{CH}^{*}(k)$-algebra isomorphisms

$$
\begin{aligned}
& \Psi_{X}: R_{\mathrm{eq}}\left(\mathrm{CH}^{*}(k), \Delta\right) \stackrel{\sim}{\rightarrow} \mathrm{CH}_{T}^{*}(X), \\
& \left.\bar{\Psi}_{X}: R\left(\mathrm{CH}^{*}(k), \Delta\right)\right) \stackrel{\sim}{\rightarrow} \mathrm{CH}^{*}(X) .
\end{aligned}
$$

Proof. The second isomorphism is just a special case of Theorem 7.2 when $B=$ $\operatorname{Spec}(k)$.

To prove the isomorphism of (7-9), we first observe that $\Psi_{X}$ is a graded $A$-linear homomorphism; see Section 11. Let $M$ denote the kernel of this map. It follows 
from [Krishna 2009a, Theorem 4.2] that $\mathrm{CH}_{T}^{*}(X)$ is a free $A$-module with basis $\left\{y_{\tau_{i}}\right\}$. It follows from this that $\Psi_{X}$ is surjective and we get an exact sequence of graded $A$-modules

$$
0 \rightarrow M \rightarrow R_{\mathrm{eq}}\left(\mathrm{CH}^{*}(k), \Delta\right) \stackrel{\Psi_{X}}{\longrightarrow} \mathrm{CH}_{T}^{*}(X) \rightarrow 0 .
$$

The freeness of $\mathrm{CH}_{T}^{*}(X)$ as an $A$-module ensures that this sequence remains short exact after tensoring with $\mathrm{CH}^{*}(k)$ via the augmentation $A \rightarrow \mathrm{CH}^{*}(k)$.

It follows from [Krishna 2009a, Theorem 1.1] that

$$
\mathrm{CH}_{T}^{*}(X) \otimes_{A} \mathrm{CH}^{*}(k) \stackrel{\sim}{\longrightarrow} \mathrm{CH}^{*}(X) .
$$

In particular, $\Psi_{X}$ becomes an isomorphism after tensoring with $\mathrm{CH}^{*}(k)$. We conclude that $M \otimes_{A} \mathrm{CH}^{*}(k)=0$. Since $M$ is a nonnegatively graded $A$-module, it must be zero.

\section{Higher Chow groups of flag bundles and applications}

We remind the readers of our convention that all the higher Chow groups for the rest of this text will be considered with rational coefficients. We shall however, indicate the coefficients in the statement of all results.

In this section, we describe a formula for the higher Chow groups of complete flag bundles with rational coefficients. Such a formula for general flag bundles is an immediate consequence of the case of complete flag bundles. We also give some applications of this formula to the theory of equivariant higher Chow groups.

8a. Complete flag bundles. Let $G$ be a connected reductive group over $k$ and let $B$ be a Borel subgroup of $G$ containing a split maximal torus $T$. Let $X$ be a $k$-scheme and let $p: E \rightarrow X$ be a principal $G$-bundle and let $\pi: E / B \rightarrow X$ be the associated complete flag bundle. Vistoli [1989] described the classical Chow groups $\mathrm{CH}_{*}(E, 0)$ and $\mathrm{CH}_{*}(E / B, 0)$ in terms of the Chow groups of $X$. In this section we generalize Vistoli's results to the case of higher Chow groups. The proof below is completely different from Vistoli's; it is much shorter and relies more on the equivariant techniques.

The restriction map $r_{T, X}^{G}$ induces for every $i \geq 0$, a natural map of $S(T)$-modules

$$
\mathrm{CH}_{*}^{G}(E, i) \otimes_{S(G)} S(T) \rightarrow \mathrm{CH}_{*}^{T}(E, i), \quad w \otimes \alpha \mapsto \alpha \cdot r_{T, E}^{G}(w) .
$$

Since $G$ acts freely on $E$, one identifies $\mathrm{CH}_{*}^{G}(E, i)$ with $\mathrm{CH}_{*}(X, i)$ by Proposition 2.2. The group $\mathrm{CH}_{*}^{B}(E, i)$ is canonically identified with $\mathrm{CH}_{*}(E / B, i)$ for the same reason. The map above then translates into a natural map of $S(T)$-modules

$$
\lambda_{X}: \mathrm{CH}_{*}(X, i) \otimes_{S(G)} S(T) \rightarrow \mathrm{CH}_{*}(E / B, i) .
$$


Taking the direct sum over $\left\{\mathrm{CH}_{*}(X, i)\right\}_{i \geq 0}$, we get a natural map of $S(T)$-modules

$$
\lambda_{X}: \mathrm{CH}_{*}(X) \otimes_{S(G)} S(T) \rightarrow \mathrm{CH}_{*}(E / B) .
$$

This map is a ring homomorphism if $X$ is smooth. One can easily check that $\lambda_{X}$ commutes with the flat pull-back and the proper push-forward maps between the higher Chow groups of the base schemes of the bundle. We wish to show that $\lambda_{X}$ is an isomorphism. We begin with the following special case.

Lemma 8.1. Let $X$ be a smooth (not necessarily connected) scheme over $k$ and let $f: X^{\prime} \rightarrow X$ be a finite étale morphism such that the principal bundle $p: E \rightarrow X$ is trivialized over $X^{\prime}$. Then the map $\lambda_{X}$ is an isomorphism with rational coefficients. In particular, $\lambda_{X}$ is an isomorphism with rational coefficients if $X$ is zero-dimensional.

Proof. Since $X$ is a disjoint union of connected smooth schemes, it is enough to prove the lemma when $X$ is smooth and connected. If $E / B=G / B \rightarrow \operatorname{Spec}(k)$ is the flag variety, then we have

$$
\begin{aligned}
\mathrm{CH}_{*}(k) \otimes_{S(G)} S(T) & \cong \mathrm{CH}_{*}(k) \otimes_{\mathrm{CH}^{*}(k, 0)}\left(\mathrm{CH}^{*}(k, 0) \otimes_{S(G)} S(T)\right) \\
& \cong \mathrm{CH}_{*}(k) \otimes_{\mathrm{CH}^{*}(k, 0)} \mathrm{CH}_{*}(G / B, 0) \\
& \cong \mathrm{CH}_{*}(G / B) \quad \text { (by Lemma 6.2), }
\end{aligned}
$$

where $\cong^{\dagger}$ follows from [Demazure 1973, théorème 2].

If $G / B \times X \stackrel{\pi}{\rightarrow} X$ is the trivial bundle, then we get

$$
\begin{aligned}
\mathrm{CH}_{*}(X) \otimes_{S(G)} S(T) & \cong \mathrm{CH}_{*}(X) \otimes_{\mathrm{CH}^{*}(k, 0)}\left(\mathrm{CH}^{*}(k, 0) \otimes_{S(G)} S(T)\right) \\
& \cong \mathrm{CH}_{*}(X) \otimes_{\mathrm{CH}^{*}(k, 0)} \mathrm{CH}_{*}(G / B, 0) \\
& \cong \mathrm{CH}_{*}(G / B \times X) \quad \text { (by Lemma 6.2). }
\end{aligned}
$$

In general, we consider the diagram

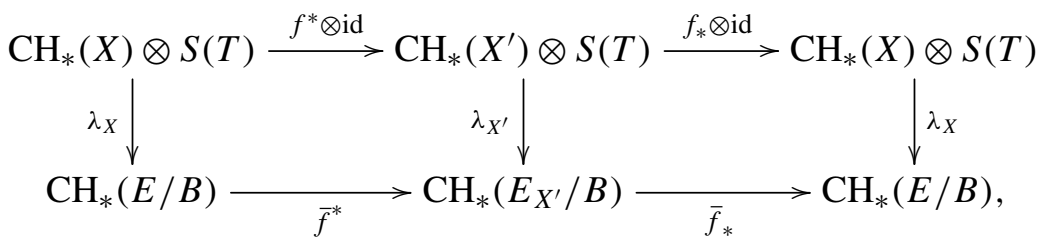

where the tensor product in the top row is over $S(G)$. We have just shown that $\lambda_{X^{\prime}}$ is an isomorphism. It follows from the projection formula (see [Bloch 1986, Exercise 5.8]) that the composite horizontal maps in both rows are multiplication by $\left[k\left(X^{\prime}\right): k(X)\right]$. Hence, $\lambda_{X}$ must be an isomorphism too.

Theorem 8.2. For any $k$-scheme $X$, the map $\lambda_{X}$ is an isomorphism of $S(T)$-modules with rational coefficients. This is a ring isomorphism if $X$ is smooth. 
Proof. We only need to prove the first assertion, for which we use induction on the dimension of $X$. The zero-dimensional case follows from Lemma 8.1. In general, we can find an étale cover of $X$ over which the bundle $p: E \rightarrow X$ becomes trivial. Since any such cover is generically finite and since the base field $k$ is perfect, we can find a dense open subset $j: U \hookrightarrow X$ and a finite étale cover $f: U^{\prime} \rightarrow U$ such that $U$ is a disjoint union of connected smooth schemes and the given bundle is trivial over $U^{\prime}$. Let $\iota: Z \hookrightarrow X$ be the complement of $U$ with its reduced induced closed subscheme structure.

We now consider the diagram

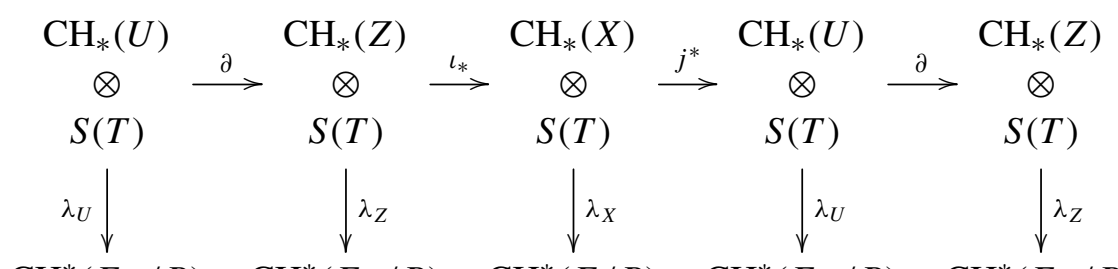

$\mathrm{CH}^{*}\left(E_{U} / B\right) \rightarrow \mathrm{CH}^{*}\left(E_{Z} / B\right) \rightarrow \mathrm{CH}^{*}(E / B) \rightarrow \mathrm{CH}^{*}\left(E_{U} / B\right) \rightarrow \mathrm{CH}^{*}\left(E_{Z} / B\right)$

of localization exact sequences, where the tensor product in the top row is over the ring $S(G)$. In particular, this row is exact by the flatness of $S(T)$ over $S(G)$. The second and the third squares commute by the compatibility of $\lambda_{X}$ with the push-forward and the pull-back maps as remarked above.

To see that the first square commutes, let us consider an element

$$
a \otimes b \in \mathrm{CH}_{*}(U) \otimes S(T) .
$$

If we map this horizontally, we get $\partial(a) \otimes b$, which is mapped vertically down to $b \cdot \pi_{Z}^{*} \circ \partial(a)$. Since the localization sequence of higher Chow groups is compatible with respect to the flat pull-back, this last term is same as $b \cdot \partial \circ \pi_{U}^{*}(a)$. On the other hand, mapping $a \otimes b$ vertically down gives $b \cdot \pi_{U}^{*}(a)$ and if we map this horizontally, we get $\partial\left(b \cdot \pi_{U}^{*}(a)\right)$. Since the horizontal maps in the bottom row are $S(T)$-linear (see Proposition 2.2), we conclude that the first (hence the fourth) square commutes.

The first and the fourth vertical arrows in the diagram above are isomorphisms by Lemma 8.1 . Since $U$ is a dense open in $X$, the dimension of $Z$ is strictly smaller than that of $X$. Hence the second and the fifth vertical arrows are isomorphisms by induction on the dimension and Lemma 8.1. We conclude from the 5-lemma that $\lambda_{X}$ is an isomorphism.

8b. Principal bundles and flag bundles. The following extension of Theorem 8.2 to all flag bundles is a direct generalization of the projective bundle formula for higher Chow groups.

Corollary 8.3. Let $p: E \rightarrow X$ be a principal $G$-bundle over a $k$-scheme $X$ and let $\pi: E / P \rightarrow X$ denote the flag bundle associated to a parabolic subgroup $P$. Then 
the natural map of $S(P)$-modules

$$
\lambda_{X}: \mathrm{CH}_{*}(X ; \mathbb{Q}) \underset{S(G ; \mathbb{Q})}{\otimes} S(P ; \mathbb{Q}) \rightarrow \mathrm{CH}_{*}(E / P ; \mathbb{Q}), \quad w \otimes \alpha \mapsto \alpha \cdot r_{P, E}^{G}(w) .
$$

is an isomorphism. This is a ring isomorphism if $X$ is smooth.

Proof. We only need to prove the first assertion. Let $B \subseteq P$ be a Borel subgroup of $G$ containing a split maximal torus $T$. Let $\Phi(G, T)$ be the root system of $G$ with respect to $T$ such that $B$ corresponds to the base $\Delta$ of $\Phi(G, T)$ and $P$ corresponds to a subset $I \subset \Delta$. Let $P=M \ltimes N$ be the Levi decomposition and let $B_{M}=B \cap M$ be the Borel subgroup of $M$ containing $T$. Let $W_{P} \subset W$ be the Weyl group of $P$ with respect to $T$. It follows from Propositions 3.3, 3.4 and Corollary 8.7 that the natural map

$$
\mathrm{CH}_{*}(E / P) \rightarrow\left(\mathrm{CH}_{*}(E / B)\right)^{W_{P}}
$$

is an isomorphism.

On the other hand, following the proof of Corollary 8.7, we get

$$
\begin{aligned}
\left(\mathrm{CH}_{*}(X) \otimes_{S(G)} S(T)\right)^{W_{P}} & \cong \mathrm{CH}_{*}(X) \otimes_{S(G)}(S(T))^{W_{P}} \\
& \cong \mathrm{CH}_{*}(X) \otimes_{S(G)} S(M) \\
& \cong \mathrm{CH}_{*}(X) \otimes_{S(G)} S(P),
\end{aligned}
$$

where the last isomorphism follows again from Proposition 3.4. The corollary now follows from Theorem 8.2 by combining (8-3) and (8-4).

The following result generalizes [Vistoli 1989, Corollary 3.2] to higher Chow groups.

Corollary 8.4. Let $G$ be connected and split reductive group over $k$ and let $p: E \rightarrow X$ be a principal $G$-bundle over a $k$-scheme $X$. Then there is a strongly convergent spectral sequence

$$
E_{2}^{p, q}=\operatorname{Tor}_{p}^{S(G ; \mathbb{Q})}\left(\mathbb{Q}, \mathrm{CH}_{*}(X, q ; \mathbb{Q})\right) \Rightarrow \mathrm{CH}_{*}(E, p+q ; \mathbb{Q}) .
$$

The edge homomorphism yields an isomorphism

$$
\mathrm{CH}_{*}(X, 0 ; \mathbb{Q}) \underset{S(G ; \mathbb{Q})}{\otimes} \mathbb{Q} \stackrel{\sim}{\longrightarrow} \mathrm{CH}_{*}(E, 0 ; \mathbb{Q}) .
$$

Proof. This is an immediate consequence of Theorem 8.2, [Krishna 2009a, Theorem 1.1] and flatness of $S(T)$ over $S(G)$.

8c. A change of groups isomorphism. The following theorem is an analogue of a similar result in equivariant $K$-theory by Merkurjev [2005, Proposition 8]. However, this result for the equivariant higher Chow groups has an advantage over Merkurjev's theorem in that it holds for the action of any split reductive group (though with 
rational coefficients) whereas [ibid., Proposition 8] is known only for the groups whose derived subgroups are simply connected, for example, $\mathrm{GL}_{n}$. The special case $\mathrm{CH}_{*}^{G}(X, 0)$ of the result below was proven by Brion [1997, Theorem 6.7].

Theorem 8.5. Let $G$ be a connected reductive group and let $T$ be a split maximal torus of $G$. Then for any $X \in \mathscr{V}_{G}$, the natural map of $S(T)$-modules

$$
\lambda_{X}: \mathrm{CH}_{*}^{G}(X ; \mathbb{Q}) \underset{S(G ; \mathbb{Q})}{\otimes} S(T ; \mathbb{Q}) \rightarrow \mathrm{CH}_{*}^{T}(X ; \mathbb{Q})
$$

is an isomorphism. This is a ring isomorphism if $X$ is smooth.

Proof. We only need to show the first assertion. If $(V, U)$ is a good pair for the $G$-action, then $\mathrm{CH}_{*}^{G}(X, i)$ and $\mathrm{CH}_{*}^{T}(X, i)$ in suitable degrees are the same as $\mathrm{CH}_{*}\left(X_{G}, i\right)$ and $\mathrm{CH}_{*}\left(X_{B}, i\right)$, respectively, where $B$ is a Borel subgroup of $G$ containing $T$. Hence, it suffices to show that for any $k$-scheme $Z$ with a free action of $G$ with quotients $G / B$ and $Z / G$, the natural map

$$
\lambda_{Z / G}: \mathrm{CH}_{*}(Z / G) \otimes_{S(G)} S(T) \rightarrow \mathrm{CH}_{*}(Z / B)
$$

is an isomorphism. But this follows immediately by applying Theorem 8.2 to the principal bundle $Z \rightarrow Z / G$.

Remark 8.6. The first remark is that Theorem 8.5 holds if $G$ is any connected linear algebraic group (not necessarily reductive) if the base field is of characteristic zero. This is an immediate consequence of Proposition 3.4. The second remark is that the theorem above can also be proven as a simple consequence of the Leray-Hirsch theorem 6.5. The case of smooth schemes is a direct consequence of Theorem 6.5 and the general case can be proven using noetherian induction and the localization sequence. We leave it an exercise to fill in the details.

8d. Some consequences of Theorem 8.5. Recall that if $G$ is a connected reductive group with a split maximal torus $T$, then the normalizer $N$ of $T$ in $G$ and all its connected components are defined over $k$ and the quotient $N / T$ is the Weyl group $W$ of the corresponding root system. In particular, $W \subset G / T$. If $G$ acts on a variety $X$ and if $(V, U)$ is a good pair for the $G$-action, then $X \times{ }^{T} U \rightarrow X \times{ }^{G} U$ is an étale-locally trivial smooth fibration with fiber $G / T$. In particular, $W$ acts on each $\mathrm{CH}_{j}^{T}(X, i)$ and the map $\mathrm{CH}_{j}^{G}(X, i) \rightarrow \mathrm{CH}_{j}^{T}(X, i)$ factors through the $W$-invariants. We get the following consequence of Theorem 8.5.

Corollary 8.7 (see Theorem 5.7). Let $G$ be a connected reductive group and let $T$ be a split maximal torus of $G$ with the Weyl group $W$. Then for any $X \in \mathscr{V}_{G}$, the restriction map $r_{T, X}^{G}$ induces an isomorphism

$$
\mathrm{CH}_{*}^{G}(X ; \mathbb{Q}) \stackrel{\sim}{\longrightarrow}\left(\mathrm{CH}_{*}^{T}(X ; \mathbb{Q})\right)^{W} .
$$


Proof. Since $W$ is a finite group, the trivial $\mathbb{Q}[W]$-module $\mathbb{Q}$ is a projective $\mathbb{Q}[W]$-module of finite rank. In particular, it follows from Theorem 8.5 that

$$
\begin{aligned}
\left(\mathrm{CH}_{T}^{*}(X)\right)^{W} & =\operatorname{Hom}_{\mathbb{Q}[W]}\left(\mathbb{Q}, \mathrm{CH}_{*}^{G}(X) \otimes_{S(G)} S(T)\right) \\
& =\mathrm{CH}_{*}^{G}(X) \otimes_{S(G)} \operatorname{Hom}_{\mathbb{Q}[W]}(\mathbb{Q}, S(T)) \\
& =\mathrm{CH}_{*}^{G}(X) \otimes_{S(G)}(S(T))^{W} \\
& ={ }^{\dagger} \mathrm{CH}_{*}^{G}(X) \otimes_{S(G)} S(G) \\
& =\mathrm{CH}_{*}^{G}(X),
\end{aligned}
$$

where $={ }^{\dagger}$ holds by [Edidin and Graham 1998, Proposition 6].

As an important consequence of the result above, we get the following analogue of a similar result of Thomason [1988, Theorem 1.13] in equivariant $K$-theory.

Corollary 8.8 (see Remark 5.8). Let $G$ be a connected and reductive group over $k$ and let $T$ be a split maximal torus in $G$. Then the restriction map

$$
\mathrm{CH}_{*}^{G}(X ; \mathbb{Q}) \stackrel{r_{T, X}^{G}}{\longrightarrow} \mathrm{CH}_{*}^{T}(X ; \mathbb{Q})
$$

is a split monomorphism. Moreover, this splitting is natural for morphisms in $\mathscr{V}_{G}$. In particular, if $H$ is any closed subgroup of $G$, then there is a split injective map

$$
\mathrm{CH}_{*}^{H}(X ; \mathbb{Q}) \stackrel{r_{T, X}^{G}}{\longrightarrow} \mathrm{CH}_{*}^{T}\left(G \times{ }^{H} X ; \mathbb{Q}\right) .
$$

Proof. The first statement follows directly from Corollary 8.7, where the splitting is given by the trace map into the subgroup of $W$-invariants. The last statement follows from (8-6) and Corollary 3.2.

Remark 8.9. Let $\varrho \in S(T) \cong \mathrm{CH}_{G}^{*}(G / B, 0)$ be such that the forgetful map takes $\varrho$ to the class of the zero-dimensional Schubert cycle in $\mathrm{CH}^{*}(G / B, 0)$. For a flag bundle $\pi: E / B \rightarrow X$ over a scheme $X$, if we define $\psi_{X}: \mathrm{CH}_{*}(X) \rightarrow \mathrm{CH}_{*}(X)$ by $\psi_{X}(\alpha)=\pi_{*}\left(\varrho \cdot \pi^{*}(\alpha)\right)$, then it can be shown that $\psi_{X}$ is an isomorphism. In particular, $\pi^{*}$ is split injective. This gives another (and more conceptual) proof of Corollary 8.8. In fact, this proof shows that $r_{T, X}^{G}$ is split injective with integer coefficients if $G$ is special.

Let $G$ be a connected reductive group and let $B$ be a Borel subgroup of $G$ containing a split maximal torus $T$. It follows from [Demazure 1973, théorème 2] that the forgetful map $S(T) \rightarrow \mathrm{CH}^{*}(G / B, 0)$ is surjective. Moreover, if $\left\{\varrho_{w}\right\}_{w \in W}$ are polynomials in $S(T)$ which map to the classes of Schubert cycles $\left\{\zeta_{w}\right\}_{w \in W}$ in $\mathrm{CH}^{*}(G / B, 0)$, then $S(T)$ is a free $S(G)$-module with basis $\left\{\varrho_{w}\right\}_{w \in W}$. The following is a direct generalization of Demazure's theorem to the case of all smooth schemes and all higher Chow groups. This also strengthens Corollary 8.7 for smooth schemes. 
Corollary 8.10. Let $X \in \mathscr{V}_{G}^{S}$ and let $X \stackrel{p_{X}}{\longrightarrow} \operatorname{Spec}(k)$ be the structure map. Set $\varrho_{w, X}=$ $p_{X}^{*}\left(\varrho_{w}\right)$. Then $\mathrm{CH}_{T}^{*}(X ; \mathbb{Q})$ is a free $\mathrm{CH}_{G}^{*}(X ; \mathbb{Q})$-module with basis $\left\{\varrho_{w, X}\right\}_{w \in W}$.

Proof. It follows from the construction that the map

$$
\lambda_{X}: \mathrm{CH}_{G}^{*}(X) \otimes_{S(G)} S(T) \rightarrow \mathrm{CH}_{T}^{*}(X)
$$

takes $1 \otimes \varrho_{w}$ onto $\varrho_{w, X}$. The corollary is now an immediate consequence of Theorem 8.5.

\section{Cohomological rigidity and specializations}

Let $G$ be a split diagonalizable group over $k$ acting on a smooth scheme $X$. Recall [Springer 1998, 13.2.5] that all the diagonalizable subgroups of $G$ are defined and split over $k$. The equivariant $K$-theory of $X$ for the $G$-action was studied by Vezzosi and Vistoli [2003]. Their main result (Theorem 1) is to reconstruct the equivariant $K$-theory ring of $X$ in terms of the equivariant $K$-theory of the loci where the stabilizers have constant dimension. In the next two sections, we use the ideas of Vezzosi and Vistoli to prove an analogous decomposition theorem (see Theorem 10.3) for the equivariant higher Chow groups of $X$ for the $G$-action. As mentioned in the introduction, this theorem and its compatibility with the corresponding result for the equivariant $K$-theory play fundamental roles in the proof of the equivariant Riemann-Roch theorems in [Krishna 2009b]. This theorem is very useful in computing the equivariant and ordinary higher Chow groups of smooth schemes with torus action. Some applications of this kind are given in Section 11.

This section is concerned with the study of the notion of cohomological rigidity and the construction of certain specialization maps in equivariant higher Chow groups. In this and the next section, the group $G$ will denote a split diagonalizable group and the all schemes will be assumed to be smooth with $G$-action. We have seen (Proposition 2.2) that for such a scheme $X, \mathrm{CH}_{G}^{*}(X)$ is a bigraded ring, which is an algebra over the ring $\mathrm{CH}_{G}^{*}(k)$.

\section{9a. Cohomological rigidity.}

Definition 9.1. Let $Y \subset X$ be a smooth and $G$-invariant closed subscheme of codimension $d \geq 0$ and let $N_{Y / X}$ denote the normal bundle of $Y$ in $X$. We say that $Y$ is cohomologically rigid inside $X$ if $c_{d}^{G}\left(N_{Y / X}\right)$ is a not a zero-divisor in the ring $\mathrm{CH}_{G}^{*}(Y)$.

As one observes, this definition has reasonable meaning only in the equivariant setting, since every element of positive degree in the nonequivariant Chow ring is nilpotent. The importance of cohomological rigidity for the equivariant higher 
Chow groups comes from the following analogue of the $K$-theory splitting theorem (Proposition 4.3) of [Vezzosi and Vistoli 2003].

Proposition 9.2. Let $Y$ be a smooth and $G$-invariant closed subscheme of $X$ of codimension $d \geq 0$. Assume that $Y$ is cohomologically rigid inside $X$, and put $U=X \backslash Y$. Let $i: Y \hookrightarrow X$ and $j: U \hookrightarrow X$ be the inclusion maps.

(i) The localization sequence

$$
0 \rightarrow \mathrm{CH}_{G}^{*}(Y ; \mathbb{Q}) \stackrel{i_{*}}{\rightarrow} \mathrm{CH}_{G}^{*}(X ; \mathbb{Q}) \stackrel{j^{*}}{\rightarrow} \mathrm{CH}_{G}^{*}(U ; \mathbb{Q}) \rightarrow 0
$$

is exact.

(ii) The restriction ring homomorphisms

$$
\mathrm{CH}_{G}^{*}(X ; \mathbb{Q}) \stackrel{\left(i^{*}, j^{*}\right)}{\longrightarrow} \mathrm{CH}_{G}^{*}(Y ; \mathbb{Q}) \times \mathrm{CH}_{G}^{*}(U ; \mathbb{Q})
$$

give an isomorphism of rings

$$
\mathrm{CH}_{G}^{*}(X ; \mathbb{Q}) \stackrel{\sim}{\longrightarrow} \mathrm{CH}_{G}^{*}(Y ; \mathbb{Q}) \underset{\mathrm{CH}_{G}^{*}(Y ; \mathbb{Q})}{\times} \mathrm{CH}_{G}^{*}(U ; \mathbb{Q}),
$$

where $\widetilde{\mathrm{CH}_{G}^{*}(Y ; \mathbb{Q})}=\mathrm{CH}_{G}^{*}(Y ; \mathbb{Q}) /\left(c_{d}^{G}\left(N_{Y / X}\right)\right)$, and the maps

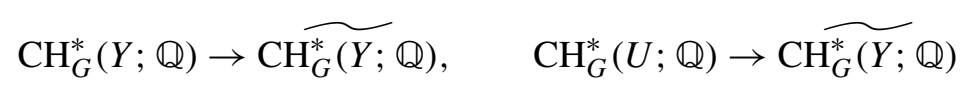

are, respectively, the natural surjection and the map

$$
\mathrm{CH}_{G}^{*}(U ; \mathbb{Q})=\frac{\mathrm{CH}_{G}^{*}(X ; \mathbb{Q})}{i_{*}\left(\mathrm{CH}_{G}^{*}(Y ; \mathbb{Q})\right)} \stackrel{i^{*}}{\rightarrow} \frac{\mathrm{CH}_{G}^{*}(Y ; \mathbb{Q})}{c_{d}^{G}\left(N_{Y / X}\right)}=\widetilde{\mathrm{CH}_{G}^{*}(Y ; \mathbb{Q}),}
$$

which is well-defined by Corollary 4.5.

Proof. Part (i) follows directly from Corollary 4.5 and the definition of cohomological rigidity. Since $i^{*}$ and $j^{*}$ are ring homomorphisms, the proof of the second part follows directly from the first part and [Vezzosi and Vistoli 2003, Lemma 4.4].

To apply the result above in our context, we need to find some sufficient conditions for checking the cohomological rigidity in specific examples. We begin with the following elementary result.

Lemma 9.3. Let $A$ be a ring which is a $\mathbb{Q}$-algebra. Then an element of the form $t^{d}$, where $t=\sum_{j=1}^{r} a_{j} t_{i_{j}} \in A\left[t_{1}, \ldots, t_{n}\right]$, is not a zero-divisor for any $d \geq 0$ whenever $a_{j} \in \mathbb{Q}$ for all $j$ and $a_{j} \neq 0$ for some $j$.

Proof. Since all $a_{j} \in \mathbb{Q}$ and some $a_{j} \neq 0$, we see that $t^{d}$ is a nonzero element of $\mathbb{Q}\left[t_{1}, \ldots, t_{n}\right]$ and hence a nonzero divisor in this ring. Since tensoring with $A$ over $\mathbb{Q}$ is exact, we see that the multiplication by $t^{d}$ is injective in $A\left[t_{1}, \ldots, t_{n}\right]$ too. 
Proposition 9.4. Let $G$ be a split diagonalizable group acting on a smooth scheme $X$ and let $E$ be a $G$-equivariant vector bundle of rank $d$ on $X$. Assume that there is a subtorus $T \subset G$ of positive rank which acts trivially on $X$, such that in the eigenspace decomposition of $E$ with respect to $T$, the submodule corresponding to the trivial character is zero. Then $c_{d}^{G}(E)$ is not a zero-divisor in $\mathrm{CH}_{G}^{*}(X ; \mathbb{Q})$.

Proof. By [Thomason 1986, Lemma 5.6], E has a unique direct sum decomposition

$$
E=\bigoplus_{i=1}^{r} E_{\chi_{i}} \otimes L_{\chi_{i}},
$$

where we choose a splitting $G=D \times T, E_{\chi_{i}}$ are $D$-bundles and $\chi_{i}$ are characters of $T$ with associated line bundles $L_{\chi_{i}} \in \operatorname{Pic}_{T}(k)$. This decomposition is via the functor

$$
\operatorname{Bun}_{X}^{D} \times \operatorname{Rep}(T) \rightarrow \operatorname{Bun}_{X}^{G}, \quad(F, \rho) \mapsto p_{1}^{*}(F) \otimes p_{2}^{*}(\rho),
$$

where $p_{1}: D \times T \rightarrow D$ and $p_{2}: D \times T \rightarrow T$ are the projections.

Since $\operatorname{rank}(E)=d$, the Whitney sum formula yields $c_{d}^{G}(E)=\prod_{i=1}^{r} c_{d_{i}}^{G}\left(E_{\chi_{i}} \otimes L_{\chi_{i}}\right)$, where $d_{i}=\operatorname{rank}\left(E_{\chi_{i}}\right)$. We can thus assume that $E=E_{\chi} \otimes L_{\chi}$, where $\chi$ is not a trivial character by our assumption. In particular, we can write

$$
c_{1}^{T}\left(L_{\chi}\right)=t=\sum_{i=1}^{p} n_{i} t_{i} \in \mathbb{Q}\left[t_{1}, \ldots, t_{n}\right]
$$

with $n_{i} \neq 0$ for some $i$. By neglecting those $i$ for which the coefficients $n_{i}$ are zero, we can assume that $n_{i} \neq 0$ for all $i$. Now we have

$$
\begin{aligned}
c_{d}^{G}(E) & =c_{d}^{G}\left(p_{1}^{*}\left(E_{\chi}\right) \otimes p_{2}^{*}\left(L_{\chi}\right)\right)=^{\dagger} \sum_{i=0}^{d} c_{d-i}^{G}\left(p_{1}^{*}\left(E_{\chi}\right)\right) \cdot\left(c_{1}^{G}\left(p_{2}^{*}\left(L_{\chi}\right)\right)\right)^{i} \\
& =\sum_{i=0}^{d} p_{1}^{*}\left(c_{d-i}^{D}\left(E_{\chi}\right)\right) \cdot p_{2}^{*}\left(\left(c_{1}^{T}\left(L_{\chi}\right)\right)^{i}\right)=\sum_{i=0}^{d} \alpha_{i} t^{i},
\end{aligned}
$$

where $\alpha_{i} \in \mathrm{CH}_{D}^{*}(X)$ and $c_{d}^{G}(E) \in \mathrm{CH}_{G}^{*}(X) \cong \mathrm{CH}_{D}^{*}(X) \otimes S(T)$ by Theorem 3.5 and $={ }^{\dagger}$ holds by [Fulton 1984, Remark 3.2.3]. Furthermore, $\alpha_{d}=p_{1}^{*}\left(c_{0}^{D}\left(E_{\chi}\right)\right)=1$. Thus we get $c_{d}^{G}(E)=t^{d}+\alpha_{d-1} t^{d-1}+\cdots+\alpha_{1} t+\alpha_{0}=g(t)$.

We need to show that $g(t)$ is not a zero divisor in $\mathrm{CH}_{D}^{*}(X)\left[t_{1}, \ldots, t_{n}\right]$. So suppose $f(\underline{t})$ is a nonzero polynomial such that $g(t) f(\underline{t})=0$, and let $f^{\prime}(\underline{t})$ be the homogeneous part of $f(\underline{t})$ of largest degree which is not zero. By comparing the homogeneous parts, it is easy to see that $g(t) f(\underline{t})=0$ only if $t^{d} f^{\prime}(\underline{t})=0$. But this is a contradiction since $t$ satisfies the condition of Lemma 9.3 by (9-1), and hence is not a zero-divisor. 
Let $G$ be a split diagonalizable group as above and let $X \in \mathscr{V}_{G}^{S}$. Following the notation of [Vezzosi and Vistoli 2003], for any $s \geq 0$, we let $X_{\leq s} \subset X$ be the open subset of points whose stabilizers have dimension at most $s$. We shall often write $X_{\leq s-1}$ also as $X_{<s}$. Let $X_{s}=X_{\leq s} \backslash X_{<s}$ denote the locally closed subset of $X$, where the stabilizers have dimension exactly $s$. We think of $X_{S}$ as a subspace of $X$ with the reduced induced structure. It is clear that $X_{\leq s}$ and $X_{s}$ are $G$-invariant subspaces of $X$. Let $N_{s}$ denote the normal bundle of $X_{s}$ in $X_{\leq s}$, and let $N_{s}^{0}$ denote the complement of the 0 -section in $N_{s}$. Then $G$ clearly acts on $N_{s}$. The following result describes some very useful properties of these subspaces.

Proposition 9.5. Let $s \geq 0$ be an integer.

(i) There exists a finite number of s-dimensional subtori $T_{1}, \ldots, T_{r}$ in $G$ such that $X_{s}$ is the disjoint union of the fixed point spaces $X_{\leq s}^{T_{j}}$.

(ii) $X_{s}$ is smooth locally closed subscheme of $X$.

(iii) $N_{s}^{0}=\left(N_{s}\right)_{<s}$.

Proof. Since the base field $k$ is perfect, this is a special case of [Vezzosi and Vistoli 2003, Proposition 2.2], which holds for regular $G$-schemes over any connected and separated Noetherian base scheme.

Remark 9.6. We mention here that although the proposition above has been stated for the smooth schemes, part (i) of the proposition holds also when $X$ is not necessarily smooth, since the proof given in [loc. cit.] only uses Thomason's generic étale slice theorem, which holds very generally.

Corollary 9.7. For $s \geq 1, X_{s}$ is cohomologically rigid inside $X_{\leq s}$.

Proof. Let $d_{s}$ be the codimension of $X_{s}$ in $X_{\leq s}$. We need to show that $c_{d_{s}}^{G}\left(N_{s}\right)$ is not a zero-divisor in $\mathrm{CH}_{G}^{*}\left(X_{s}\right)$. By Proposition 9.4, it suffices to show that there exists a subtorus $T$ in $G$ of positive rank that acts trivially on $X_{s}$, such that in the eigenspace decomposition of $N_{s}$ with respect to $T$, the submodule corresponding to the trivial character is zero. But this follows directly from parts (i) and (iii) of Proposition 9.5 and the fact that $s \geq 1$; see [Vezzosi and Vistoli 2003, Proposition 4.6].

9b. Specialization maps. Let $G$ and $X$ be as above and let $n$ be the dimension of $G$. As seen above, there is a filtration of $X$ by $G$-invariant open subsets

$$
\varnothing=X_{\leq-1} \subset X_{\leq 0} \subset \cdots \subset X_{\leq n}=X .
$$

In particular, $G$ acts on $X_{\leq 0}$ with finite stabilizers, and the toral component of $G$ acts trivially on $X_{n}$. We fix $1 \leq s \leq n$ and let $f_{s}: X_{s} \hookrightarrow X_{\leq s}$ and $g_{s}: X_{<s} \hookrightarrow X_{\leq s}$ denote the closed and the open embeddings, respectively. Let $\pi: M_{s} \rightarrow \mathbb{P}^{1}$ be the deformation to the normal cone for the embedding $f_{s}$ as in Section 4. We have already observed there that for the trivial action of $G$ on $\mathbb{P}^{1}, M_{s}$ has a 
natural $G$-action. Moreover, the deformation diagram (4-1) is a diagram of smooth $G$-spaces. For $0 \leq t \leq s$, we shall often denote the open subspace $\left(M_{s}\right)_{\leq t}$ of $M_{s}$ by $M_{s, \leq t}$. The terms like $M_{s, t}$ and $M_{s,<t}$ (and also for $N_{s}$ ) will have similar meaning in what follows. Since $G$ acts trivially on $\mathbb{P}^{1}$, it acts on $M_{s}$ fiberwise with $N_{s}=\pi^{-1}(\infty)$ and

$$
M_{s, \leq t} \cap \pi^{-1}\left(\mathbb{A}^{1}\right)=X_{\leq t} \times \mathbb{A}^{1}, \quad M_{s, t} \cap \pi^{-1}\left(\mathbb{A}^{1}\right)=X_{t} \times \mathbb{A}^{1} .
$$

Let $i_{s, \leq t}: N_{s, \leq t} \hookrightarrow M_{s, \leq t}$ and $j_{s, \leq t}: X_{\leq t} \times \mathbb{A}^{1} \hookrightarrow M_{s, \leq t}$ denote the obvious closed and open embeddings. We define $i_{s, t}$ and $j_{s, t}$ similarly. Let $\eta_{s, t}: N_{s, t} \hookrightarrow N_{s, \leq t}$ and $\delta_{s, t}: M_{s, t} \hookrightarrow M_{s, \leq t}$ denote the other closed embeddings. One has a commutative diagram

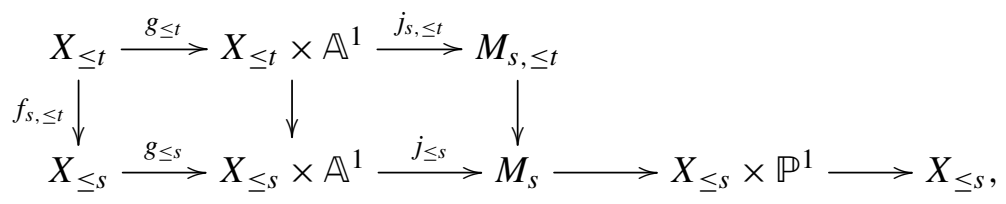

where $g_{\leq t}$ is the 0 -section embedding, and the composite of all the maps in the bottom row is identity. This gives us the diagram

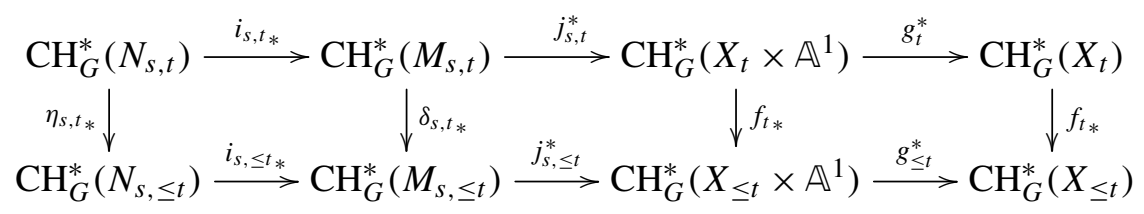

of equivariant higher Chow groups, where the left square commutes by the covariance of the push-forward map, the middle commutes by Proposition 2.2(1) and the right commutes by Corollary 4.2. Since the last horizontal maps in both rows are natural isomorphisms by the homotopy invariance, we shall often identify the last two terms in both rows and use $j_{s, \leq t}^{*}$ and $\left(j_{s, \leq t} \circ g_{\leq t}\right)^{*}$ interchangeably.

Theorem 9.8. The maps $j_{s, \leq t}^{*}$ and $j_{s, t}^{*}$ are surjective and there are ring homomorphisms

$$
\begin{aligned}
& \overline{\mathrm{Sp}}_{X, s}^{\leq t}: \mathrm{CH}_{G}^{*}\left(X_{\leq t} ; \mathbb{Q}\right) \rightarrow \mathrm{CH}_{G}^{*}\left(N_{s, \leq t} ; \mathbb{Q}\right) \text { and } \\
& \overline{\mathrm{Sp}}_{X, s}^{t}: \mathrm{CH}_{G}^{*}\left(X_{t} ; \mathbb{Q}\right) \rightarrow \mathrm{CH}_{G}^{*}\left(N_{s, t} ; \mathbb{Q}\right)
\end{aligned}
$$

such that $i_{s, \leq t}^{*}=\overline{\mathrm{Sp}}_{X, s}^{\leq t} \circ j_{s, \leq t}^{*}$ and $i_{s, t}^{*}=\overline{\mathrm{Sp}}_{X, s}^{t} \circ j_{s, t}^{*}$. Moreover, both the squares in the following diagram commute:

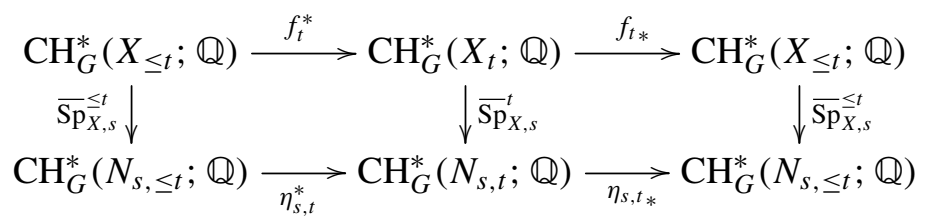


Proof. Using the results obtained so far in this section, one can define the specialization maps along the lines of the construction of such maps for $K$-theory in [Vezzosi and Vistoli 2003, Theorem 3.2; 2005]. However, it is not at all clear from the construction of the specialization maps in [Vezzosi and Vistoli 2005] that these maps have good functorial properties, and, in particular, if they are ring homomorphisms. Moreover, it is not clear if these maps will have the compatibility properties with the Chern character and Riemann-Roch maps (see [Krishna 2009b]) from the equivariant $K$-groups to higher Chow groups.

We give here a more direct and functorial construction of the specialization maps, which works both for the $K$-theory as well as the higher Chow groups, and the proof of various compatibilities of these maps then becomes essentially obvious. We give here the construction of these maps for the higher Chow groups. The same construction works also for the $K$-theory without any change.

First of all, using Corollary 9.7 and Proposition 9.2, we see that for $1 \leq s \leq n$ and $0 \leq t \leq s$, the map $\mathrm{CH}_{G}^{*}\left(X_{\leq s}\right) \rightarrow \mathrm{CH}_{G}^{*}\left(X_{\leq t}\right)$ is surjective. We now consider the commutative diagram

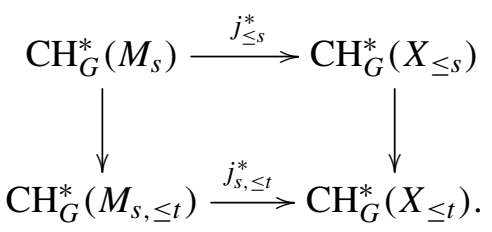

Since the composite map in the bottom row of (9-3) is identity, we see by the homotopy invariance that $j_{\leq s}^{*}$ is surjective. Thus $j_{s, \leq t}^{*}$ is also surjective. Applying this surjectivity for $j_{s, \leq t}^{*}$ and $j_{s, \leq t-1}^{*}$, we obtain the commutative diagram of Figure 1, which is such that the second and the third rows are exact. All the columns are exact by Corollary 9.7 and Proposition 9.2. We conclude that the localization sequence of the top row is also exact. This proves the surjectivity part of the theorem.

Next, we apply the self-intersection formula (Corollary 4.5) to the inclusions $i_{s, \leq t}$ and $i_{s, t}$ to see that the composites $i_{s, \leq t}^{*} \circ i_{s, \leq t_{*}}$ and $i_{s, t}^{*} \circ i_{s, t_{*}}$ are multiplication by the first Chern class $c_{1}^{G}$ of the corresponding normal bundles. But these normal bundles are the inverse images of a line bundle on $\mathbb{P}^{1}$. It follows that these normal bundles are trivial, because the restriction of any line bundle on $\mathbb{P}^{1}$ to $\infty \in \mathbb{P}^{1}$ and hence on the fiber over $\infty$ is clearly trivial. We conclude that the composites $i_{s, \leq t}^{*} \circ i_{s, \leq t_{*}}$ and $i_{s, t}^{*} \circ i_{s, t_{*}}$ are zero.

The diagram above now automatically defines the specializations $\overline{\mathrm{Sp}}_{X, s}^{\leq t}$ and $\overline{\mathrm{Sp}}_{X, s}^{t}$ and gives the desired factorization of $i_{s, \leq t}^{*}$ and $i_{s, t}^{*}$. Since $i_{s, t}^{*}$ and $j_{s, t}^{*}$ are ring homomorphisms, and since the latter is surjective as shown in 1 , we deduce that $\overline{\mathrm{Sp}}_{X, s}^{t}$ is also a ring homomorphism. The map $\overline{\mathrm{Sp}}_{X, s}^{\leq t}$ is a ring homomorphism for the same reason. 


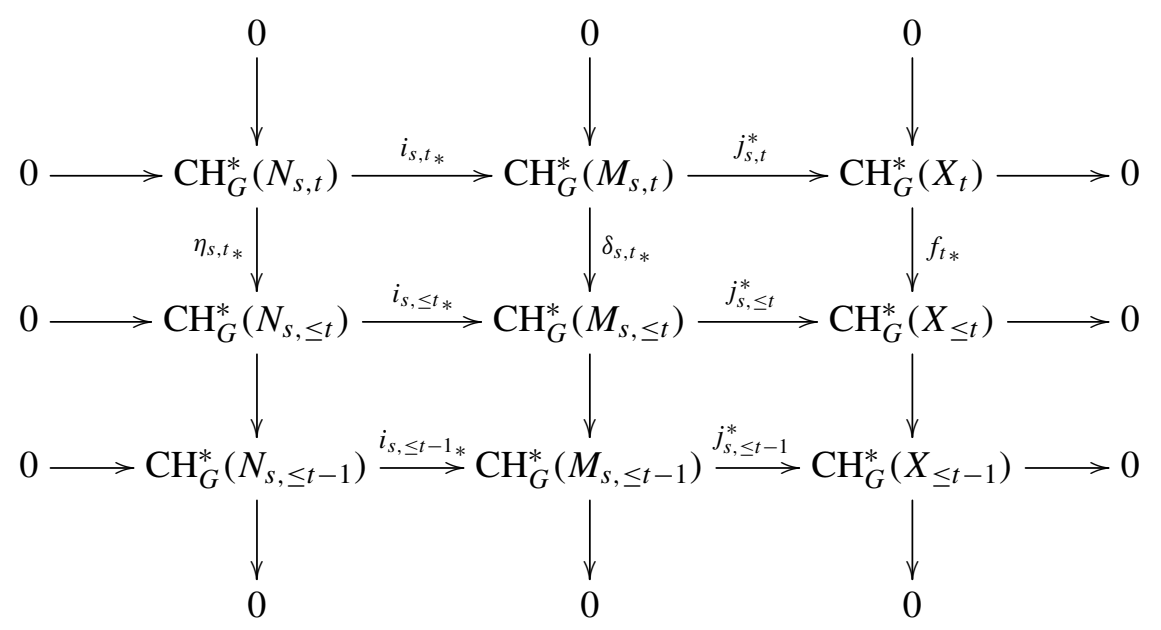

\section{Figure 1}

We are now left with the proof of the commutativity of (9-4). To prove that the right square commutes, we consider the following diagram.

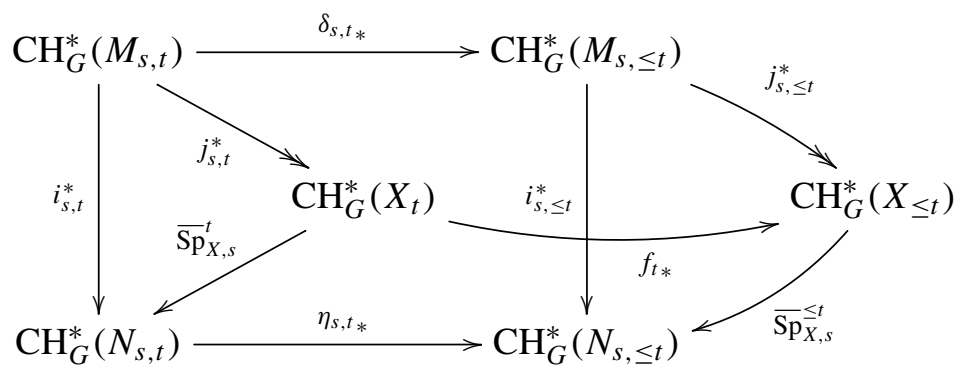

It is easy to check that $N_{s, \leq t}$ and $M_{s, t}$ are Tor-independent over $M_{s, \leq t}$ (see [Vezzosi and Vistoli 2005, Lemma 1]) and hence the back face of the diagram above commutes by Corollary 4.2. The upper face commutes by diagram 1 . Since $j_{s, t}^{*}$ is surjective, a diagram chase shows that the lower face also commutes, which is what we needed to prove.

Finally, since we have shown that $\eta_{s, t_{*}}$ is injective, and the right square commutes, it now suffices to show that the composite square in (9-4) commutes in order to show that the left square commutes.

By the projection formula, the composite maps $f_{t_{*}} \circ f_{t}^{*}$ and $\eta_{s, t_{*}} \circ \eta_{s, t}^{*}$ are multiplication by $f_{t_{*}}(1)$ and $\eta_{s, t_{*}}(1)$ respectively. Since

$$
\overline{\mathrm{Sp}}_{X, s}^{\leq t} \quad \text { and } \quad \overline{\mathrm{Sp}}_{X, s}^{t}
$$


are ring homomorphisms, it suffices to show that

$$
\overline{\mathrm{Sp}}_{X, s}^{\leq t}\left(f_{t *} \circ j_{s, t}^{*}(1)\right)=\overline{\operatorname{Sp}}_{X, s}^{\leq t}\left(f_{t *}(1)\right)=\eta_{s, t_{*}}(1) .
$$

But this follows directly from the commutativity of the right square.

\section{Decomposition theorem for equivariant higher Chow groups}

We use the specialization maps to prove the main decomposition theorem for the equivariant higher Chow groups of $X \in \mathscr{V}_{G}^{S}$, where $G$ is a split diagonalizable group. We continue with the notation of the previous section.

Proposition 10.1. The restriction maps

$$
\mathrm{CH}_{G}^{*}\left(X_{\leq s} ; \mathbb{Q}\right) \stackrel{\left(f_{s}^{*}, g_{s}^{*}\right)}{\longrightarrow} \mathrm{CH}_{G}^{*}\left(X_{s} ; \mathbb{Q}\right) \times \mathrm{CH}_{G}^{*}\left(X_{<s} ; \mathbb{Q}\right)
$$

define an isomorphism of rings

$$
\mathrm{CH}_{G}^{*}\left(X_{\leq s} ; \mathbb{Q}\right) \stackrel{\sim}{\longrightarrow} \mathrm{CH}_{G}^{*}\left(X_{s} ; \mathbb{Q}\right) \underset{\mathrm{CH}_{G}^{*}\left(N_{s}^{0} ; \mathbb{Q}\right)}{\times} \mathrm{CH}_{G}^{*}\left(X_{<s} ; \mathbb{Q}\right),
$$

where $\mathrm{CH}_{G}^{*}\left(X_{s} ; \mathbb{Q}\right) \stackrel{\eta_{s, \leq s-1}^{*}}{\longrightarrow} \mathrm{CH}_{G}^{*}\left(N_{s}^{0} ; \mathbb{Q}\right)$ is the pull-back

$$
\mathrm{CH}_{G}^{*}\left(X_{s} ; \mathbb{Q}\right) \stackrel{\sim}{\longrightarrow} \mathrm{CH}_{G}^{*}\left(N_{s} ; \mathbb{Q}\right) \rightarrow \mathrm{CH}_{G}^{*}\left(N_{s}^{0} ; \mathbb{Q}\right)
$$

and

$$
\mathrm{CH}_{G}^{*}\left(X_{<s} ; \mathbb{Q}\right) \stackrel{\overline{\mathrm{Sp}}_{X, s}^{\leq s-1}}{\longrightarrow} \mathrm{CH}_{G}^{*}\left(N_{s, \leq s-1} ; \mathbb{Q}\right)=\mathrm{CH}_{G}^{*}\left(N_{s}^{0} ; \mathbb{Q}\right)
$$

is the specialization map of Theorem 9.8.

Proof. We only need to identify the pull-back and the specialization maps with the appropriate maps of Proposition 9.2. In the diagram

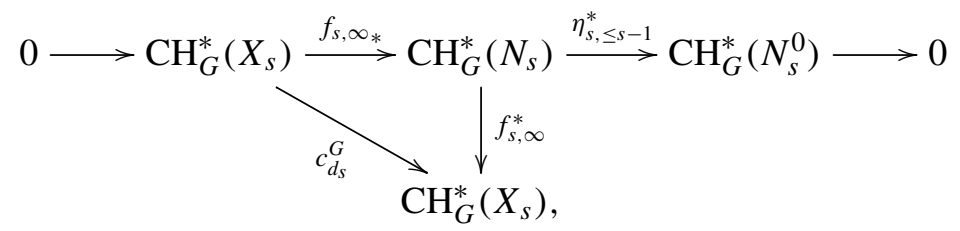

where $f_{s, \infty}: X_{s} \rightarrow N_{s}$ is the 0 -section embedding, the top sequence is exact, and the lower triangle commutes by Corollary 4.5. Since $f_{s, \infty}^{*}$ is an isomorphism, this immediately identifies the pull-back map of the proposition with the quotient map

$$
\mathrm{CH}_{G}^{*}\left(X_{s}\right) \rightarrow \frac{\mathrm{CH}_{G}^{*}\left(X_{s}\right)}{\left(c_{d_{s}}^{G}\left(N_{s}\right)\right)}
$$


Next, we consider the diagram

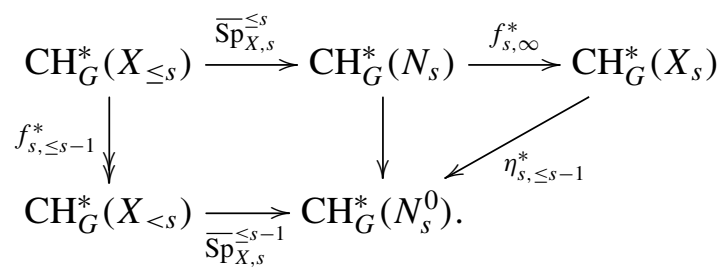

Since the left vertical arrow in the diagram above is surjective, we only need to show that

$$
\overline{\mathrm{Sp}}_{X, s}^{\leq s-1} \circ f_{s, \leq s-1}^{*}=\eta_{s, \leq s-1}^{*} \circ f_{s}^{*}
$$

in order to identify $\overline{\operatorname{Sp}}_{X, s}^{\leq s-1}$ with the map $j^{*}$ of Proposition 9.2. It is clear from the diagram 1 and the definition of the specialization maps that the left square in the diagram (10-1) commutes. We have just shown above that the right side triangle also commutes. This reduces us to showing that

$$
f_{s, \infty}^{*} \circ \overline{\mathrm{Sp}}_{X, s}^{\leq s}=f_{s}^{*} \text {. }
$$

If $X_{s} \times \mathbb{P}^{1} \stackrel{F_{s}}{\rightarrow} M_{s}$ denotes the embedding (see (4-1)), then for $x \in \mathrm{CH}_{G}^{*}\left(X_{\leq s}\right)$, we can write $x=j_{\leq s}^{*}(y)$ by Theorem 9.8. Then

$$
\begin{aligned}
f_{s, \infty}^{*} \circ \overline{\operatorname{Sp}}_{X, s}^{\leq s} \circ j_{\leq s}^{*}(y) & =f_{s, \infty}^{*} \circ i_{s, \leq s}^{*}(y)={ }^{\dagger} g_{\infty, \leq s}^{*} \circ F_{s}^{*}(y) \\
& =g_{0, \leq s}^{*} \circ F_{s}^{*}(y)=f_{s}^{*} \circ j_{\leq s}^{*}(y)=f_{s}^{*}(x),
\end{aligned}
$$

where ${ }^{\dagger}$ follows from Corollary 4.2. This proves (10-2) and the proposition.

We need the following algebraic result before we prove the main theorem. Let $A$ be a $\mathbb{Q}$-algebra (not necessarily commutative) and let $Z(A)$ denote the center of $A$. For any linear form $f(\underline{t})=\sum_{i=1}^{n} a_{i} t_{i}$ in $A\left[t_{1}, \ldots, t_{n}\right]$ such that $a_{i} \in \mathbb{Q}$ for each $i$, let $c(f)$ denote the vector $\left(a_{1}, \ldots, a_{n}\right) \in \mathbb{Q}^{n}$ consisting of the coefficients of the form $f$.

Lemma 10.2. Let $A$ be as above and let $S=\left\{f_{1}, \ldots, f_{s}\right\}$ be a set of linear forms in $A\left[t_{1}, \ldots, t_{n}\right]$ such that the vectors $\left\{c\left(f_{1}\right), \ldots, c\left(f_{s}\right)\right\}$ are pairwise nonproportional in $\mathbb{Q}^{n}$. Let

$$
\gamma_{j}=\sum_{i=0}^{d_{j}} m_{i}^{j} f_{j}^{i}
$$

such that $m_{d_{j}}^{j} \in \mathbb{Q}^{*}$ for $1 \leq j \leq s$, and $m_{j^{\prime}}^{j} \in Z(A)$ for all $j, j^{\prime}$. Then one has

$$
\left(\gamma_{1} \cdots \gamma_{s}\right)=\bigcap_{j=1}^{s}\left(\gamma_{j}\right)
$$

as ideals in $A\left[t_{1}, \ldots, t_{n}\right]$. 
Proof. Using a simple induction, it suffices to show that for $j \neq j^{\prime}$, the relation $\gamma_{j} \mid q \gamma_{j^{\prime}}$ implies that $\gamma_{j} \mid q$. So we can assume $S=\left\{f_{1}, f_{2}\right\}$. Since $c\left(f_{1}\right)$ and $c\left(f_{2}\right)$ are nonproportional, we can extend the set $\left\{c\left(f_{1}\right), c\left(f_{2}\right)\right\}$ to a basis $B$ of $\mathbb{Q}^{n}$. Applying the linear automorphism of $A\left[t_{1}, \ldots, t_{n}\right]$ given by the invertible matrix $B$, we can assume that $f_{j}=t_{j}$ for $j=1,2$. Now the proof follows along the same lines as the proof of [Vezzosi and Vistoli 2003, Lemma 4.9].

Theorem 10.3. Let $G$ be a split diagonalizable group of dimension $n$ and let $X \in \mathscr{V}_{G}^{S}$. The ring homomorphism

$$
\mathrm{CH}_{G}^{*}(X ; \mathbb{Q}) \rightarrow \prod_{s=0}^{n} \mathrm{CH}_{G}^{*}\left(X_{s} ; \mathbb{Q}\right)
$$

is injective. Moreover, its image consists of the $n$-tuples $\left(\alpha_{s}\right)$ in the product with the property that for each $s=1, \ldots, n$, the pull-back of $\alpha_{s} \in \mathrm{CH}_{G}^{*}\left(X_{s} ; \mathbb{Q}\right)$ in $\mathrm{CH}_{G}^{*}\left(N_{s, s-1} ; \mathbb{Q}\right)$ is the same as $\overline{\operatorname{Sp}}_{X, s}^{s-1}\left(\alpha_{s-1}\right) \in \mathrm{CH}_{G}^{*}\left(N_{s, s-1} ; \mathbb{Q}\right)$. In other words, there is a ring isomorphism

$$
\mathrm{CH}_{G}^{*}(X ; \mathbb{Q}) \stackrel{\sim}{\longrightarrow} \mathrm{CH}_{G}^{*}\left(X_{n} ; \mathbb{Q}\right) \underset{\mathrm{CH}_{G}^{*}\left(N_{n, n-1} ; \mathbb{Q}\right)}{\times} \cdots \underset{\mathrm{CH}_{G}^{*}\left(N_{1,0} ; \mathbb{Q}\right)}{\times} \mathrm{CH}_{G}^{*}\left(X_{0} ; \mathbb{Q}\right) .
$$

Proof. We prove by induction on the largest integer $s$ such that $X_{s} \neq \varnothing$.

If $s=0$, there is nothing to prove. If $s>0$, we have by induction

$$
\mathrm{CH}_{G}^{*}\left(X_{<s}\right) \stackrel{\cong}{\longrightarrow} \mathrm{CH}_{G}^{*}\left(X_{s-1}\right) \underset{\mathrm{CH}_{G}^{*}\left(N_{s-1, s-2}\right)}{\times} \cdots \underset{\mathrm{CH}_{G}^{*}\left(N_{1,0}\right)}{\times} \mathrm{CH}_{G}^{*}\left(X_{0}\right) .
$$

Using (10-3) and Proposition 10.1, it suffices to show that if $\alpha_{s} \in \mathrm{CH}_{G}^{*}\left(X_{s}\right)$ and if $\alpha_{<s} \in \mathrm{CH}_{G}^{*}\left(X_{<s}\right)$ with the restriction $\alpha_{s-1} \in \mathrm{CH}_{G}^{*}\left(X_{s-1}\right)$ are such that $\alpha_{s} \mapsto \alpha_{s}^{0} \in \mathrm{CH}_{G}^{*}\left(N_{s}^{0}\right)$ and $\alpha_{s} \mapsto \alpha_{s, s-1} \in \mathrm{CH}_{G}^{*}\left(N_{s, s-1}\right)$, then

$$
\overline{\mathrm{Sp}}_{X, s}^{\leq s-1}\left(\alpha_{<s}\right)=\alpha_{s}^{0} \quad \text { if and only if } \quad \overline{\operatorname{Sp}}_{X, s}^{s-1}\left(\alpha_{s-1}\right)=\alpha_{s, s-1} .
$$

Using the commutativity of the left square in Theorem 9.8, this is reduced to showing that the restriction map

$$
\mathrm{CH}_{G}^{*}\left(N_{s}^{0}\right) \rightarrow \mathrm{CH}_{G}^{*}\left(N_{s, s-1}\right)
$$

is injective.

To prove this, we first use Proposition 9.5 to assume that the toral component $T$ of the isotropy groups of the points of $X_{s}$ is fixed, and choose a splitting $G=D \times T$.

Now, following the proof of the analogous result for $K$-theory [Vezzosi and Vistoli 2003, Theorem 4.5], we can write

$$
N_{s}=E=\bigoplus_{i=1}^{q} E_{i} \quad \text { and } \quad N_{s, s-1}=\coprod_{i} E_{i}^{0},
$$


where each $E_{i}$ is of the form $\bigoplus E_{m_{j}} \otimes \chi_{i}^{m_{j}}$ such that for $i \neq i^{\prime}, \chi_{i}$ and $\chi_{i^{\prime}}$ are nonproportional characters of $T$, and $E_{i}^{0}$ is embedded in $E$ by setting all the other components equal to zero. Let $d_{i}=\operatorname{rank}\left(E_{i}\right)$.

Now we see from Proposition 10.1 that

$$
\begin{aligned}
& \operatorname{Ker}\left(\mathrm{CH}_{G}^{*}\left(X_{s}\right) \rightarrow \mathrm{CH}_{G}^{*}\left(N_{s, s-1}\right)\right)=\bigcap_{i}\left(c_{d_{i}}^{G}\left(E_{i}\right)\right), \\
& \operatorname{Ker}\left(\mathrm{CH}_{G}^{*}\left(X_{s}\right) \rightarrow \mathrm{CH}_{G}^{*}\left(N_{s}^{0}\right)\right)=\left(c_{d_{s}}^{G}\left(N_{s}\right)\right) \quad \text { with } d_{s}=\sum d_{i} .
\end{aligned}
$$

Put $\gamma_{i}=c_{d_{i}}^{G}\left(E_{i}\right)$ and $\gamma=c_{d_{s}}^{G}\left(N_{s}\right)$. Since the map $\mathrm{CH}_{G}^{*}\left(X_{s}\right) \rightarrow \mathrm{CH}_{G}^{*}\left(N_{s}^{0}\right)$ is surjective, showing the injectivity of the map in (10-4) is equivalent to showing that

$$
(\gamma)=\left(\prod_{i} \gamma_{i}\right)=\bigcap_{i}\left(\gamma_{i}\right)
$$

in $\mathrm{CH}_{D}^{*}\left(X_{s}\right)\left[t_{1}, \ldots, t_{s}\right]$.

However, we have seen in the proof of Proposition 9.4 that each $\gamma_{i}$ is of the form

$$
\gamma_{i}=u_{i}^{d_{i}}+\alpha_{d_{i}-1}^{i} u_{i}^{d_{i}-1}+\cdots+\alpha_{1}^{i} u_{i}+\alpha_{0}^{i},
$$

where $\alpha_{j}^{i} \in \mathrm{CH}_{D}^{*}\left(X_{s}, 0\right) \subseteq Z\left(\mathrm{CH}_{D}^{*}\left(X_{s}\right)\right)$ and $u_{i}=c_{1}^{T}\left(L_{\chi_{i}}\right)=\sum_{j=1}^{s} b_{j}^{i} t_{j} \neq 0$ in $\mathbb{Q}\left[t_{1}, \ldots, t_{s}\right]$. Moreover, the pairwise nonproportionality of $\chi_{i}$ implies the same for the vectors $\left\{c\left(u_{1}\right), \ldots, c\left(u_{q}\right)\right\}$ in $\mathbb{Q}^{s}$. We now apply Lemma 10.2 to conclude the proof of (10-5) and hence the theorem.

\section{Equivariant higher Chow groups of toric varieties}

In this section, we apply our decomposition theorem to give explicit descriptions of the equivariant higher Chow groups of smooth toric varieties. An analogous description of the equivariant cohomology of such varieties was earlier given by Bifet, De Concini and Procesi in [Bifet et al. 1990] and such a description of the equivariant $K$-theory was given by Vezzosi and Vistoli [2003]. Brion [1997, Theorem 5.4] had proven similar results for the classical equivariant Chow groups of toric varieties. As a consequence of our descriptions of the equivariant higher Chow groups, we shall obtain formulas for the ordinary higher Chow groups (or motivic cohomology) of smooth toric varieties.

Let $T$ be a split torus of rank $n$ over $k$. Let $M=\operatorname{Hom}\left(\mathbb{G}_{m}, T\right)$ be the lattice of the one-parameter subgroups of $T$ and let $M^{\vee}$ be the character lattice of $T$. Let $\Delta$ be a fan in $M_{\mathbb{R}}$ and let $\Delta_{1}$ and $\Delta_{\max }$ denote the subsets of the one-dimensional cones and the maximal cones in $\Delta$, respectively.

Let $X=X(\Delta)$ be the smooth toric variety associated to the fan $\Delta$. The smoothness of $X$ is equivalent to the condition that every positive dimensional cone of $\Delta$ is generated by it edges such that the primitive vectors along these edges form a 
subset of a basis of $M$. In this case, there is an one-to-one correspondence between the $T$-orbits in $X$ and the cones in $\Delta$. For every cone $\sigma \in \Delta$, the corresponding orbit $O_{\sigma}$ is isomorphic to the torus $T / T_{\sigma}$, where $T_{\sigma}$ is associated to the sublattice $M_{\sigma}$ of $M$ generated by $\sigma \cap M$. Under this isomorphism, the origin (identity point) of $T / T_{\sigma}$ corresponds to the distinguished $k$-rational point $x_{\sigma}$ of $O_{\sigma}$. In particular, for every $0 \leq s \leq n, X_{s}$ is of the form

$$
X_{s}=\coprod_{\operatorname{dim}(\sigma)=s} O_{\sigma} \cong \coprod_{\operatorname{dim}(\sigma)=s} T / T_{\sigma} .
$$

We shall write $\tau \leq \sigma$ if $\tau$ is a face of $\sigma$ as cones in $\Delta$. The orbit closure $V_{\sigma}$ of $O_{\sigma}$ is the toric variety associated to the fan $*(\sigma)=\{\tau \in \Delta \mid \sigma \leq \tau\}$, called the star of $\sigma$. Moreover, it is clear from the characterization of the smoothness of toric varieties that $V_{\sigma}$ is also smooth and is the disjoint union of all orbits $O_{\tau}$ such that $\sigma$ is a face of $\tau$. In particular, $O_{\sigma}$ is closed in $X$ if and only if $\sigma \in \Delta_{\max }$. The following is our first description of the equivariant higher Chow groups of smooth toric varieties.

Theorem 11.1. Let $X=X(\Delta)$ be a smooth toric variety associated to a fan $\Delta$ in $M_{\mathbb{B}}$. There is an injective homomorphism of $S$-algebras

$$
\Phi_{X}: \mathrm{CH}_{T}^{*}(X ; \mathbb{Q}) \rightarrow \prod_{\sigma \in \Delta_{\max }} \mathrm{CH}^{*}(k ; \mathbb{Q}) \otimes S\left(T_{\sigma} ; \mathbb{Q}\right) .
$$

An element

$$
\left(a_{\sigma}\right) \in \prod_{\sigma \in \Delta_{\max }} \mathrm{CH}^{*}(k ; \mathbb{Q}) \otimes S\left(T_{\sigma} ; \mathbb{Q}\right)
$$

is in the image of this homomorphism if and only if for any two maximal cones $\sigma_{1}$ and $\sigma_{2}$, the restrictions of $a_{\sigma_{1}}$ and $a_{\sigma_{2}}$ to $\mathrm{CH}^{*}(k ; \mathbb{Q}) \otimes S\left(T_{\sigma_{1} \cap \sigma_{2}} ; \mathbb{Q}\right)$ coincide.

Proof. We only need to appropriately identify the various terms and the maps in the statement of Theorem 10.3. We follow the notation of Section 9 and Section 10. It follows from [Vezzosi and Vistoli 2003, Lemma 6.1] that for every $s \geq 1$, there is a canonical isomorphism

$$
N_{s, s-1}=\coprod_{\substack{\sigma \in \Delta \\ \operatorname{dim}(\sigma)=s}} \coprod_{\tau \in \partial \sigma} O_{\tau}
$$

Furthermore, for each $s$-dimensional cone $\sigma$ and $\tau \in \partial \sigma$, the composition of the map

$$
\mathrm{Sp}_{X, s}^{s-1}: \mathrm{CH}_{T}^{*}\left(X_{s-1}\right)=\prod_{\substack{\tau \in \Delta \\ \operatorname{dim}(\tau)=s-1}} \mathrm{CH}_{T}^{*}\left(O_{\tau}\right) \rightarrow \mathrm{CH}_{T}^{*}\left(N_{s, s-1}\right)=\prod_{\substack{\sigma \in \Delta \\ \operatorname{dim}(\sigma)=s}} \prod_{\tau \in \partial \sigma} \mathrm{CH}_{T}^{*}\left(O_{\tau}\right)
$$


with the projection

$$
\operatorname{Pr}_{\sigma, \tau}: \prod_{\substack{\sigma \in \Delta \\ \operatorname{dim}(\sigma)=s}} \prod_{\tau \in \partial \sigma} \mathrm{CH}_{T}^{*}\left(O_{\tau}\right) \rightarrow \mathrm{CH}_{T}^{*}\left(O_{\tau}\right)
$$

is the projection map

$$
\prod_{\substack{\tau \in \Delta \\ \mathrm{m}(\tau)=s-1}} \mathrm{CH}_{T}^{*}\left(O_{\tau}\right) \rightarrow \mathrm{CH}_{T}^{*}\left(O_{\tau}\right) .
$$

The identification above of the specialization maps in [Vezzosi and Vistoli 2003, Lemma 6.1] was shown for the equivariant $K$-theory, but the same holds in the present case as well without any modification in view of the construction of these specializations for higher Chow groups in Section 9.

It also follows from Corollary 3.2 and Theorem 3.5 that

$$
\mathrm{CH}_{T}^{*}\left(O_{\sigma}\right) \cong \mathrm{CH}_{T}^{*}\left(T / T_{\sigma}\right) \cong \mathrm{CH}_{T_{\sigma}}^{*}(k) \cong \mathrm{CH}^{*}(k) \otimes S\left(T_{\sigma}\right) .
$$

We conclude now from (11-1) and Theorem 10.3 that $\mathrm{CH}_{T}^{*}(X)$ is a subring of $\prod_{\sigma \in \Delta} \mathrm{CH}^{*}(k) \otimes S\left(T_{\sigma}\right)$, consisting of elements $\left(a_{\sigma}\right)$ with the property that the restriction of $a_{\sigma} \in \mathrm{CH}^{*}(k) \otimes S\left(T_{\sigma}\right)$ to $\mathrm{CH}^{*}(k) \otimes S\left(T_{\tau}\right)$ coincides with $a_{\tau}$ whenever $\tau \leq \sigma$. The theorem now follows from the fact that every cone in $\Delta$ is contained in a maximal cone in $\Delta$.

As an immediate consequence of Theorem 11.1, we obtain the following localization theorem for the equivariant higher Chow groups of smooth projective toric varieties. This was earlier proven for $\mathrm{CH}_{T}^{*}(X, 0)$ by Brion [1997, Theorem 3.4].

Corollary 11.2. Let $X=X(\Delta)$ be a smooth projective toric variety and $\left\{x_{1}, \ldots, x_{r}\right\}$ be the fixed point locus of $X$. Then the map

$$
\mathrm{CH}_{T}^{*}(X ; \mathbb{Q}) \rightarrow \mathrm{CH}_{T}^{*}\left(X^{T} ; \mathbb{Q}\right) \stackrel{\sim}{\longrightarrow}\left(\mathrm{CH}^{*}(k ; \mathbb{Q})\left[t_{1}, \ldots, t_{n}\right]\right)^{r}
$$

is injective and its image is the set of all $n$-tuples $\left(f_{1}, \ldots, f_{n}\right)$ such that $f_{i} \equiv f_{j}$ $(\bmod \chi)$ whenever $x_{i}$ and $x_{j}$ lie on a $T$-invariant smooth irreducible curve on which $T$ acts through its character $\chi$.

Proof. This follows directly from Theorem 11.1 once we observe that the fixed points of $X$ for the torus action are same as the $T$-orbits corresponding to the maximal cones in $\Delta$ that are $n$-dimensional. Moreover, the orbit closures corresponding to the codimension one cones in $\Delta$ are the smooth $T$-invariant curves.

11a. Stanley-Reisner presentation. Using Theorem 11.1, we now give another explicit presentation of the equivariant higher Chow groups of smooth toric varieties. This presentation is analogous to the Stanley-Reisner presentation of the equivariant cohomology in [Bifet et al. 1990, Theorem 8] and equivariant $K$-theory in [Vezzosi 
and Vistoli 2003, Theorem 6.1]. This presentation has the advantage that it can often be used to describe the ordinary higher Chow groups of smooth toric varieties.

Let $T$ be a split torus of rank $n$ and let $M$ denote the lattice of the one-parameter subgroups of $T$. Let $X=X(\Delta)$ be a smooth toric variety associated to a fan $\Delta$ in $M_{\mathbb{R}}$. For $r \geq 1$, let $\Delta_{r}$ denote the set of $r$-dimensional cones in $\Delta$. For $\sigma \in \Delta_{\max }$, let $M_{\sigma}$ denote the sublattice of one-parameter subgroups of $T_{\sigma}$ so that $\widehat{T}_{\sigma}=M_{\sigma}^{\vee}$ as an abelian group. For any $\rho \in \Delta_{1}$, let $v_{\rho}$ denote the generator of the monoid $\rho \cap M$. Note that if $\left\{\rho_{1}, \ldots, \rho_{s}\right\}$ is the set of one-dimensional faces of $\sigma \in \Delta_{\max }$, then the smoothness of $X$ implies that $\left\{v_{\rho_{1}}, \ldots, v_{\rho_{s}}\right\}$ is a basis of $M_{\sigma}$. Let $\left\{v_{\rho_{1}}^{\vee}, \ldots, v_{\rho_{s}}^{\vee}\right\}$ denote the dual basis of $M_{\sigma}^{\vee}$.

We recall that for $\sigma \in \Delta$, there is a canonical isomorphism of abelian groups $\widehat{T}_{\sigma} \hookrightarrow S\left(T_{\sigma}\right)_{1}$ given by $\chi \mapsto c_{1}^{T_{\sigma}}\left(L_{\chi}\right)$. For each $\rho \in \Delta_{1}$, we define an element $u_{\rho}=\left(u_{\rho}^{\sigma}\right) \in \prod_{\sigma \in \Delta_{\max }} S\left(T_{\sigma}\right)$ such that

$$
u_{\rho}^{\sigma}= \begin{cases}v_{\rho}^{\vee} & \text { if } \rho \leq \sigma \\ 0 & \text { otherwise }\end{cases}
$$

Then $u_{\rho}$ has the property that for all $\sigma_{1}, \sigma_{2} \in \Delta_{\max }$, the restrictions of $u_{\rho}^{\sigma_{1}} \in \widehat{T}_{\sigma_{1}}$ and $u_{\rho}^{\sigma_{2}} \in \widehat{T}_{\sigma_{2}}$ in $\widehat{T}_{\sigma_{1} \cap \sigma_{2}}$ coincide.

We have the obvious inclusion

$$
\prod_{\sigma \in \Delta_{\max }} S\left(T_{\sigma}\right) \subseteq \prod_{\sigma \in \Delta_{\max }} \mathrm{CH}^{*}(k) \otimes S\left(T_{\sigma}\right)
$$

and using the description of $\mathrm{CH}_{T}^{*}(X)$ in Theorem 11.1 and the description of $u_{\rho}$ above, we can consider these $u_{\rho}$ as elements of the ring $\mathrm{CH}_{T}^{*}(X)$. In other words, we get a bigraded $\mathrm{CH}^{*}(k)$-algebra homomorphism

$$
\mathrm{CH}^{*}(k)\left[t_{\rho}\right] \rightarrow \mathrm{CH}_{T}^{*}(X), \quad t_{\rho} \mapsto u_{\rho},
$$

where $\mathrm{CH}^{*}(k)\left[t_{\rho}\right]$ is the polynomial algebra $\mathrm{CH}^{*}(k)\left[t_{\rho} \mid \rho \in \Delta_{1}\right]$.

If $S$ is a subset of $\Delta_{1}$ that is not contained in any maximal cone of $\Delta$, then for any given $\sigma \in \Delta_{\max }$, there is one $\rho \in S$ such that $\rho \not \leq \sigma$. This implies in particular that $u_{\rho}^{\sigma}=0$. We conclude from this that the elements $u_{\rho}$ satisfy the relation

$$
\prod_{\rho \in S} u_{\rho}=0 \quad \text { in } \mathrm{CH}_{T}^{*}(X)
$$

whenever $S \subseteq \Delta_{1}$ is such that it is not contained in any maximal cone of $\Delta$. We shall denote the collection of all such subsets of $\Delta_{1}$ by $\Delta_{1}^{0}$. We conclude that if $I_{\Delta}$ denotes the graded ideal of $\mathrm{CH}^{*}(k)\left[t_{\rho}\right]$ generated by the set of monomials 
$\left\{\prod_{\rho \in S} t_{\rho} \mid S \in \Delta_{1}^{0}\right\}$, then there is a $\mathrm{CH}^{*}(k)$-algebra homomorphism

$$
\Psi_{X}: \frac{\mathrm{CH}^{*}(k)\left[t_{\rho}\right]}{I_{\Delta}} \rightarrow \mathrm{CH}_{T}^{*}(X), \quad t_{\rho} \mapsto u_{\rho} .
$$

Note also that any character $\chi \in M^{\vee}$ defines multiplication by the element $t_{\chi}=\sum_{\rho \in \Delta_{1}}\left\langle\chi, v_{\rho}\right\rangle t_{\rho}$ in $\mathrm{CH}^{*}(k)\left[t_{\rho}\right]$, and this makes the term on the left hand side of (11-7) an $S$-algebra and $\Psi_{X}$ is also an $S$-algebra homomorphism. Furthermore, it is easy to check from the definition of $u_{\rho}$ that it is the fundamental class (see [Edidin and Graham 1998, Section 2]) of the $T$-equivariant Chow cycle

$$
\left[V_{\rho} \rightarrow X\right] \in \mathrm{CH}_{T}^{1}(X, 0) \subsetneq \mathrm{CH}_{T}^{*}(X),
$$

where $V_{\sigma}$ is the orbit closure in $X$ associated to a cone $\sigma \in \Delta$.

Theorem 11.3. For a smooth toric variety $X=X(\Delta)$ associated to a fan $\Delta$ in $M_{\mathbb{R}}$, the homomorphism $\Psi_{X}$ is an isomorphism with rational coefficients.

Proof. We prove the theorem by induction on the number of maximal cones in $\Delta$. Suppose $\Delta_{\max }=\{\sigma\}$ is a singleton set. In that case, $\sigma$ is the only maximal cone and $X=U_{\sigma}$ is a $T$-equivariant vector bundle over $O_{\sigma}$ such that the inclusion

$$
O_{\sigma} \stackrel{i_{\sigma}}{\longrightarrow} X
$$

is the zero-section embedding. Hence, we conclude from (11-3) that there are isomorphisms

$$
\mathrm{CH}_{T}^{*}(X) \underset{i_{\sigma}^{*}}{\stackrel{\sim}{\longrightarrow}} \mathrm{CH}_{T}^{*}\left(O_{\sigma}\right) \cong \mathrm{CH}^{*}(k) \otimes S\left(T_{\sigma}\right)=\mathrm{CH}^{*}(k)\left[t_{1}, \ldots, t_{s}\right],
$$

where $s$ is the dimension of $\sigma$. It is also clear in this case that the ideal $I_{\Delta}$ in (11-7) is zero. Hence, we have isomorphisms

$$
\mathrm{CH}^{*}(k)\left[t_{1}, \ldots, t_{s}\right] \underset{\Psi_{X}}{\stackrel{\sim}{\longrightarrow}} \mathrm{CH}_{T}^{*}(X) \underset{\Phi_{X}}{\stackrel{\sim}{\longrightarrow}} \mathrm{CH}^{*}(k) \otimes S\left(T_{\sigma}\right) .
$$

We consider now the general case. We assume that $\left|\Delta_{\max }\right| \geq 2$ and choose a maximal cone $\sigma$ of dimension $s \geq 1$ in $\Delta$. Let $X^{\prime}=X^{\prime}\left(\Delta^{\prime}\right)$ be the toric variety associated to the fan $\Delta^{\prime}=\Delta \backslash\{\sigma\}$. Note that $O_{\sigma}$ is a closed $T$-orbit in $X$ and $X^{\prime}$ is the complement of $O_{\sigma}$ in $X$. Let $U_{\sigma} \subset X$ be the principal open set associated to the fan consisting of all faces of $\sigma$ and let $U^{\prime}$ be the complement of $O_{\sigma}$ in $U_{\sigma}$. Then $U^{\prime}$ is nothing but the complement of the zero-section in the $T$-equivariant vector bundle $U_{\sigma} \rightarrow O_{\sigma}$. Let $i_{\sigma}: O_{\sigma} \hookrightarrow X$ and $j_{\sigma}: X^{\prime} \hookrightarrow X$ denote the $T$-invariant closed and open embeddings respectively. Let $S_{\sigma}=\left\{\rho_{1}, \ldots, \rho_{s}\right\}$ be the set of one-dimensional faces of $\sigma$ and set

$$
x_{\sigma}=\prod_{j=1}^{s} t_{\rho_{j}} \in \frac{\mathrm{CH}^{*}(k)\left[t_{\rho}\right]}{I_{\Delta}} \quad \text { and } \quad y_{\sigma}=\prod_{j=1}^{s} u_{\rho_{j}} \in \mathrm{CH}_{T}^{*}(X) .
$$


Since $N_{O_{\sigma} / X}=N_{O_{\sigma} / U_{\sigma}}$ and since the latter is of the form $\bigoplus_{j=1}^{s} L_{\chi_{j}}$, where $\left\{\chi_{1}, \ldots, \chi_{s}\right\}$ is a basis of $\widehat{T}_{\sigma}$, it follows from the definition of the elements $u_{\rho}$ (see Proposition 9.4) that

$$
c_{s}^{T}\left(N_{O_{\sigma} / X}\right)=y_{\sigma} \in \mathrm{CH}_{T}^{*}(X) .
$$

We consider the diagram

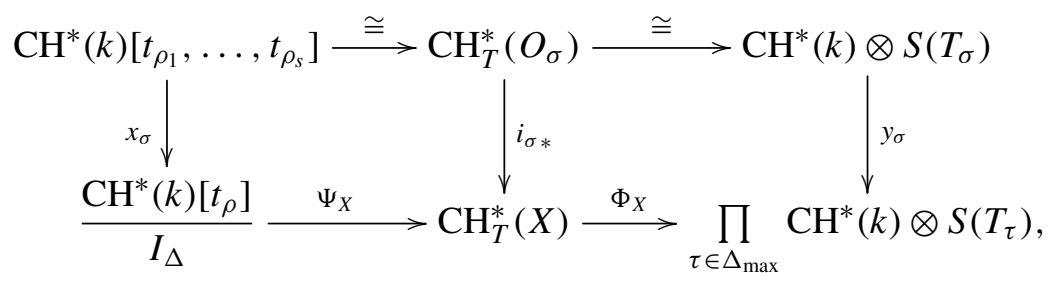

where the horizontal maps on the top are the obvious isomorphisms taking $t_{\rho_{j}}$ to $u_{\rho_{j}}$. The left and the right vertical maps are the multiplication by the indicated elements in the target rings. We claim that all the vertical arrows are injective and the left square in this diagram commutes.

To prove the claim, notice that the composite outer square clearly commutes by the definition of $x_{\sigma}$ and $y_{\sigma}$ and the map $\Psi_{X}$. Since $\Phi_{X}$ is injective by Theorem 11.1, we only need to show that the right square commutes and the right vertical arrow is injective to prove the claim.

We first observe that the right vertical arrow is the multiplication by $y_{\sigma}$ on the factor $\mathrm{CH}^{*}(k) \otimes S\left(T_{\sigma}\right)$ and is zero on the other factors of $\prod_{\tau \in \Delta_{\max }} \mathrm{CH}^{*}(k) \otimes S\left(T_{\tau}\right)$. Thus the required injectivity is equivalent to showing that the multiplication by $y_{\sigma}$ is injective in $\mathrm{CH}^{*}(k) \otimes S\left(T_{\sigma}\right)$. We can thus assume that $X=U_{\sigma}$ and then $\mathrm{CH}_{T}^{*}(X) \cong \mathrm{CH}^{*}(k)\left[t_{1}, \ldots, t_{s}\right]$. In this case, $y$ is just the element $t_{1} \cdots t_{s}$ and hence is a nonzero divisor in $\mathrm{CH}^{*}(k)\left[t_{1}, \ldots, t_{s}\right]$.

To show the commutativity of the right square, we observe from the proof of Theorem 11.1 that $\Phi_{X}$ is simply the product of the pull-back maps

$$
i_{\tau}^{*}: \mathrm{CH}_{T}^{*}(X) \rightarrow \mathrm{CH}_{T}^{*}\left(O_{\tau}\right) \text { for } \tau \in \Delta_{\max } \text {. }
$$

Hence the composite $\Phi_{X} \circ i_{\sigma *}$ is $i_{\sigma}^{*} \circ i_{\sigma *}$ on the factor $\mathrm{CH}_{T}^{*}\left(O_{\sigma}\right)$ and zero on the other factors of $\prod_{\tau \in \Delta_{\max }} \mathrm{CH}_{T}^{*}\left(O_{\tau}\right)$. Since we have just seen that the composite

$$
\mathrm{CH}_{T}^{*}\left(O_{\sigma}\right) \stackrel{y_{\sigma}}{\rightarrow} \prod_{\tau \in \Delta_{\max }} \mathrm{CH}_{T}^{*}\left(O_{\tau}\right)
$$


is of similar type, we are reduced to showing that the triangle

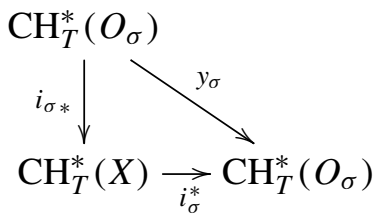

commutes. But this follows immediately from Corollary 4.5 and (11-8). This proves the claim.

To complete the proof of the theorem, we now consider the diagram

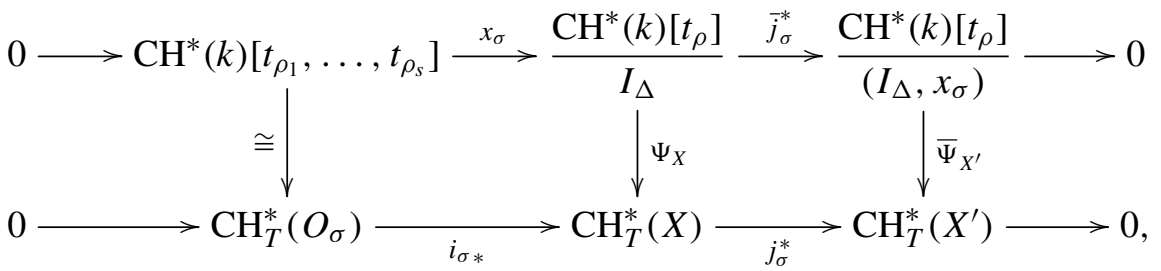

where $\bar{j}_{\sigma}^{*}$ is the natural quotient map by the ideal $\left(x_{\sigma}\right)$ in $\mathrm{CH}^{*}(k)\left[t_{\rho}\right] / I_{\Delta}$. Note that the image of the first map in the top row is the ideal $\left(x_{\sigma}\right)$ because the product of $x_{\sigma}$ with any $t_{\rho}$ for $\rho \notin\left\{\rho_{1}, \ldots, \rho_{s}\right\}$ is zero.

The left square in this diagram commutes and the first maps in both the rows are injective by the claim above. The bottom row is exact by Proposition 2.2. Since $\sigma$ is not a cone of $\Delta^{\prime}$, the element $x_{\sigma}$ is zero in $\mathrm{CH}^{*}(k)\left[t_{\rho}, \rho \in \Delta^{\prime}{ }_{1}\right] / I_{\Delta^{\prime}}$ and hence the map $j_{\sigma}^{*} \circ \Psi_{X}$ has a factorization:

$$
\frac{\mathrm{CH}^{*}(k)\left[t_{\rho}\right]}{I_{\Delta}} \rightarrow \frac{\mathrm{CH}^{*}(k)\left[t_{\rho}\right]}{\left(I_{\Delta}, x_{\sigma}\right)} \rightarrow \frac{\mathrm{CH}^{*}(k)\left[t_{\rho}, \rho \in \Delta^{\prime}{ }_{1}\right]}{I_{\Delta^{\prime}}} \stackrel{\Psi_{X^{\prime}}}{\longrightarrow} \mathrm{CH}_{T}^{*}\left(X^{\prime}\right),
$$

where the middle arrow is the natural map of the Stanley-Reisner rings induced by the inclusion of the fans $\Delta^{\prime} \subset \Delta$. Letting $\bar{\Psi}_{X^{\prime}}$ denote the composite

$$
\frac{\mathrm{CH}^{*}(k)\left[t_{\rho}\right]}{\left(I_{\Delta}, x_{\sigma}\right)} \rightarrow \frac{\mathrm{CH}^{*}(k)\left[t_{\rho}, \rho \in \Delta_{1}^{\prime}\right]}{I_{\Delta^{\prime}}} \stackrel{\Psi_{X^{\prime}}}{\longrightarrow} \mathrm{CH}_{T}^{*}\left(X^{\prime}\right),
$$

we see that the right square in the diagram (11-10) also commutes.

If all the cones of $\Delta$ are at most one-dimensional, then $x_{\sigma}=t_{\rho}$, where $\rho=\sigma$ and it is obvious that $\mathrm{CH}^{*}(k)\left[t_{\rho}\right] /\left(I_{\Delta}, x_{\sigma}\right)$ is the Stanley-Reisner ring associated to the fan $\Delta^{\prime}$. If $\Delta$ has a cone of dimension at least two, we can assume that $\sigma$ is of dimension at least two. In that case, we have $\Delta^{\prime}{ }_{1}=\Delta_{1}$ and the natural inclusion $\Delta_{1}^{0} \subseteq \Delta^{\prime 0}{ }_{1}^{0}$ gives the equality $\Delta^{\prime 0}{ }_{1}^{0}=\Delta_{1}^{0} \amalg\left\{S_{\sigma}\right\}$. In particular, we have

$$
\frac{\mathrm{CH}^{*}(k)\left[t_{\rho}, \rho \in \Delta_{1}\right]}{\left(I_{\Delta}, x_{\sigma}\right)} \underset{\sim}{\sim} \frac{\mathrm{CH}^{*}(k)\left[t_{\rho}, \rho \in \Delta^{\prime}{ }_{1}\right]}{I_{\Delta^{\prime}}} .
$$


On the other hand, $\Delta^{\prime}$ is a fan with smaller number of maximal cones than in $\Delta$ and $X^{\prime}=X^{\prime}\left(\Delta^{\prime}\right)$. Hence the map

$$
\frac{\mathrm{CH}^{*}(k)\left[t_{\rho}, \rho \in \Delta^{\prime}{ }_{1}\right]}{I_{\Delta^{\prime}}} \stackrel{\Psi_{X^{\prime}}}{\longrightarrow} \mathrm{CH}_{T}^{*}\left(X^{\prime}\right)
$$

is an isomorphism by induction. We conclude that the map $\bar{\Psi}_{X^{\prime}}$ in the diagram (11-10) is an isomorphism. A diagram chase in (11-10) now shows that $\Psi_{X}$ is also an isomorphism.

As an important application of Theorem 11.3, we obtain the following presentation of the ordinary higher Chow groups (motivic cohomology groups) of smooth toric varieties. An explicit description of $\mathrm{CH}^{*}(X, 0)$ for a smooth projective toric variety $X$ was given in [Fulton 1993, Proposition 5.2]. The following result extends this to all smooth toric varieties, not necessarily projective. In fact, such a description extends to all higher Chow groups of smooth projective toric varieties. In particular, we obtain another proof of Corollary 7.3 with rational coefficients. Recall that for every $\sigma \in \Delta$, the orbit closure $V_{\sigma}=\overline{O_{\sigma}}$ in $X$ is a $T$-invariant Weil divisor and defines a unique element $\left[V_{\sigma}\right] \in \mathrm{CH}^{1}(X, 0)$.

Corollary 11.4. Let $X=X(\Delta)$ be a smooth projective toric variety. Then the assignment $t_{\rho} \mapsto\left[V_{\sigma}\right]$ defines a $\mathrm{CH}^{*}(k ; \mathbb{Q})$-algebra isomorphism

$$
\bar{\Psi}_{X}: \frac{\mathrm{CH}^{*}(k ; \mathbb{Q})\left[t_{\rho}\right]}{\left(I_{\Delta}, \sum_{\rho \in \Delta_{1}}\left\langle\chi, v_{\rho}\right\rangle t_{\rho}\right)} \rightarrow \mathrm{CH}^{*}(X ; \mathbb{Q}),
$$

where $\chi$ runs over $M^{\vee}$.

If $X$ is not necessarily projective, the map

$$
\frac{\mathbb{Q}\left[t_{\rho}\right]}{\left(I_{\Delta}, \sum_{\rho \in \Delta_{1}}\left\langle\chi, v_{\rho}\right\rangle t_{\rho}\right)} \rightarrow \mathrm{CH}^{*}(X, 0 ; \mathbb{Q})
$$

is a ring isomorphism.

Proof. We have already seen before that every character $\chi \in M^{\vee}$ acts on $\mathrm{CH}^{*}(k)\left[t_{\rho}\right]$ by multiplication with the element $\sum_{\rho \in \Delta_{1}}\left\langle\chi, v_{\rho}\right\rangle t_{\rho}$ which makes the left hand side of (11-11) an $S$-algebra. The corollary now follows directly from Theorem 11.3 and [Krishna 2009a, Theorem 1.3]. The second isomorphism follows in the same way from Theorem 11.3 and [Brion 1997, Corollary 2.3].

Corollary 11.5. Let $X=X(\Delta)$ be a smooth toric variety. Then there are canonical ring isomorphisms

$$
\begin{aligned}
& \mathrm{CH}^{*}(k ; \mathbb{Q}) \otimes \mathrm{CH}_{T}^{*}(X, 0 ; \mathbb{Q}) \stackrel{\sim}{\rightarrow} \mathrm{CH}_{T}^{*}(X ; \mathbb{Q}), \\
& \mathrm{CH}^{*}(k ; \mathbb{Q}) \otimes \mathrm{CH}^{*}(X, 0 ; \mathbb{Q}) \stackrel{\sim}{\longrightarrow} \mathrm{CH}^{*}(X ; \mathbb{Q}) .
\end{aligned}
$$


Proof. It follows from (11-7) that $\mathrm{CH}^{*}(k) \otimes\left(\mathbb{Q}\left[t_{\rho}\right] / I_{\Delta}\right) \stackrel{\sim}{\rightarrow} \mathrm{CH}^{*}(k)\left[t_{\rho}\right] / I_{\Delta}$. The first part of the corollary now follows directly from Theorem 11.3. The second part follows from the first and [Krishna 2009a, Theorem 1.1], which says that there is a convergent spectral sequence

$$
\operatorname{Tor}_{p}^{S}\left(\mathbb{Q}, \mathrm{CH}_{T}^{*}(X, q)\right) \Rightarrow \mathrm{CH}^{*}(X, p+q) .
$$

Remark 11.6. All the results in this section about the (equivariant) higher Chow groups of smooth toric varieties have been stated over the rationals. However, an attentive reader can check that these results (and the proofs) for the subrings $\mathrm{CH}_{T}^{*}(X, 0)$ and $\mathrm{CH}^{*}(X, 0)$ hold true with the integral coefficients. The basic reason is that $\mathrm{CH}_{T}^{*}(k, 0)$ and $\mathrm{CH}^{*}(k, 0)$ are torsion-free abelian groups. But this is false for the higher Chow groups of $k$.

\section{Acknowledgments}

I wish to thank Angelo Vistoli, who drew my attention to the reconstruction theorem for equivariant $K$-theory in [Vezzosi and Vistoli 2003] and motivated me to think about such questions for equivariant higher Chow groups. I would also like to thank the referee for carefully reading this paper and for giving valuable input to improve its presentation.

\section{References}

[Bifet et al. 1990] E. Bifet, C. De Concini, and C. Procesi, "Cohomology of regular embeddings", Adv. Math. 82:1 (1990), 1-34. MR 91h:14052 Zbl 0743.14018

[Bloch 1986] S. Bloch, "Algebraic cycles and higher K-theory", Adv. in Math. 61:3 (1986), 267-304. MR 88f:18010 Zbl 0608.14004

[Borel 1991] A. Borel, Linear algebraic groups, 2nd ed., Graduate Texts in Mathematics 126, Springer, New York, 1991. MR 92d:20001 Zbl 0726.20030

[Brion 1997] M. Brion, "Equivariant Chow groups for torus actions", Transform. Groups 2:3 (1997), 225-267. MR 99c:14005 Zbl 0916.14003

[Demazure 1973] M. Demazure, "Invariants symétriques entiers des groupes de Weyl et torsion”, Invent. Math. 21 (1973), 287-301. MR 49\#7268 Zbl 0269.22010

[Demazure 1974] M. Demazure, "Désingularisation des variétés de Schubert généralisées”, Ann. Sci. École Norm. Sup. (4) 7 (1974), 53-88. MR 50 \#7174 Zbl 0312.14009

[Edidin and Graham 1997] D. Edidin and W. Graham, "Characteristic classes in the Chow ring", J. Algebraic Geom. 6:3 (1997), 431-443. MR 98m:14007 Zbl 0922.14003

[Edidin and Graham 1998] D. Edidin and W. Graham, "Equivariant intersection theory", Invent. Math. 131:3 (1998), 595-634. MR 99j:14003a Zbl 0940.14003

[Edidin and Graham 2000] D. Edidin and W. Graham, "Riemann-Roch for equivariant Chow groups", Duke Math. J. 102:3 (2000), 567-594. MR 2001f:14018 Zbl 0997.14002

[Ellingsrud and Strømme 1989] G. Ellingsrud and S. A. Strømme, "On the Chow ring of a geometric quotient”, Ann. of Math. (2) 130:1 (1989), 159-187. MR 90h:14019 Zbl 0716.14002 
[Fulton 1984] W. Fulton, Intersection theory, Ergebnisse der Mathematik und ihrer Grenzgebiete (3) 2, Springer, Berlin, 1984. MR 85k:14004 Zbl 0541.14005

[Fulton 1993] W. Fulton, Introduction to toric varieties, Annals of Mathematics Studies 131, Princeton University Press, 1993. MR 94g:14028 Zbl 0813.14039

[Holm and Sjamaar 2008] T. S. Holm and R. Sjamaar, "Torsion and abelianization in equivariant cohomology”, Transform. Groups 13:3-4 (2008), 585-615. MR 2009j:57041 Zbl 1221.55007

[Kahn 1999] B. Kahn, "Motivic cohomology of smooth geometrically cellular varieties", pp. 149-174 in Algebraic K-theory (Seattle, WA, 1997), edited by W. Raskind and C. Weibel, Proc. Sympos. Pure Math. 67, Amer. Math. Soc., Providence, RI, 1999. MR 2001b:14031 Zbl 0954.19004

[Krishna 2009a] A. Krishna, "Equivariant $K$-theory and higher Chow groups of smooth varieties", preprint, 2009. arXiv 0906.3109

[Krishna 2009b] A. Krishna, "Riemann-Roch for equivariant $K$-theory", preprint, 2009. arXiv 0906. 1696

[Krishna and Levine 2008] A. Krishna and M. Levine, "Additive higher Chow groups of schemes", J. Reine Angew. Math. 619 (2008), 75-140. MR 2009d:14005 Zbl 1158.14009

[M. Demazure 1970] A. G. M. Demazure (editor), Schémas en groupes, III, Lecture Notes in Math. 153, Springer, Berlin, 1970. Zbl 0212.52810

[Merkurjev 2005] A. S. Merkurjev, "Equivariant $K$-theory", pp. 925-954 in Handbook of $K$-theory, vol. 2, edited by E. M. Friedlander and D. R. Grayson, Springer, Berlin, 2005. MR 2006m:14011 Zbl 1108.19002

[Mumford et al. 1994] D. Mumford, J. Fogarty, and F. Kirwan, Geometric invariant theory, 3rd ed., Ergebnisse der Mathematik und ihrer Grenzgebiete (2) 34, Springer, Berlin, 1994. MR 95m:14012 Zbl 0797.14004

[Panin 2003] I. Panin, "Oriented cohomology theories of algebraic varieties", K-Theory 30:3 (2003), 265-314. MR 2005f:14043 Zbl 1047.19001

[Sankaran and Uma 2003] P. Sankaran and V. Uma, "Cohomology of toric bundles", Comment. Math. Helv. 78:3 (2003), 540-554. MR 2004f:14076 Zbl 1050.14047

[Springer 1998] T. A. Springer, Linear algebraic groups, 2nd ed., Progress in Mathematics 9, Birkhäuser, Boston, MA, 1998. MR 99h:20075 Zbl 0927.20024

[Sumihiro 1975] H. Sumihiro, "Equivariant completion, II", J. Math. Kyoto Univ. 15:3 (1975), 573-605. MR 52 \#8137 Zbl 0331.14008

[Thomason 1986] R. W. Thomason, "Lefschetz-Riemann-Roch theorem and coherent trace formula", Invent. Math. 85:3 (1986), 515-543. MR 87j:14028 Zbl 0653.14005

[Thomason 1988] R. W. Thomason, "Equivariant algebraic vs. topological $K$-homology AtiyahSegal-style”, Duke Math. J. 56:3 (1988), 589-636. MR 89f:14015 Zbl 0655.55002

[Thomason 1993] R. W. Thomason, "Les $K$-groupes d'un schéma éclaté et une formule d'intersection excédentaire”, Invent. Math. 112:1 (1993), 195-215. MR 93k:19005 Zbl 0816.19004

[Totaro 1999] B. Totaro, "The Chow ring of a classifying space", pp. 249-281 in Algebraic K-theory (Seattle, WA, 1997), edited by W. Raskind and C. Weibel, Proc. Sympos. Pure Math. 67, Amer. Math. Soc., Providence, RI, 1999. MR 2001f:14011 Zbl 0967.14005

[Vezzosi and Vistoli 2003] G. Vezzosi and A. Vistoli, "Higher algebraic $K$-theory for actions of diagonalizable groups", Invent. Math. 153:1 (2003), 1-44. MR 2006c:19003 Zbl 1032.19001

[Vezzosi and Vistoli 2005] G. Vezzosi and A. Vistoli, "Erratum to [Vezzosi and Vistoli 2003]", Invent. Math. 161:1 (2005), 219-224. MR 2178662 Zbl 1032.19001 
[Vistoli 1989] A. Vistoli, "Characteristic classes of principal bundles in algebraic intersection theory", Duke Math. J. 58:2 (1989), 299-315. MR 90k:14052 Zbl 0685.14006

Communicated by Hélène Esnault

Received 2011-11-10 Revised 2012-02-28 Accepted 2012-03-28

amal@math.tifr.res.in School of Mathematics, Tata Institute of Fundamental

Research, 1 Homi Bhabha Road, Mumbai 400005, India 


\section{Algebra \& Number Theory}

msp.org/ant

\section{EDITORS}

MANAGING EDITOR

Bjorn Poonen

Massachusetts Institute of Technology

Cambridge, USA

\author{
EDITORIAL BOARD CHAIR \\ David Eisenbud \\ University of California \\ Berkeley, USA
}

\section{BOARD OF EDITORS}

Georgia Benkart

Dave Benson

Richard E. Borcherds

John H. Coates

J-L. Colliot-Thélène

Brian D. Conrad

Hélène Esnault

Hubert Flenner

Edward Frenkel

Andrew Granville

Joseph Gubeladze

Ehud Hrushovski

Craig Huneke

Mikhail Kapranov

Yujiro Kawamata

János Kollár

Yuri Manin

Barry Mazur

Philippe Michel
University of Wisconsin, Madison, USA

University of Aberdeen, Scotland

University of California, Berkeley, USA

University of Cambridge, UK

CNRS, Université Paris-Sud, France

University of Michigan, USA

Freie Universität Berlin, Germany

Ruhr-Universität, Germany

University of California, Berkeley, USA

Université de Montréal, Canada

San Francisco State University, USA

Hebrew University, Israel

University of Virginia, USA

Yale University, USA

University of Tokyo, Japan

Princeton University, USA

Northwestern University, USA

Harvard University, USA

École Polytechnique Fédérale de Lausanne
Susan Montgomery

Shigefumi Mori

Raman Parimala

Jonathan Pila

Victor Reiner

Karl Rubin

Peter Sarnak

Joseph H. Silverman

Michael Singer

Vasudevan Srinivas

J. Toby Stafford

Bernd Sturmfels

Richard Taylor

Ravi Vakil

Michel van den Bergh

Marie-France Vignéras

Kei-Ichi Watanabe

Efim Zelmanov
University of Southern California, USA

RIMS, Kyoto University, Japan

Emory University, USA

University of Oxford, UK

University of Minnesota, USA

University of California, Irvine, USA

Princeton University, USA

Brown University, USA

North Carolina State University, USA

Tata Inst. of Fund. Research, India

University of Michigan, USA

University of California, Berkeley, USA

Harvard University, USA

Stanford University, USA

Hasselt University, Belgium

Université Paris VII, France

Nihon University, Japan

University of California, San Diego, USA

\section{PRODUCTION}

production@msp.org

Silvio Levy, Scientific Editor

See inside back cover or msp.org/ant for submission instructions.

The subscription price for 2013 is US \$200/year for the electronic version, and \$350/year ( $\$ 40$, if shipping outside the US) for print and electronic. Subscriptions, requests for back issues and changes of subscribers address should be sent to MSP.

Algebra \& Number Theory (ISSN 1944-7833 electronic, 1937-0652 printed) at Mathematical Sciences Publishers, 798 Evans Hall \#3840, c/o University of California, Berkeley, CA 94720-3840 is published continuously online. Periodical rate postage paid at Berkeley, CA 94704, and additional mailing offices.

ANT peer review and production are managed by EditFLOW ${ }^{\circledR}$ from Mathematical Sciences Publishers.

\section{PUBLISHED BY}

mathematical sciences publishers

nonprofit scientific publishing

http://msp.org/

(C) 2013 Mathematical Sciences Publishers 


\section{Algebra \& Number Theory}

$\begin{array}{lll}\text { Volume } 7 & \text { No. } 2 & 2013\end{array}$

The system of representations of the Weil-Deligne group associated to an abelian variety 243 RUTGER NOOT

Fourier-Jacobi coefficients of Eisenstein series on unitary groups

BEI ZHANG

The phase limit set of a variety

MoUnIR NisSE and FRANK SoTTILE

Base change behavior of the relative canonical sheaf related to higher dimensional moduli

Zsolt PATAKFALVI

Two ways to degenerate the Jacobian are the same

JESSE LEO KASS

Arithmetic motivic Poincaré series of toric varieties

Helena Cobo Pablos and Pedro Daniel González Pérez

Maximal ideals and representations of twisted forms of algebras

Michael LAU and ARTURo PiAnZOLA

Higher Chow groups of varieties with group action 